\title{
Complex linear Diophantine fuzzy sets and their cosine similarity measures with applications
}

\author{
Hüseyin Kamacl ${ }^{1}$ \\ Received: 10 April 2020 / Accepted: 19 October 2021 / Published online: 6 December 2021 \\ (c) The Author(s) 2021
}

\begin{abstract}
In this paper, the concept of complex linear Diophantine fuzzy set (CLDFS), which is obtained by integrating the phase term into the structure of the linear Diophantine fuzzy set (LDFS) and thus is an extension of LDFS, is introduced. In other words, the ranges of grades of membership, non-membership, and reference parameters in the structure of LDFS are extended from the interval $[0,1]$ to unit circle in the complex plane. Besides, this set approach is proposed to remove the conditions associated with the grades of complex-valued membership and complex-valued non-membership in the framework of complex intuitionistic fuzzy set (CIFS), complex Pythagorean fuzzy set (CPyFS), and complex q-rung orthopair fuzzy set (Cq-ROFS). It is proved that each of CIFS, CPyFS, and Cq-ROFS is a CLDFS, but not vice versa. In addition, some operations and relations on CLDFSs are derived and their fundamental properties are investigated. The intuitive definitions of cosine similarity measure (CSM) and cosine distance measure (CDM) between two CLDFSs are introduced and their characteristic principles are examined. An approach based on CSM is proposed to tackle medical diagnosis issues and its performance is tested by dealing with numerical examples. Finally, a comparative study of the proposed approach with several existing approaches is created and its advantages are discussed.
\end{abstract}

Keywords Fuzzy set · Complex fuzzy set · Linear Diophantine fuzzy set · Complex linear Diophantine fuzzy set · Cosine similarity measure

\section{Introduction}

In classical set theory, the membership of elements in a set is evaluated in binary terms according to a bivalent condition whether an element belongs to the set. This is insufficient to deal with many issues such as decision-making, medical diagnosis, and pattern recognition in the real world. To eliminate these difficulties, in 1965, Zadeh [1] proposed the fuzzy set (FS), a generalization of the classic set. The fuzzy set theory permits the gradual evaluation of the membership of elements in a set; this is characterized with the aid of a membership function valued in the real unit range $[0$, 1]. Many basic operations and aggregation operators were derived to aggregate two or more fuzzy values. Some information measures were proposed to determine the coefficients of similarity and distance between two objects in a fuzzy set.

Hüseyin Kamac1

huseyin.kamaci@hotmail.com

1 Department of Mathematics, Faculty of Science and Arts, Yozgat Bozok University, 66100 Yozgat, Turkey
The implementations of fuzzy sets in many fields such as decision-making, game theory, and machine learning were investigated. There is still interest in these issues and new concepts based on fuzzy sets are described. In the years following the introduction of the fuzzy set into the literature, many extended versions of the fuzzy set have been developed. The idea of hybridization of fuzzy set with hesitancy or bipolarity was proposed, and thus, the theories of hesitant fuzzy set and bipolar fuzzy set were initiated [2-5]. Atanassov [6] introduced an intuitive notion of fuzzy set, called intuitionistic fuzzy set (IFS), by integrating the non-membership function to the fuzzy set. This paradigm was studied by many authors in various aspects [7-13]. Yager [14] put forward that for some problems in the real-world scene, the sum of membership and non-membership grades in the IFS structure may be greater than 1, and thus described Pythagorean fuzzy set (PyFS) characterized by the membership and nonmembership functions providing the condition: the sum of squares of membership and non-membership grades is less than or equal to 1 . Theoretical directions (like operations, relations, and aggregation operators in [15-17]) and practical 
directions (like information measures in [18-21] and applications in multicriteria decision-making [22-25]) of PyFSs were improved. The PyFS is also known as IFS of type 2 by Atanassov [6]. In 2017, Yager [26] proposed the IFS of type $q$ (where $q \geq 1$ ), called q-rung orthopair fuzzy set (q-ROFS), extending the spaces of FS, IFS, and PyFS. Many researchers contributed to the budding and sprouting of this proposal [2730]. By integrating the indeterminacy degree into these types of fuzzy sets consisting of membership and non-membership degrees, different versions of generalized forms of the fuzzy set were proposed [31-36]. Also, the soft approaches (see: the concept of soft set in [37]) of these uncertainty-based sets were investigated, which are the structures in which objects are classified according to parametric data [38-42]. To achieve calculations in a practical way, their matrix representations were functionally derived [43-46]. Moreover, the applications of these soft set theoretic approaches were widely covered [47-50].

Many researchers also considered, what will be happened when the co-domain of FS was replaced by some set of complex numbers instead of $[0,1]$. This idea was theorized by Ramot [51] in 2002 who described the notion of complex fuzzy set (CFS) as a generalization of FS. The CFS is characterized by a complex-valued function, i.e., $\Delta\left(r_{k}\right) e^{i 2 \pi\left(\xi_{\Delta}\left(r_{k}\right)\right)}$ and satisfied the condition: $0 \leq$ $\Delta\left(r_{k}\right), \xi_{\Delta}\left(r_{k}\right) \leq 1$. The notion related to CFSs was also proposed in [52,53]. As mentioned above, though the CFSs have many advantages, however, there are some situations where it is difficult or impossible to solve the issue by using only complex-valued membership function. To cope with this issue, Alkouri and Salleh [54,55] proposed the novelty of complex intuitionistic fuzzy set (CIFS), where the CIFS is characterized by complex-valued membership and complex-valued non-membership functions i.e., $\left\langle\Delta\left(r_{k}\right) e^{i 2 \pi\left(\xi_{\Delta}\left(r_{k}\right)\right)}, \Phi\left(r_{k}\right) e^{i 2 \pi\left(\xi_{\Phi}\left(r_{k}\right)\right)}\right\rangle$ and satisfied the condition: $0 \leq \Delta\left(r_{k}\right)+\Phi\left(r_{k}\right) \leq 1$ and $0 \leq \xi_{\Delta}\left(r_{k}\right)+\xi_{\Phi}\left(r_{k}\right) \leq 1$. For further work related to CIFSs and their drawbacks, we may refer to [56-59]. The CIFSs having the complex-valued membership and complex-valued non-membership functions are observed to be insufficient to describe some complicated complex intuitive fuzzy information; for instance, if the grades of complex-valued membership and complex-valued non-membership are $0.7 e^{i 2 \pi(0.6)}$ and $0.5 e^{i 2 \pi(0.7)}$, respectively, then the CIFSs cannot correspond this result because of $0.7+0.5=1.2>1$ and $0.6+0.7=1.4>1$. For dealing with such cases, Ullah et al. [60] defined complex Pythagorean fuzzy set (CPyFS), whose constraint is that the sum of the squares of parts of complex-valued membership grade and complex-valued non-membership grade is less than or equal to 1, i.e., $\left\langle\Delta\left(r_{k}\right) e^{i 2 \pi\left(\xi_{\Delta}\left(r_{k}\right)\right)}, \Phi\left(r_{k}\right) e^{i 2 \pi\left(\xi_{\Phi}\left(r_{k}\right)\right)}\right\rangle$ and satisfied the condition: $0 \leq\left(\Delta\left(r_{k}\right)\right)^{2}+\left(\Phi\left(r_{k}\right)\right)^{2} \leq 1$ and $0 \leq\left(\xi_{\Delta}\left(r_{k}\right)\right)^{2}+\left(\xi_{\Phi}\left(r_{k}\right)\right)^{2} \leq 1$. In 2020, Liu et al. [61] extended the concepts of CPyFS and CIFS and thus described complex q-rung orthopair fuzzy set (Cq-ROFS) whose constraint is that $\left\langle\Delta\left(r_{k}\right) e^{i 2 \pi\left(\xi_{\Delta}\left(r_{k}\right)\right)}, \Phi\left(r_{k}\right) e^{i 2 \pi\left(\xi_{\Phi}\left(r_{k}\right)\right)}\right\rangle$ and satisfied the condition: $0 \leq\left(\Delta\left(r_{k}\right)\right)^{q}+\left(\Phi\left(r_{k}\right)\right)^{q} \leq 1$ and $0 \leq\left(\xi_{\Delta}\left(r_{k}\right)\right)^{q}+\left(\xi_{\Phi}\left(r_{k}\right)\right)^{q} \leq 1$. This set can be considered as CIFS of type $q$. These concepts are widely used by the researchers due to the complexity of the problems in real life (see [60,62-64]).

In 2019, Riaz and Hashmi [65] critically examined the restrictions related to membership and non-membership functions in structures of FS, IFS, PyFS, and q-ROFS, and these limitations pointed out numerically. They introduced the linear Diophantine fuzzy set (LDFS) by adding reference parameters to the structure of IFS to eliminate these restrictions. They stated that the concept of LDFS will eradicate the constraints in the existing methodologies of other sets and enable the freely selection of data in practice. Also, they proved that the space of this set is larger than those of the FS, IFS, PyFS, and q-ROFS, using the arbitrary property of the reference parameters (see: Theorem 3.3 and Table 18 in [65]). The grades of membership, non-membership, and reference parameters in the construction of LDFS are realvalued. However, in many scenes of the real life, the grades of membership, non-membership, and reference parameters may appear as complex-valued. To fill this research gap, in this paper, we propose a complex-valued extension of the LDFS by extending each value of the membership, nonmembership, and reference parameters in construction of LDFS from real number to complex number of the unit circle. Thus, we initiate the theory of complex linear Diophantine fuzzy set (CLDFS) in which the degrees of membership, nonmembership, and reference parameters are complex-valued and are represented in polar coordinates. Through this model, it aims to fill this research gap. The contributions of this study are listed as follows.

- The LDFS, which is the generalized form of FS, IFS, PyFS, and q-ROFS, is insufficient to describe it if the grades of membership, non-membership, and reference parameters for the objects of universal set are complexvalued. However, it is inevitable to encounter complex values during the collection of data in many stages of real-life, and it is necessary to deal with them to reach the result. To eradicate such restrictions and difficulties, the concept of CLDFS is proposed. This is one of the remarkable contributions of the CLDFS.

- There are some interdependent conditions for the grades of complex-valued membership and complex-valued non-membership in structures of CIFS, CPyFS, and CqROFS. For example, CIFS (PyFS) cannot describe some situations due to a restriction that the sum of (squares of) complex-valued membership and complex-valued nonmembership grades cannot exceed 1. In some special situations, the complex-valued membership grade and 
complex-valued non-membership grade are equal to the value $e^{i 2 \pi}$, and so, this points a restriction of Cq-ROFS. By aid of reference parameters, CLDFS has the ability to handle such situations.

- As distinct from the structures of CIFS, CPyFS, and CqROFS, the role of complex-valued reference parameters in the structure of CLDFS is crucial. Indeed, the objects (or issues) can be classified differently by changing the physical sense of complex-valued reference parameters.

- The similarity measure and distance measure are important tools for determining the degrees of similarity or dissimilarity between two objects. The measures of similarity and distance between FSs (or IFSs, PyFSs, q-ROFSs, CFSs, CIFSs, CPyFSs, and Cq-ROFSs), as information measure in fuzzy mathematics, have engaged attention from researchers due to their wide applications in various fields. These information measures are very useful in some areas, such as pattern recognition, machine learning, decision-making, and image processing. This paper proposes new methods to measure similarity and distance between the CLDFSs, and gives some examples and comparative matches to show the flexibility and superiority of the proposed methods.

The remainder of this paper is shown as follows. The section "Preliminaries" briefly introduces the fundamental conceptions of FSs, CFSs, IFSs, CIFSs, PyFSs, CPyFSs, qROFSs, Cq-ROFSs, and LDFSs and the characteristic laws for cosine similarity measures. In the section "Complex linear Diophantine fuzzy sets", we describe the construction of CLDFS, and then propose some operations, propositions, and the methods of comparing two complex linear Diophantine fuzzy numbers (CLDFNs). In the section "Cosine similarity measures for complex linear Diophantine fuzzy sets", we propose two new approaches to calculate the coefficients of similarity and dissimilarity between two CLDFSs. In the section "A novel approach based on CSM with applications", we give some practical examples to prove the effectiveness and superiority of the proposed approaches. The section "Comparative study" is devoted to the comparison analysis of the emerging approaches with the preexisting approaches based on IFS, PyFS, q-ROFS, CIFS, and CPyFS. In the section "Comparative study", we present the conclusions.

\section{Preliminaries}

This section is to remind the paradigms of FS, CFS, IFS, CIFS, PyFS, CPyFS, q-ROFS, Cq-ROFS, LDFS, and the procedure of cosine similarity measure.

Definition 2.1 [1] Let $\Re$ be a universal discourse set. Then, a fuzzy set $(\mathrm{FS}) \mathcal{F}$ in the universal discourse set $\Re$ is an object form

$\mathcal{F}=\left\{\left(r_{k},\left\langle\Delta_{\mathcal{F}}\left(r_{k}\right)\right\rangle\right): r_{k} \in \Re\right\}$,

where $\Delta_{\mathcal{F}}\left(r_{k}\right) \in[0,1]$ represents the grade of membership of $r_{k} \in \Re$ into the set $\mathcal{F}$.

Definition 2.2 [51] Let $\Re$ be a universal discourse set. Then, a complex fuzzy set (CFS) $\mathfrak{F}$ in the universal discourse set $\Re$ is an object formpagebreak

$\mathfrak{F}=\left\{\left(r_{k},\left\langle\widetilde{\Delta}_{\mathfrak{F}}\left(r_{k}\right)\right\rangle\right): r_{k} \in \mathfrak{R}\right\}$,

where $\widetilde{\Delta}_{\mathfrak{F}}\left(r_{k}\right)=\Delta_{\mathfrak{F}}\left(r_{k}\right) e^{i \vartheta_{\mathcal{F}}}\left(r_{k}\right)$ (for $\Delta_{\mathfrak{F}}\left(r_{k}\right) \in[0,1]$ and $\left.\vartheta_{\Delta_{\mathfrak{F}}}\left(r_{k}\right) \in[0,2 \pi]\right)$ represents the grade of complex-valued membership of $r_{k} \in \Re$ into the set $\mathcal{C}$.

The notion of complex fuzzy set can be revisited as follows:

$\mathfrak{F}=\left\{\left(r_{k},\left\langle\Delta_{\mathfrak{F}}\left(r_{k}\right) e^{i 2 \pi\left(\xi_{\Delta}\left(r_{k}\right)\right)}\right\rangle\right): r_{k} \in \mathfrak{R}\right\}$

where $\Delta_{\mathfrak{F}}\left(r_{k}\right) e^{i 2 \pi\left(\xi_{\Delta_{\mathfrak{F}}}\left(r_{k}\right)\right)}\left(\right.$ for $\left.\Delta_{\mathfrak{F}}\left(r_{k}\right), \xi_{\Delta_{\mathfrak{F}}}\left(r_{k}\right) \in[0,1]\right)$ represents the grade of complex-valued membership of $r_{k} \in$ $\mathfrak{R}$ into the set $\mathcal{C}$.

Definition 2.3 [6] Let $\Re$ be a universal discourse set. Then, an intuitionistic fuzzy set (IFS) $\mathcal{I}$ in the universal discourse set $\mathfrak{R}$ is given by

$\mathcal{I}=\left\{\left(r_{k},\left\langle\Delta_{\mathcal{I}}\left(r_{k}\right), \Phi_{\mathcal{I}}\left(r_{k}\right)\right\rangle\right): r_{k} \in \Re\right\}$,

where $\Delta_{\mathcal{I}}\left(r_{k}\right), \Phi_{\mathcal{I}}\left(r_{k}\right) \in[0,1]$ represent the grades of membership and non-membership of $r_{k} \in \mathfrak{R}$ into the set $\mathcal{I}$ with the condition $0 \leq \Delta_{\mathcal{I}}\left(r_{k}\right)+\Phi_{\mathcal{I}}\left(r_{k}\right) \leq 1$.

Definition 2.4 [54,55] Let $\Re$ be a universal discourse set. Then, a complex intuitionistic fuzzy set (CIFS) $\mathfrak{I}$ in the universal discourse set $\Re$ is described as

$\mathfrak{I}=\left\{\left(r_{k},\left\langle\Delta_{\mathfrak{I}}\left(r_{k}\right) e^{i 2 \pi\left(\xi_{\Delta \mathfrak{I}}\left(r_{k}\right)\right)}, \Phi_{\mathfrak{I}}\left(r_{k}\right) e^{i 2 \pi\left(\xi_{\Phi_{\mathfrak{I}}}\left(r_{k}\right)\right)}\right\rangle\right): r_{k} \in \mathfrak{R}\right\}$,

where $\Delta_{\mathfrak{I}}\left(r_{k}\right) e^{i 2 \pi\left(\xi_{\Delta_{\mathfrak{I}}}\left(r_{k}\right)\right)}$ and $\Phi_{\mathfrak{I}}\left(r_{k}\right) e^{i 2 \pi\left(\xi_{\Phi_{\mathfrak{I}}}\left(r_{k}\right)\right)}$ represent the grades of complex-valued membership and complexvalued non-membership of $r_{k} \in \mathfrak{R}$ into the set $\mathfrak{I}$ with the conditions $0 \leq \Delta_{\mathfrak{I}}\left(r_{k}\right)+\Phi_{\mathfrak{I}}\left(r_{k}\right) \leq 1$ and $0 \leq \xi_{\Delta \mathfrak{I}}\left(r_{k}\right)+$ $\xi_{\Phi_{\mathfrak{I}}}\left(r_{k}\right) \leq 1$. The set of all CIFSs over the universal discourse $\Re$ is denoted by $C I F S(\Re)$.

Definition 2.5 [14] Let $\Re$ be a universal discourse set. Then, a Pythagorean fuzzy set (PyFS) $\mathcal{P}$ in the universal discourse set $\mathfrak{R}$ is an object form

$\mathcal{P}=\left\{\left(r_{k},\left\langle\Delta_{\mathcal{P}}\left(r_{k}\right), \Phi_{\mathcal{P}}\left(r_{k}\right)\right\rangle\right): r_{k} \in \mathfrak{R}\right\}$

where $\Delta_{\mathcal{P}}\left(r_{k}\right), \Phi_{\mathcal{P}}\left(r_{k}\right) \in[0,1]$ represent the grades of membership and non-membership of $r_{k} \in \mathfrak{R}$ into the set $\mathcal{P}$ with the condition $0 \leq\left(\Delta_{\mathcal{P}}\left(r_{k}\right)\right)^{2}+\left(\Phi_{\mathcal{P}}\left(r_{k}\right)\right)^{2} \leq 1$. 
Definition 2.6 [60] Let $\Re$ be a universal discourse set. Then, a complex Pythagorean fuzzy set (CPyFS) $\mathfrak{P}$ in the universal discourse set $\Re$ is defined as

$\mathfrak{P}=\left\{\left(r_{k},\left\langle\Delta_{\mathfrak{P}}\left(r_{k}\right) e^{i 2 \pi\left(\xi_{\Delta_{\mathfrak{P}}}\left(r_{k}\right)\right)}, \Phi_{\mathfrak{P}}\left(r_{k}\right) e^{i 2 \pi\left(\xi_{\mathfrak{P}}\left(r_{k}\right)\right)}\right\rangle\right): r_{k} \in \mathfrak{R}\right\}$,

where $\Delta_{\mathfrak{P}}\left(r_{k}\right) e^{i 2 \pi\left(\xi_{\mathfrak{P}^{(}\left(r_{k}\right)}\right)}$ and $\Phi_{\mathfrak{P}}\left(r_{k}\right) e^{i 2 \pi\left(\xi_{\Phi_{\mathfrak{P}}\left(r_{k}\right)}\right)}$ represent the grades of complex-valued membership and complexvalued non-membership of $r_{k} \in \mathfrak{R}$ into the set $\mathfrak{P}$ with the conditions $0 \leq\left(\Delta_{\mathfrak{P}}\left(r_{k}\right)\right)^{2}+\left(\Phi_{\mathfrak{P}}\left(r_{k}\right)\right)^{2} \leq 1$ and $0 \leq\left(\xi_{\Delta_{\mathfrak{P}}}\left(r_{k}\right)\right)^{2}+\left(\xi_{\Phi_{\mathfrak{P}}}\left(r_{k}\right)\right)^{2} \leq 1$. The set of all CPyFSs over the universal discourse $\Re$ is denoted by $C P y F S(\Re)$.

Definition 2.7 [26] Let $\Re$ be a universal discourse set. Then, a q-rung orthopair fuzzy set (q-ROFS) $\mathcal{Q}$ in the universal discourse set $\Re$ is an object form

$\mathcal{Q}=\left\{\left(r_{k},\left\langle\Delta_{\mathcal{Q}}\left(r_{k}\right), \Phi_{\amalg}\left(r_{k}\right)\right\rangle\right): r_{k} \in \Re\right\}$,

where $\Delta_{\mathcal{Q}}\left(r_{k}\right), \Phi_{\mathcal{Q}}\left(r_{k}\right) \in[0,1]$ represent the grades of membership and non-membership of $r_{k} \in \Re$ into the set $\mathcal{Q}$ with the condition $0 \leq\left(\Delta_{\mathcal{P}}\left(r_{k}\right)\right)^{q}+\left(\Phi_{\mathcal{P}}\left(r_{k}\right)\right)^{q} \leq 1$.

Definition 2.8 [61] Let $\Re$ be a universal discourse set. Then, a complex q-rung orthopair fuzzy set (Cq-ROFS) $\mathfrak{P}$ in the universal discourse set $\Re$ is given by

$\mathfrak{Q}=\left\{\left(r_{k},\left\langle\Delta_{\mathfrak{Q}}\left(r_{k}\right) e^{i 2 \pi\left(\xi_{\Delta_{\mathfrak{Q}}}\left(r_{k}\right)\right)}, \Phi_{\mathfrak{Q}}\left(r_{k}\right) e^{i 2 \pi\left(\xi_{\Phi_{\mathfrak{Q}}}\left(r_{k}\right)\right)}\right\rangle\right): r_{k} \in \mathfrak{R}\right\}$,

where $\Delta_{\mathfrak{Q}}\left(r_{k}\right) e^{i 2 \pi\left(\xi_{\Delta_{\mathfrak{Q}}\left(r_{k}\right)}\right)}$ and $\Phi_{\mathfrak{Q}}\left(r_{k}\right) e^{i 2 \pi\left(\xi_{\Phi_{\mathfrak{Q}}\left(r_{k}\right)}\right)}$ represent the grades of complex-valued membership and complexvalued non-membership of $r_{k} \in \Re$ into the set $\mathfrak{Q}$ with the conditions $0 \leq\left(\Delta_{\mathfrak{Q}}\left(r_{k}\right)\right)^{q}+\left(\Phi_{\mathfrak{Q}}\left(r_{k}\right)\right)^{q} \leq 1$ and $0 \leq\left(\xi_{\Delta_{\mathfrak{Q}}}\left(r_{k}\right)\right)^{q}+\left(\xi_{\Phi_{\mathfrak{Q}}}\left(r_{k}\right)\right)^{q} \leq 1$. The set of all Cq-ROFSs over the universal discourse $\Re$ is denoted by $C q-R O F S(\Re)$.

In 2019, Riaz and Hashmi [65] defined the linear Diophantine fuzzy sets, which are the generalized forms of the IFSs, PyFSs, and q-ROFSs, by integrating the grades of reference parameters to the grades of membership and non-membership in the constructions of the IFS, PyFS, and q-ROFS.

Definition 2.9 [65] Let $\Re$ be a universal discourse set. Then, a linear Diophantine fuzzy set (LDFS) $\mathcal{L}$ in the universal discourse set $\Re$ is an object form

$\mathcal{L}=\left\{\left(r_{k},\left\langle\Delta_{\mathcal{L}}\left(r_{k}\right), \Phi_{\mathcal{L}}\left(r_{k}\right)\right\rangle,\left\langle\rho_{\mathcal{L}}^{k}, \sigma_{\mathcal{L}}^{k}\right\rangle\right): r_{k} \in \Re\right\}$,

where $\Delta_{\mathcal{L}}\left(r_{k}\right), \Phi_{\mathcal{L}}\left(r_{k}\right), \rho_{\mathcal{L}}^{k}, \sigma_{\mathcal{I}}^{k} \in[0,1]$, respectively, represent the grades of membership, non-membership, and references parameters of $r_{k} \in \Re$ into the set $\mathcal{L}$ with the conditions $0 \leq \rho_{\mathcal{L}}^{k}+\sigma_{\mathcal{L}}^{k} \leq 1$ and $0 \leq \rho_{\mathcal{L}}^{k} \Delta_{\mathcal{L}}\left(r_{k}\right)+\sigma_{\mathcal{L}}^{k} \Phi_{\mathcal{L}}\left(r_{k}\right) \leq 1$.
Cosine similarity measure $[66,67]$ is described as the inner product of two vectors divided by the product of their lengths. The cosine similarity measure is described in the following.

Let $A=\left(a_{1}, a_{2}, \ldots, a_{s}\right)$ and $B=\left(b_{1}, b_{2}, \ldots, b_{s}\right)$ be two vectors of length $s$. Then, the cosine similarity measure between these two vectors is denoted and defined by

$\mathfrak{C}(A, B)=\frac{A B}{\|A\|\|B\|}=\frac{\sum_{k=1}^{s} a_{k} b_{k}}{\sqrt{\sum_{k=1}^{s} a_{k}^{2}} \sqrt{\sum_{k=1}^{s} b_{k}^{2}}}$,

where $A B=\sum_{k=1}^{s} a_{k} b_{k}$ represents the inner product of the vectors $A$ and $B$, and $\|A\|=\sqrt{\sum_{k=1}^{s} a_{k}^{2}},\|B\|=\sqrt{\sum_{k=1}^{s} b_{k}^{2}}$ represent the Euclidean norms of vectors $A$ and $B$, respectively. However, if $a_{k}=b_{k}=0$ for $k=1,2, \ldots, s$, then it is undefined. In such cases, the measure value is assume as zero.

The cosine similarity measure for two vectors satisfies the following properties:

1. $0 \leq \mathfrak{C}(A, B) \leq 1$.

2. $\mathfrak{C}(A, B)=\mathfrak{C}(B, A)$.

3. $A=B \Leftrightarrow \mathfrak{C}(A, B)=1$.

\section{Complex linear Diophantine fuzzy sets}

In this section, we describe the concepts of complex linear Diophantine fuzzy set and complex linear Diophantine fuzzy number, and then investigate their operational laws.

\section{Construction of complex linear Diophantine fuzzy set}

Definition 3.1 Let $\Re$ be a universal discourse set. Then, a complex linear Diophantine fuzzy set (CLDFS) $\mathfrak{L}$ in the universal discourse set $\Re$ is an object form

$$
\begin{aligned}
& \mathfrak{L}=\left\{\left(r_{k},\left\langle\Delta_{\mathfrak{L}}\left(r_{k}\right) e^{i 2 \pi\left(\xi_{\Delta_{\mathfrak{L}}}\left(r_{k}\right)\right)}, \Phi_{\mathfrak{L}}\left(r_{k}\right) e^{i 2 \pi\left(\xi_{\Phi_{\mathfrak{L}}}\left(r_{k}\right)\right)}\right\rangle,\langle\right.\right. \\
& \left.\left.\left.\rho_{\mathfrak{L}}^{k} e^{i 2 \pi\left(\xi_{\rho_{\mathfrak{L}}^{k}}\right)}, \sigma_{\mathfrak{L}}^{k} e^{i 2 \pi\left(\xi_{\sigma_{\mathfrak{L}}^{k}}\right)}\right\rangle\right): r_{k} \in \mathfrak{R}\right\},
\end{aligned}
$$

where $\Delta_{\mathfrak{L}}\left(r_{k}\right) e^{i 2 \pi\left(\xi_{\Delta_{\mathfrak{L}}\left(r_{k}\right)}\right)}, \Phi_{\mathfrak{L}}\left(r_{k}\right) e^{i 2 \pi\left(\xi_{\Phi_{\mathfrak{L}}\left(r_{k}\right)}\right)}, \rho_{\mathfrak{L}}^{k} e^{i 2 \pi\left(\xi_{\rho_{\mathfrak{L}}^{k}}\right)}$, $\sigma_{\mathfrak{L}}^{k} e^{i 2 \pi\left(\xi_{\sigma_{\mathfrak{L}}^{k}}\right)}$, respectively, represent the grades of complexvalued membership, complex-valued non-membership, and complex-valued reference parameters of $r_{k} \in \Re$ into the set $\mathfrak{L}$ with the conditions $0 \leq \rho_{\mathfrak{L}}^{k}+\sigma_{\mathfrak{L}}^{k} \leq 1,0 \leq \rho_{\mathfrak{L}}^{k} \Delta_{\mathfrak{L}}\left(r_{k}\right)+$ $\sigma_{\mathfrak{L}}^{k} \Phi_{\mathfrak{L}}\left(r_{k}\right) \leq 1$ and $0 \leq \xi_{\rho_{\mathfrak{L}}^{k}}+\xi_{\sigma_{\mathfrak{L}}^{k}} \leq 1,0 \leq \xi_{\rho_{\mathfrak{L}}^{k}} \xi_{\Delta_{\mathfrak{L}}}\left(r_{k}\right)+$ $\xi_{\sigma_{\mathfrak{L}}^{k}} \xi_{\Phi_{\mathfrak{L}}}\left(r_{k}\right) \leq 1$. The hesitation part can be evaluated $\varrho_{\mathfrak{L}} \Theta_{\mathfrak{L}}=\left(\varrho_{\mathfrak{L}}^{k} \Theta_{\mathfrak{L}}\left(r_{k}\right)\right) e^{i 2 \pi\left(\xi_{\left(\varrho_{\mathfrak{L}}^{k} \Theta_{\mathfrak{L}}\right)}\left(r_{k}\right)\right)}=\left(1-\left(\rho_{\mathfrak{L}}^{k} \Delta_{\mathfrak{L}}\left(r_{k}\right)+\right.\right.$ $\left.\left.\sigma_{\mathfrak{L}}^{k} \Phi_{\mathfrak{L}}\left(r_{k}\right)\right)\right) e^{i 2 \pi\left(1-\left(\xi_{\rho_{\mathfrak{L}}^{k}} \xi_{\Delta_{\mathfrak{L}}}\left(r_{k}\right)+\xi_{\sigma_{\mathfrak{L}}} \xi_{\Phi_{\mathfrak{L}}}\left(r_{k}\right)\right)\right)}$ where $\varrho_{\mathfrak{L}}$ is the 
reference parameter related to grade of indeterminacy. Any element $\quad\left(\left\langle\Delta_{\mathfrak{L}} e^{i 2 \pi\left(\xi_{\Delta_{\mathfrak{L}}}\right)}, \Phi_{\mathfrak{L}} e^{i 2 \pi\left(\xi_{\Phi_{\mathfrak{L}}}\right)}\right\rangle\right.$, $\left.\left\langle\rho_{\mathfrak{L}} e^{i 2 \pi\left(\xi_{\rho_{\mathfrak{L}}}\right)}, \sigma_{\mathfrak{L}} e^{i 2 \pi\left(\xi_{\sigma_{\mathfrak{L}}}\right)}\right\rangle\right)$ of CLDFS is simply denoted by $\ell=\left(\left\langle\left(\Delta_{\mathfrak{L}}, \xi_{\Delta_{\mathfrak{L}}}\right),\left(\Phi_{\mathfrak{L}}, \xi_{\Phi_{\mathfrak{L}}}\right)\right\rangle,\left\langle\left(\rho_{\mathfrak{L}}, \xi_{\rho_{\mathfrak{L}}}\right),\left(\sigma_{\mathfrak{L}}, \xi_{\sigma_{\mathfrak{L}}}\right)\right\rangle\right)$ and is generally called complex linear Diophantine fuzzy number (CLDFN). The set of all CLDFSs over the universal discourse $\Re$ is denoted by $C L D F S(\Re)$.

Example 3.2 The application range of CLDFS is wide. Pattern recognition, medical diagnosis, and artificial intelligence are just a few of the application areas. Let us talk about their applications in today's technology. The signals are sent to the receiver via conductive or electromagnetic waves which can bring energy into a system by virtue of their electric and magnetic fields and have various features. The depth, amplitude, and distance of signal affect the quality of information or data. In addition to these physical characteristics of signal, electronic noise, which are unwanted signals interfering in the transmission paths to sent signals, and electromagnetic interference, whose sources may be environmental such as electrical storms and solar radiation, are also important for quality transmission of information or data. Also, it is sometimes difficult to express data with real-valued memberships in evaluating the properties of the signals, and in such cases, data can be quantified with complex-valued memberships.

Suppose that five electromagnetic signals $r_{1}, r_{2}, r_{3}, r_{4}, r_{5}$ have been received by a receiver. One may want to determine the best signal having favorable physical properties and low-frequency electronic noise. Considering the reference parameters as "low-frequency electronic noise" and "not lowfrequency electronic noise", we create the following CLDFS:

$$
\mathfrak{L}=\left\{\begin{array}{l}
\left(r_{1},\left\langle 0.6 e^{i 2 \pi(0.7)}, 0.8 e^{i 2 \pi(0.1)}\right\rangle,\right. \\
\left.\left\langle 0.4 e^{i 2 \pi(0.3)}, 0.5 e^{i 2 \pi(0.2)}\right\rangle\right), \\
\left(r_{2},\left\langle 0.5 e^{i 2 \pi(0.5)}, 0.8 e^{i 2 \pi(0.6)}\right\rangle,\right. \\
\left.\left\langle 0.3 e^{i 2 \pi(1)}, 0.7 e^{i 2 \pi(0)}\right\rangle\right), \\
\left(r_{3},\left\langle 0.6 e^{i 2 \pi(0.6)}, 0.7 e^{i 2 \pi(0.3)}\right\rangle,\right. \\
\left.\left\langle 0.8 e^{i 2 \pi(0.3)}, 0.1 e^{i 2 \pi(0.3)}\right\rangle\right), \\
\left(r_{4},\left\langle 0.7 e^{i 2 \pi(0.5)}, 0.7 e^{i 2 \pi(1)}\right\rangle,\right. \\
\left.\left\langle 0.4 e^{i 2 \pi(0.1)}, 0.4 e^{i 2 \pi(0.6)}\right\rangle\right), \\
\left(r_{5},\left\langle 0.6 e^{i 2 \pi(0.4)}, 0.3 e^{i 2 \pi(0.8)}\right\rangle,\right. \\
\left.\left\langle 0.5 e^{i 2 \pi(0.1)}, 0.5 e^{i 2 \pi(0.4)}\right\rangle\right)
\end{array}\right\} .
$$

Tabular form representing this CLDFS $\mathfrak{L}$ is presented in Table 1.

Electromagnetic interference describes the phenomenon caused by allowing conducted and radiated signals to reach destinations where their presence is undesirable. If we consider the reference parameters as "low-frequency electromagnetic interference" and "not low-frequency electromagnetic interference", then for these reference parameters, the CLDF data can take the form as Table 2.
Table 1 CLDFS for reference parameter "electronic noise"

\begin{tabular}{lll}
\hline $\mathfrak{L}$ & $\left\langle\left(\Delta_{\mathfrak{L}}\left(r_{k}\right), \xi_{\Delta_{\mathfrak{L}}}\left(r_{k}\right)\right),\left(\Phi_{\mathfrak{L}}\left(r_{k}\right), \xi_{\Phi_{\mathfrak{L}}}\left(r_{k}\right)\right)\right\rangle$ & $\left\langle\left(\rho_{\mathfrak{L}}^{k}, \xi_{\rho_{\mathfrak{L}}^{k}}\right),\left(\sigma_{\mathfrak{L}}^{k}, \xi_{\sigma_{\mathfrak{L}}^{k}}\right)\right\rangle$ \\
\hline$r_{1}$ & $\langle(0.6,0.7),(0.8,0.1)\rangle$ & $\langle(0.4,0.3),(0.5,0.2)\rangle$ \\
$r_{2}$ & $\langle(0.5,0.5),(0.8,0.6)\rangle$ & $\langle(0.3,1),(0.7,0)\rangle$ \\
$r_{3}$ & $\langle(0.6,0.6),(0.7,0.3)\rangle$ & $\langle(0.8,0.3),(0.1,0.3)\rangle$ \\
$r_{4}$ & $\langle(0.7,0.5),(0.7,1)\rangle$ & $\langle(0.4,0.1),(0.4,0.6)\rangle$ \\
$r_{5}$ & $\langle(0.6,0.4),(0.3,0.8)\rangle$ & $\langle(0.5,0.1),(0.5,0.4)\rangle$ \\
\hline
\end{tabular}

Table 2 CLDFS for reference parameter "electromagnetic interference"

\begin{tabular}{lll}
\hline $\mathfrak{L}$ & $\left\langle\left(\Delta_{\mathfrak{L}}\left(r_{k}\right), \xi_{\Delta_{\mathfrak{L}}}\left(r_{k}\right)\right),\left(\Phi_{\mathfrak{L}}\left(r_{k}\right), \xi_{\Phi_{\mathfrak{L}}}\left(r_{k}\right)\right)\right\rangle$ & $\left\langle\left(\rho_{\mathfrak{L}}^{k}, \xi_{\rho_{\mathfrak{L}}^{k}}\right),\left(\sigma_{\mathfrak{L}}^{k}, \xi_{\sigma_{\mathfrak{L}}^{k}}\right)\right\rangle$ \\
\hline$r_{1}$ & $\langle(0.5,0.8),(0.7,0.6)\rangle$ & $\langle(0.4,0.5),(0.6,0.2)\rangle$ \\
$r_{2}$ & $\langle(0.4,0.3),(0.7,0.3)\rangle$ & $\langle(0.7,0.6),(0.2,0.2)\rangle$ \\
$r_{3}$ & $\langle(0.4,0.4),(0.6,0.5)\rangle$ & $\langle(0.4,0.4),(0.5,0.2)\rangle$ \\
$r_{4}$ & $\langle(1,0.8),(0.5,0.3)\rangle$ & $\langle(0.5,0.3),(0.3,0.7)\rangle$ \\
$r_{5}$ & $\langle(0.9,0.1),(0.4,0.4)\rangle$ & $\langle(0.2,0.3),(0.8,0.5)\rangle$ \\
\hline
\end{tabular}

Proposition 3.3 The CIFN, CPyFN, and Cq-ROFN are also CLDFNs, but may not vice versa.

Proof Let $\ell=\left(\left\langle\left(\Delta_{\mathfrak{L}}, \xi_{\Delta_{\mathfrak{L}}}\right),\left(\Phi_{\mathfrak{L}}, \xi_{\Phi_{\mathfrak{L}}}\right)\right\rangle,\left\langle\left(\rho_{\mathfrak{L}}, \xi_{\rho_{\mathfrak{L}}}\right),\left(\sigma_{\mathfrak{L}}\right.\right.\right.$, $\left.\left.\left.\xi_{\sigma_{\mathfrak{L}}}\right)\right\rangle\right)$ be a CLDFN with the conditions $0 \leq \rho_{\mathfrak{L}}+\sigma_{\mathfrak{L}} \leq 1$, $0 \leq \rho_{\mathfrak{L}} \Delta_{\mathfrak{L}}+\sigma_{\mathfrak{L}} \Phi_{\mathfrak{L}} \leq 1$ and $0 \leq \xi_{\rho_{\mathfrak{L}}}+\xi_{\sigma_{\mathfrak{L}}} \leq 1,0 \leq$ $\xi_{\rho_{\mathfrak{L}}} \xi_{\Delta_{\mathfrak{L}}}+\xi_{\sigma_{\mathfrak{L}}} \xi_{\Phi_{\mathfrak{L}}} \leq 1$ where $\rho_{\mathfrak{L}}, \sigma_{\mathfrak{L}}, \Delta_{\mathfrak{L}}, \Phi_{\mathfrak{L}}, \xi_{\rho_{\mathfrak{L}}}, \xi_{\sigma_{\mathfrak{L}}}$, $\xi_{\Delta_{\mathfrak{L}}}, \xi_{\Phi_{\mathfrak{L}}} \in[0,1]$. It is obvious that considering the arbitrary choice of complex-valued reference parameters, the above inequalities are achieved for every CIFN, CPyFN, and CqROFN. Thus, we have that each of CIFN, CPyFN, and CqROFN is also a CLDFN.

Contrary, an example can be considered. If $\Delta_{\mathfrak{L}}=1$, $\Phi_{\mathfrak{L}}=1, \xi_{\Delta_{\mathfrak{L}}}=1$, and $\xi_{\Phi_{\mathfrak{L}}}=1$, then we have $1^{q}+$ $1^{q}=2 \nless 1$ for any $q \geq 1$. However, for the arbitrary choice of complex-valued reference parameters, we obtain $0 \leq \rho_{\mathfrak{L}} \Delta_{\mathfrak{L}}+\sigma_{\mathfrak{L}} \Phi_{\mathfrak{L}} \leq 1$ and $0 \leq \xi_{\rho_{\mathfrak{L}}} \xi_{\Delta_{\mathfrak{L}}}+\xi_{\sigma_{\mathfrak{L}}} \xi_{\Phi_{\mathfrak{L}}} \leq 1$. Then, we can say that any CLDFN may not be CIFN, CPyFN, and $\mathrm{Cq}-\mathrm{ROFN}$.

In conclusion, CLDFS is a complex-valued extended version of LDFS, and thus, FS, IFS, PyFS, and q-ROFS (see Table 18 in [65]). Also, $C L D F S(\Re) \supseteq C q-R O F S(\Re) \supseteq$ $C P y F S(\Re) \supseteq C I F S(\Re) \supseteq C F S(\Re)$, since the complexvalued reference parameters in the structure of CLDFS can be arbitrarily taken by Proposition 3.3. 
Definition 3.4 A CLDFS in the universal discourse set $\Re$ of the form $\mathfrak{L}^{1}=\left\{\left(r_{k},\left\langle 1 e^{i 2 \pi(1)}, 0 e^{i 2 \pi(0)}\right\rangle,\left\langle 1 e^{i 2 \pi(1)}, 0 e^{i 2 \pi(0)}\right\rangle\right)\right.$ : $\left.r_{k} \in \mathfrak{R}\right\}=\left\{\left(r_{k},\langle 1,0\rangle,\langle 1,0\rangle\right): r_{k} \in \mathfrak{R}\right\}$ is said to be absolute CLDFS and $\mathfrak{L}^{0}=\left\{\left(r_{k},\left\langle 0 e^{i 2 \pi(0)}, 1 e^{i 2 \pi(1)}\right\rangle\right.\right.$, $\left.\left.\left\langle 0 e^{i 2 \pi(0)}, 1 e^{i 2 \pi(1)}\right\rangle\right): r_{k} \in \mathfrak{R}\right\}=\left\{\left(r_{k},\langle 0,1\rangle,\langle 0,1\rangle\right): r_{k} \in\right.$ $\Re$ \} is said to be null or empty CLDFS.

Definition 3.5 Let $\mathfrak{L}_{1}, \mathfrak{L}_{2} \in C L D F S(\Re)$. Then

(a) $\mathfrak{L}_{1}$ is subset of $\mathfrak{L}_{2}$, denoted by $\mathfrak{L}_{1} \subseteq \mathfrak{L}_{2}$, if

$$
\left\{\begin{array}{l}
\Delta_{\mathfrak{L}_{1}}\left(r_{k}\right) \leq \Delta_{\mathfrak{L}_{2}}\left(r_{k}\right), \\
\xi_{\Delta_{\mathfrak{L}_{1}}}\left(r_{k}\right) \leq \xi_{\Delta_{\mathfrak{L}_{2}}}\left(r_{k}\right), \\
\Phi_{\mathfrak{L}_{1}}\left(r_{k}\right) \geq \Phi_{\mathfrak{L}_{2}}\left(r_{k}\right), \\
\xi_{\Phi_{\mathfrak{L}_{1}}}\left(r_{k}\right) \geq \xi_{\Phi_{\mathfrak{L}_{2}}}\left(r_{k}\right), \\
\rho_{\mathfrak{L}_{1}} \leq \rho_{\mathfrak{L}_{2}}, \xi_{\rho_{\mathfrak{L}_{1}}} \leq \xi_{\rho_{\mathfrak{L}_{2}}}, \\
\sigma_{\mathfrak{L}_{1}} \geq \sigma_{\mathfrak{L}_{2}}, \xi_{\sigma_{\mathfrak{L}_{1}}} \geq \xi_{\sigma_{\mathfrak{S}_{2}}}
\end{array}\right\}_{(k=1,2, \ldots, s)} ;
$$

(b) $\mathfrak{L}_{1}$ is equal of $\mathfrak{L}_{2}$, denoted by $\mathfrak{L}_{1}=\mathfrak{L}_{2}$, if

$$
\left\{\begin{array}{l}
\Delta_{\mathfrak{L}_{1}}\left(r_{k}\right)=\Delta_{\mathfrak{L}_{2}}\left(r_{k}\right), \\
\xi_{\Delta_{\mathfrak{L}_{1}}}\left(r_{k}\right)=\xi_{\Delta_{\mathfrak{S}_{2}}}\left(r_{k}\right), \\
\Phi_{\mathfrak{L}_{1}}\left(r_{k}\right)=\Phi_{\mathfrak{L}_{2}}\left(r_{k}\right), \\
\xi_{\Phi_{\mathfrak{L}_{1}}}\left(r_{k}\right)=\xi_{\Phi_{\mathfrak{L}_{2}}}\left(r_{k}\right), \\
\rho_{\mathfrak{L}_{1}}=\rho_{\mathfrak{L}_{2}}, \xi_{\rho_{\mathfrak{S}_{1}}}=\xi_{\rho_{\mathfrak{L}_{2}}}, \\
\sigma_{\mathfrak{L}_{1}}=\sigma_{\mathfrak{L}_{2}}, \xi_{\sigma_{\mathfrak{L}_{1}}}=\xi_{\sigma_{\mathfrak{L}_{2}}}
\end{array}\right\}_{(k=1,2, \ldots, s)}
$$

\section{Some basic operations on complex linear Diophantine fuzzy sets}

Definition 3.6 Let $\mathfrak{L}_{j}=\left\{\left(r_{k},\left\langle\Delta_{\mathfrak{L}_{j}}\left(r_{k}\right) e^{i 2 \pi\left(\xi_{\Delta_{\mathfrak{L}_{j}}}\left(r_{k}\right)\right)}\right.\right.\right.$, $\left.\left.\Phi_{\mathfrak{L}_{j}}\left(r_{k}\right) e^{i 2 \pi\left(\xi_{\Phi_{\mathfrak{L}_{j}}}\left(r_{k}\right)\right)}\right\rangle,\left\langle\rho_{\mathfrak{L}_{j}}^{k} e^{i 2 \pi\left(\xi_{\rho_{\mathfrak{L}_{j}}^{k}}\right)}, \sigma_{\mathfrak{L}_{j}}^{k} e^{i 2 \pi\left(\xi_{\sigma_{\mathfrak{L}_{j}}^{k}}\right)}\right\rangle\right): r_{k}$ $\in \Re\} \in C L D F S(\Re)$ for $j=1,2, \ldots, n$. Then, we have the following operations:

(a) (Complement of CLDFS) $\mathfrak{L}_{j}^{c}=\left\{\left(r_{k},\left\langle\Phi_{\mathfrak{L}_{j}}\left(r_{k}\right)\right.\right.\right.$

$$
\begin{aligned}
& \left.e^{i 2 \pi\left(\xi_{\Phi_{\mathcal{L}_{j}}}\left(r_{k}\right)\right)}, \Delta \mathcal{L}_{j}\left(r_{k}\right) e^{i 2 \pi\left(\xi_{\mathcal{L}_{j}}\left(r_{k}\right)\right)}\right\rangle,\left\langle\sigma_{\mathfrak{L}_{j}}^{k} e^{i 2 \pi\left(\xi_{\sigma_{\mathfrak{L}_{j}}^{k}}\right)},\right. \\
& \left.\left.\left.\rho_{\mathfrak{L}_{j}}^{k} e^{i 2 \pi\left(\xi_{\rho_{\mathfrak{L}_{j}}^{k}}^{k}\right)}\right\rangle\right): r_{k} \in \Re\right\} .
\end{aligned}
$$

(b) (Intersection of CLDFSs) $\bigcap_{j=1}^{n} \mathfrak{L}_{j}=\left\{\left(r_{k},\left\langle\Delta \widetilde{\cap}_{\mathfrak{L}_{j}}\left(r_{k}\right)\right.\right.\right.$

$$
\begin{aligned}
& \left.e^{i 2 \pi\left(\xi_{\Delta \tilde{\cap}_{j}}\left(r_{k}\right)\right)}, \Phi_{\widetilde{\cap}_{\mathfrak{L}_{j}}}\left(r_{k}\right) e^{i 2 \pi\left(\xi_{\tilde{ก} \Phi_{\mathfrak{L}_{j}}}\left(r_{k}\right)\right)}\right\rangle, \\
& \left.\left.\left\langle\rho_{\tilde{\cap} \mathfrak{L}_{j}}^{k} e^{i 2 \pi\left(\xi_{\rho_{\tilde{\cap}}^{k} \mathfrak{L}_{j}}\right)}, \sigma_{\tilde{\cap} \mathfrak{L}_{j}}^{k} e^{i 2 \pi\left(\xi_{\sigma_{\tilde{n}}^{k} \mathfrak{L}_{j}}\right)}\right\rangle\right): r_{k} \in \Re\right\} \text { where }
\end{aligned}
$$

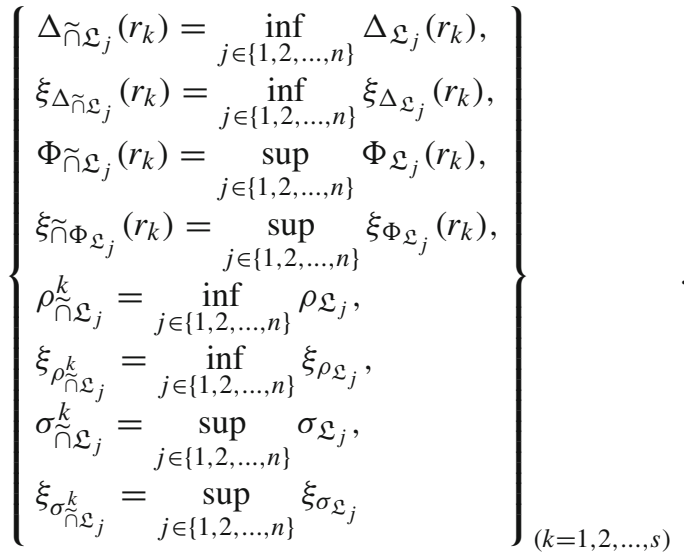

(c) (Union of CLDFSs) $\bigcup_{j=1}^{n} \mathfrak{L}_{j}=\left\{\left(r_{k},\left\langle\Delta_{\widetilde{\cup} \mathfrak{L}_{j}}\left(r_{k}\right)\right.\right.\right.$

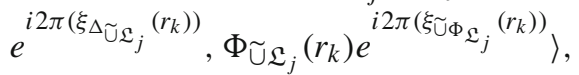

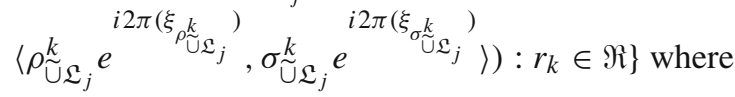

$$
\left\{\begin{array}{l}
\Delta_{\widetilde{\cup} \mathfrak{L}_{j}}\left(r_{k}\right)=\sup _{j \in\{1,2, \ldots, n\}} \Delta_{\mathfrak{L}_{j}}\left(r_{k}\right), \\
\xi_{\Delta \widetilde{\cup} \mathfrak{L}_{j}}\left(r_{k}\right)=\sup _{j \in\{1,2, \ldots, n\}} \xi_{\Delta_{\mathfrak{L}_{j}}}\left(r_{k}\right), \\
\Phi_{\widetilde{\cup} \mathfrak{L}_{j}}\left(r_{k}\right)=\inf _{j \in\{1,2, \ldots, n\}} \Phi_{\mathfrak{L}_{j}}\left(r_{k}\right), \\
\xi_{\widetilde{\cup} \Phi_{\mathfrak{L}_{j}}}\left(r_{k}\right)=\inf _{j \in\{1,2, \ldots, n\}} \xi_{\Phi_{\mathfrak{L}_{j}}}\left(r_{k}\right), \\
\rho_{\widetilde{\cup} \mathfrak{L}_{j}}^{k}=\sup _{j \in\{1,2, \ldots, n\}} \rho_{\mathfrak{L}_{j}}, \\
\xi_{\rho_{\tilde{\cup} \mathfrak{L}_{j}}^{k}}=\sup _{j \in\{1,2, \ldots, n\}} \xi_{\rho_{\mathfrak{L}_{j}},}, \\
\sigma_{\widetilde{\cup} \mathfrak{L}_{j}}^{k}=\inf _{j \in\{1,2, \ldots, n\}} \sigma_{\mathfrak{L}_{j}}, \\
\xi_{\sigma_{\tilde{\cup} \mathfrak{L}_{j}}^{k}}=\inf _{j \in\{1,2, \ldots, n\}} \xi_{\sigma_{\mathfrak{L}_{j}}}
\end{array}\right\}_{(k=1,2, \ldots, s)}
$$

Example 3.7 Let us consider the following CLDFSs $\mathfrak{L}_{1}, \mathfrak{L}_{2}$ and $\mathfrak{L}_{3}$ in the universal set $\Re=\left\{r_{1}, r_{2}\right\}$ :

$$
\begin{aligned}
& \mathfrak{L}_{1}=\left\{\left(r_{1},\left\langle 0.5 e^{i 2 \pi(0.9)}, 0.4 e^{i 2 \pi(0.6)}\right\rangle,\right.\right. \\
& \left.\left\langle 0.7 e^{i 2 \pi(0.6)}, 0.2 e^{i 2 \pi(0.3)}\right\rangle\right), \\
& \left(r_{2},\left\langle 0.5 e^{i 2 \pi(0.7)}, 0.8 e^{i 2 \pi(0.7)}\right\rangle,\right. \\
& \left.\left.\left\langle 0.5 e^{i 2 \pi(0.5)}, 0.4 e^{i 2 \pi(0.4)}\right\rangle\right)\right\}, \\
& \mathfrak{L}_{2}=\left\{\left(r_{1},\left\langle 0.9 e^{i 2 \pi(0.9)}, 0.4 e^{i 2 \pi(0.7)}\right\rangle,\right.\right. \\
& \left.\left\langle 0.2 e^{i 2 \pi(0.4)}, 0.7 e^{i 2 \pi(0.4)}\right\rangle\right), \\
& \left(r_{2},\left\langle 0.3 e^{i 2 \pi(0.8)}, 0.9 e^{i 2 \pi(0.6)}\right\rangle,\right. \\
& \left.\left.\left\langle 0.5 e^{i 2 \pi(0.2)}, 0.5 e^{i 2 \pi(0.3)}\right\rangle\right)\right\}, \\
& \mathfrak{L}_{3}=\left\{\left(r_{1},\left\langle 0.3 e^{i 2 \pi(0.6)}, 0.4 e^{i 2 \pi(0.8)}\right\rangle,\right.\right. \\
& \left.\left\langle 0.7 e^{i 2 \pi(0.4)}, 0.1 e^{i 2 \pi(0.5)}\right\rangle\right), \\
& \left(r_{2},\left\langle 0.3 e^{i 2 \pi(0.9)}, 0.6 e^{i 2 \pi(0.9)}\right\rangle,\right.
\end{aligned}
$$




$$
\left.\left.\left\langle 0.4 e^{i 2 \pi(0.3)}, 0.4 e^{i 2 \pi(0.6)}\right\rangle\right)\right\}
$$

Then, we obtain

i. $\mathfrak{L}_{1}^{c}=\left\{\left(r_{1},\left\langle 0.4 e^{i 2 \pi(0.6)}, 0.5 e^{i 2 \pi(0.9)}\right\rangle,\left\langle 0.2 e^{i 2 \pi(0.3)}\right.\right.\right.$, $\left.\left.0.7 e^{i 2 \pi(0.6)}\right\rangle\right),\left(r_{2},\left\langle 0.8 e^{i 2 \pi(0.7)}, 0.5 e^{i 2 \pi(0.7)}\right\rangle\right.$, $\left.\left.\left\langle 0.4 e^{i 2 \pi(0.4)}, 0.5 e^{i 2 \pi(0.5)}\right\rangle\right)\right\}$.

ii. $\bigcap_{j=1}^{3} \mathfrak{L}_{j}=\left\{\left(r_{1},\left\langle 0.3 e^{i 2 \pi(0.6)}, 0.4 e^{i 2 \pi(0.6)}\right\rangle,\left\langle 0.2 e^{i 2 \pi(0.4)}\right.\right.\right.$, $\left.\left.0.1 e^{i 2 \pi(0.3)}\right\rangle\right),\left(r_{2},\left\langle 0.3 e^{i 2 \pi(0.7)}, 0.6 e^{i 2 \pi(0.6)}\right\rangle\right.$, $\left.\left.\left\langle 0.4 e^{i 2 \pi(0.2)}, 0.4 e^{i 2 \pi(0.3)}\right\rangle\right)\right\}$.

iii. $\bigcup_{j=1}^{3} \mathfrak{L}_{j}=\left\{\left(r_{1},\left\langle 0.9 e^{i 2 \pi(0.9)}, 0.4 e^{i 2 \pi(0.8)}\right\rangle,\left\langle 0.7 e^{i 2 \pi(0.6)}\right.\right.\right.$, $\left.\left.0.7 e^{i 2 \pi(0.5)}\right\rangle\right),\left(r_{2},\left\langle 0.5 e^{i 2 \pi(0.9)}, 0.9 e^{i 2 \pi(0.9)}\right\rangle\right.$, $\left.\left.\left\langle 0.5 e^{i 2 \pi(0.5)}, 0.5 e^{i 2 \pi(0.6)}\right\rangle\right)\right\}$.

Proposition 3.8 Let $\mathfrak{L}_{j} \in \operatorname{CLDFS(\Re )}(j=1,2, \ldots, n)$, and then, $\mathfrak{L}_{j}^{c}, \bigcap_{j=1}^{n} \mathfrak{L}_{j}$ and $\bigcup_{j=1}^{n} \mathfrak{L}_{j}$ are also CLDFSs in the universal discourse $\Re$.

Proof Let $\mathfrak{L}_{j} \in C \operatorname{CDFS}(\Re)(j=1,2, \ldots, n)$. Then, we prove that $\bigcup_{j=1}^{n} \mathfrak{L}_{j}$ is also a CLDFS. Others can be similarly proved.

To prove that $\bigcup_{j=1}^{n} \mathfrak{L}_{j}$ is a CLDFS, the following conditions should be provided: $0 \leq \rho_{\widetilde{\cup} \mathfrak{L}_{j}}^{k}+\sigma_{\widetilde{\cup} \mathfrak{L}_{j}}^{k} \leq 1,0 \leq$ $\rho_{\widetilde{\cup} \mathfrak{L}_{j}}^{k} \Delta_{\widetilde{\cup} \mathfrak{L}_{j}}\left(r_{k}\right)+\sigma_{\widetilde{\cup} \mathfrak{L}_{j}}^{k} \Phi_{\widetilde{\cup} \mathfrak{L}_{j}}\left(r_{k}\right) \leq 1$ and $0 \leq \xi_{\rho_{\widetilde{\cup} \mathfrak{L}_{j}}^{k}}+$ $\xi_{\sigma_{\widetilde{\cup} \mathfrak{L}_{j}}^{k}} \leq 1,0 \leq \xi_{\rho_{\widetilde{\cup} \mathfrak{L}_{j}}^{k}} \xi_{\Delta \widetilde{\cup} \mathfrak{L}_{j}}\left(r_{k}\right)+\xi_{\sigma_{\widetilde{\cup} \mathfrak{L}_{j}}^{k}} \xi_{\Phi_{\mathfrak{L}}}\left(r_{k}\right) \leq 1$ for

\section{Operational laws of complex linear Diophantine fuzzy numbers}

Definition 3.10 The basic operational laws for two CLDFNs $\ell_{1}=\left(\left\langle\left(\Delta_{\mathfrak{L}}^{1}, \xi_{\Delta_{\mathfrak{L}}^{1}}\right),\left(\Phi_{\mathfrak{L}}^{1}, \xi_{\Phi_{\mathfrak{L}}^{1}}\right),\right\rangle,\left\langle\left(\rho_{\mathfrak{L}}^{1}, \xi_{\rho_{\mathfrak{L}}^{1}}\right),\left(\sigma_{\mathfrak{L}}^{1}, \xi_{\sigma_{\mathfrak{L}}^{1}}\right)\right\rangle\right)$ and $\ell_{2}=\left(\left\langle\left(\Delta_{\mathfrak{L}}^{2}, \xi_{\Delta_{\mathfrak{L}}^{2}}\right),\left(\Phi_{\mathfrak{L}}^{2}, \xi_{\Phi_{\mathfrak{L}}^{2}}\right),\right\rangle,\left\langle\left(\rho_{\mathfrak{L}}^{2}, \xi_{\rho_{\mathfrak{L}}^{2}}\right),\left(\sigma_{\mathfrak{L}}^{2}, \xi_{\sigma_{\mathfrak{L}}^{2}}\right)\right\rangle\right)$ are described as follows:

(a) $\ell_{1} \oplus \ell_{2}=\left(\begin{array}{l}\left.\left(\Delta_{\mathfrak{L}}^{1}+\Delta_{\mathfrak{L}}^{2}-\Delta_{\mathfrak{L}}^{1} \Delta_{\mathfrak{L}}^{2}, \xi_{\Delta_{\mathfrak{L}}^{1}}+\xi_{\Delta_{\mathfrak{L}}^{2}}-\xi_{\Delta_{\mathfrak{L}}^{1}} \xi_{\Delta_{\mathfrak{L}}^{2}}\right),\left(\Phi_{\mathfrak{L}}^{1} \Phi_{\mathfrak{L}}^{2}, \xi_{\Phi_{\mathfrak{L}}^{1}} \xi_{\Phi_{\mathfrak{L}}^{2}}\right)\right\rangle, \\ \left.\left(\rho_{\mathfrak{L}}^{1}+\rho_{\mathfrak{L}}^{2}-\rho_{\mathfrak{L}}^{1} \rho_{\mathfrak{L}}^{2}, \xi_{\rho_{\mathfrak{L}}^{1}}+\xi_{\rho_{\mathfrak{L}}^{2}}-\xi_{\rho_{\mathfrak{L}}} \xi_{\rho_{\mathfrak{L}}^{2}}^{2}\right),\left(\sigma_{\mathfrak{L}}^{1} \sigma_{\mathfrak{L}}^{2}, \xi_{\sigma_{\mathfrak{L}}^{1}} \xi_{\sigma_{\mathfrak{L}}^{2}}\right)\right)^{2}\end{array}\right)$.

(b) $\ell_{1} \otimes \ell_{2}=\left(\begin{array}{l}\left.\left(\Delta_{\mathfrak{L}}^{1} \Delta_{\mathfrak{L}}^{2}, \xi_{\Delta_{\mathfrak{L}}^{1}} \xi_{\Delta_{\mathfrak{L}}^{2}}\right),\left(\Phi_{\mathfrak{L}}^{1}+\Phi_{\mathfrak{L}}^{2}-\Phi_{\mathfrak{L}}^{1} \Phi_{\mathfrak{L}}^{2}, \xi_{\Phi_{\mathfrak{L}}^{1}}+\xi_{\Phi_{\mathfrak{L}}^{2}}-\xi_{\Phi_{\mathfrak{L}}^{1}} \xi_{\Phi_{\mathfrak{L}}^{2}}\right)\right\rangle, \\ \left.\left(\rho_{\mathfrak{L}}^{1} \rho_{\mathfrak{L}}^{2}, \xi_{\rho_{\mathfrak{L}}^{1}} \xi_{\rho_{\mathfrak{L}}^{2}}\right),\left(\sigma_{\mathfrak{L}}^{1}+\sigma_{\mathfrak{L}}^{2}-\sigma_{\mathfrak{L}}^{1} \sigma_{\mathfrak{L}}^{2}, \xi_{\sigma_{\mathfrak{L}}^{1}}+\xi_{\sigma_{\mathfrak{L}}^{2}}-\xi_{\sigma_{\mathfrak{L}}^{1}} \xi_{\sigma_{\mathfrak{L}}^{2}}\right)\right)^{2}\end{array}\right)$.

(c) $\lambda \ell_{1}=\left(\begin{array}{l}\left.\left(1-\left(1-\Delta_{\mathfrak{L}}^{1}\right)^{\lambda}, 1-\left(1-\xi_{\Delta_{\mathfrak{L}}^{1}}\right)^{\lambda}\right),\left(\left(\Phi_{\mathfrak{L}}^{1}\right)^{\lambda},\left(\xi_{\Phi_{\mathfrak{L}}^{1}}\right)^{\lambda}\right)\right\rangle, \\ \left.\left(1-\left(1-\rho_{\mathfrak{L}}^{1}\right)^{\lambda}, 1-\left(1-\xi_{\rho_{\mathfrak{L}}^{1}}\right)^{\lambda}\right),\left(\left(\sigma_{\mathfrak{L}}^{1}\right)^{\lambda},\left(\xi_{\sigma_{\mathfrak{L}}^{1}}\right)^{\lambda}\right)\right\rangle\end{array}\right)$, 
(d) $\quad \ell_{1}^{\lambda}=\left(\begin{array}{l}\left.\left(\left(\Delta_{\mathfrak{L}}^{1}\right)^{\lambda},\left(\xi_{\Delta_{\mathfrak{L}}^{1}}\right)^{\lambda}\right),\left(1-\left(1-\Phi_{\mathfrak{L}}^{1}\right)^{\lambda}, 1-\left(1-\xi_{\Phi_{\mathfrak{L}}^{1}}\right)^{\lambda}\right)\right\rangle, \\ \left.\left(\left(\rho_{\mathfrak{L}}^{1}\right)^{\lambda},\left(\xi_{\rho_{\mathfrak{L}}^{1}}\right)^{\lambda}\right),\left(1-\left(1-\sigma_{\mathfrak{L}}^{1}\right)^{\lambda}, 1-\left(1-\xi_{\sigma_{\mathfrak{L}}^{1}}\right)^{\lambda}\right)\right\rangle\end{array}\right)$

where $\lambda$ is a positive real number.

Example 3.11 We consider the following two CLDFNs: $\ell_{1}=$ $(\langle(0.7,0.6),(0.8,1)\rangle,\langle(0.1,0.5),(0.5,0.4)\rangle) \quad$ and $\ell_{2}=(\langle(0.4,0.5),(0.8,0.6)\rangle,\langle(0.2,0.5),(0.6,0.4)\rangle)$, and also $\lambda=3$. Then, we obtain

i. $\ell_{1} \oplus \ell_{2}=(\langle(0.82,0.8),(0.64,0.6)\rangle,\langle(0.28,0.75)$, $(0.3,0.16)\rangle)$.

ii. $\ell_{1} \otimes \ell_{2}=(\langle(0.28,0.3),(0.96,1)\rangle,\langle(0.02,0.25)$, $(0.8,0.64)\rangle)$.

iii. $3 \ell_{1}=(\langle(0.973,0.936),(0.512,1)\rangle,\langle(0.271,0.875)$, $(0.125,0.064)\rangle)$.

iv. $\ell_{1}^{3}=(\langle(0.343,0.216),(0.992,1)\rangle,\langle(0.001,0.125)$, $(0.875,0.784)\rangle)$.

Proposition 3.12 If $\ell_{1}$ and $\ell_{2}$ be two CLDFNs and $\lambda>0$, then $\ell_{1} \oplus \ell_{2}, \ell_{1} \otimes \ell_{2}, \lambda \ell_{1}$ and $\ell_{1}^{\lambda}$ are also CLDFNs.

Proof $\ell_{1}=\left(\left\langle\left(\Delta_{\mathfrak{L}}^{1}, \xi_{\Delta_{\mathfrak{L}}^{1}}\right),\left(\Phi_{\mathfrak{L}}^{1}, \xi_{\Phi_{\mathfrak{L}}^{1}}\right),\right\rangle,\left\langle\left(\rho_{\mathfrak{L}}^{1}, \xi_{\rho_{\mathfrak{L}}^{1}}\right),\left(\sigma_{\mathfrak{L}}^{1}\right.\right.\right.$, $\left.\left.\left.\xi_{\sigma_{\mathfrak{L}}^{1}}\right)\right\rangle\right)$ and $\ell_{2}=\left(\left\langle\left(\Delta_{\mathfrak{L}}^{2}, \xi_{\Delta_{\mathfrak{L}}^{2}}\right),\left(\Phi_{\mathfrak{L}}^{2}, \xi_{\Phi_{\mathfrak{L}}^{2}}\right),\right\rangle,\left\langle\left(\rho_{\mathfrak{L}}^{2}, \xi_{\rho_{\mathfrak{L}}^{2}}\right),\left(\sigma_{\mathfrak{L}}^{2}\right.\right.\right.$, $\left.\left.\left.\xi_{\sigma_{\mathfrak{L}}^{2}}\right)\right\rangle\right)$ be two CLDFNs, such that $0 \leq \rho_{\mathfrak{L}}^{t}+\sigma_{\mathfrak{L}}^{t} \leq 1$, $0 \leq \rho_{\mathfrak{L}}^{t} \Delta_{\mathfrak{L}}^{t}+\sigma_{\mathfrak{L}}^{t} \Phi_{\mathfrak{L}}^{t} \leq 1$ and $0 \leq \xi_{\rho_{\mathfrak{L}}^{t}}+\xi_{\sigma_{\mathfrak{L}}^{t}} \leq 1$, $0 \leq \xi_{\rho_{\mathfrak{L}}^{t}} \xi_{\Delta_{\mathcal{L}}^{t}}+\xi_{\sigma_{\mathcal{L}}^{t}} \xi_{\Phi_{\mathcal{L}}^{t}} \leq 1$ for $t=1$, 2. Assume that $\ell_{3}=\ell_{1} \oplus \ell_{2}^{2}$ where
On the other hand, we obtain $\rho_{\mathfrak{\Omega}}^{3}+\sigma_{\mathfrak{\Omega}}^{3} \geq 0$, since $\rho_{\mathfrak{L}}^{1}+\rho_{\mathfrak{L}}^{2} \geq \rho_{\mathfrak{L}}^{1} \rho_{\mathfrak{L}}^{2}$ for $\rho_{\mathfrak{L}}^{1}, \rho_{\mathfrak{L}}^{2} \in[0,1]$. Also, we have $0 \leq$ $\Delta_{\mathfrak{L}}^{3} \leq 1$ and $0 \leq \Phi_{\mathfrak{L}}^{3} \leq 1$ for $\Delta_{\mathfrak{L}}^{1}, \Delta_{\mathfrak{L}}^{2}, \Phi_{\mathfrak{L}}^{1}, \Phi_{\mathfrak{L}}^{2} \in[0,1]$. Consequently, we obtain that $0 \leq \rho_{\mathfrak{L}}^{3} \Delta_{\mathfrak{L}}^{3}+\sigma_{\mathfrak{L}}^{3} \Phi_{\mathfrak{L}}^{3} \leq 1$. Likewise, we can demonstrate that $0 \leq \xi_{\rho_{\mathfrak{L}}^{3}} \xi_{\Delta_{\mathcal{L}}^{3}}+\xi_{\rho_{\mathfrak{L}}^{3}} \xi_{\Phi_{\mathcal{L}}^{3}} \leq 1$. Thus, we get $\ell_{1} \oplus \ell_{2}$ is a CLDFN. Similarly, it can be proved that $\ell_{1} \otimes \ell_{2}, \lambda \ell_{1}$, and $\ell_{1}^{\lambda}$ are also CLDFNs.

Proposition 3.13 Let $\ell_{1}, \ell_{2}$, and $\ell_{3}$ be the CLDFNs and $\lambda>$ 0 . Then, we have

(i) $\ell_{1} \oplus \ell_{2}=\ell_{2} \oplus \ell_{1}$.

(ii) $\ell_{1} \otimes \ell_{2}=\ell_{2} \otimes \ell_{1}$

(iii) $\left(\ell_{1} \oplus \ell_{2}\right) \oplus \ell_{3}=\ell_{1} \oplus\left(\ell_{2} \oplus \ell_{3}\right)$.

(iv) $\left(\ell_{1} \otimes \ell_{2}\right) \otimes \ell_{3}=\ell_{1} \otimes\left(\ell_{2} \otimes \ell_{3}\right)$.

Proof See Appendix.

Proposition 3.14 Let $\ell_{1}, \ell_{2}$, and $\ell_{3}$ be the CLDFNs and $\lambda, \lambda_{1}, \lambda_{2}>0$. Then, we have

(i) $\lambda\left(\ell_{1} \oplus \ell_{2}\right)=\lambda \ell_{1} \oplus \lambda \ell_{2}$.

(ii) $\left(\ell_{1} \otimes \ell_{2}\right)^{\lambda}=\ell_{1}^{\lambda} \otimes \ell_{2}^{\lambda}$.

(iii) $\left(\lambda_{1}+\lambda_{2}\right) \ell_{1}=\lambda_{1} \ell_{1} \oplus \lambda_{2} \ell_{1}$.

(iv) $\ell_{1}^{\lambda_{1}+\lambda_{2}}=\ell_{1}^{\lambda_{1}} \otimes \ell_{1}^{\lambda_{2}}$.

Proof See Appendix.

The operations $\oplus$ and $\otimes$ proposed in Definition 3.10 can be generalized for a collection of CLDFNs as follows.

$\left\{\begin{array}{l}\Delta_{\mathfrak{L}}^{3}=\Delta_{\mathfrak{L}}^{1}+\Delta_{\mathfrak{L}}^{2}-\Delta_{\mathfrak{L}}^{1} \Delta_{\mathfrak{L}}^{2}, \xi_{\Delta_{\mathfrak{L}}^{3}}=\xi_{\Delta_{\mathfrak{L}}^{1}}+\xi_{\Delta_{\mathfrak{L}}^{2}}-\xi_{\Delta_{\mathfrak{L}}^{1}} \xi_{\Delta_{\mathfrak{L}}^{2}}, \Phi_{\mathfrak{L}}^{3}=\Phi_{\mathfrak{L}}^{1} \Phi_{\mathfrak{L}}^{2}, \xi_{\Phi_{\mathfrak{L}}^{3}}=\xi_{\Phi_{\mathfrak{L}}^{1}} \xi_{\Phi_{\mathfrak{L}}^{2}}, \\ \rho_{\mathfrak{L}}^{3}=\rho_{\mathfrak{L}}^{1}+\rho_{\mathfrak{L}}^{2}-\rho_{\mathfrak{L}}^{1} \rho_{\mathfrak{L}}^{2}, \xi_{\rho_{\mathfrak{L}}^{3}}=\xi_{\rho_{\mathfrak{L}}^{1}}+\xi_{\rho_{\mathfrak{L}}^{2}}-\xi_{\rho_{\mathfrak{L}}^{1}} \xi_{\rho_{\mathfrak{L}}^{2}}, \sigma_{\mathfrak{L}}^{3}=\sigma_{\mathfrak{L}}^{1} \sigma_{\mathfrak{L}}^{2}, \xi_{\sigma_{\mathfrak{L}}^{3}}=\xi_{\sigma_{\mathfrak{L}}^{1}} \xi_{\sigma_{\mathfrak{L}}^{2}} .\end{array}\right\}$

We have $0 \leq \rho_{\mathfrak{L}}^{t} \leq 1-\sigma_{\mathfrak{L}}^{t}$, since $0 \leq \rho_{\mathfrak{L}}^{t}+\sigma_{\mathfrak{L}}^{t} \leq 1$ for $t=1,2$. Then, we obtain

$$
\begin{aligned}
\rho_{\mathfrak{L}}^{3}+\sigma_{\mathfrak{L}}^{3}= & \rho_{\mathfrak{L}}^{1}+\rho_{\mathfrak{L}}^{2}-\rho_{\mathfrak{L}}^{1} \rho_{\mathfrak{L}}^{2}+\sigma_{\mathfrak{L}}^{1} \sigma_{\mathfrak{L}}^{2} \\
\leq & 1-\sigma_{\mathfrak{L}}^{1}+1-\sigma_{\mathfrak{L}}^{2}-\left(1-\sigma_{\mathfrak{L}}^{1}\right) \\
& \left(1-\sigma_{\mathfrak{L}}^{2}\right)+\sigma_{\mathfrak{L}}^{1} \sigma_{\mathfrak{L}}^{2} \\
= & 1-\sigma_{\mathfrak{L}}^{1}+1-\sigma_{\mathfrak{L}}^{2}-1 \\
& +\sigma_{\mathfrak{L}}^{1}+\sigma_{\mathfrak{L}}^{2}-\sigma_{\mathfrak{L}}^{1} \sigma_{\mathfrak{L}}^{2}+\sigma_{\mathfrak{L}}^{1} \sigma_{\mathfrak{L}}^{2} \\
= & 1 .
\end{aligned}
$$

Theorem 3.15 Let $\quad \ell_{j} \quad=\quad\left(\left\langle\left(\Delta_{\mathfrak{L}}^{j}, \xi_{\Delta_{\mathfrak{L}}^{j}}\right)\right.\right.$, $\left.\left.\left(\Phi_{\mathfrak{L}}^{j}, \xi_{\Phi_{\mathfrak{L}}^{j}}\right),\right\rangle,\left\langle\left(\rho_{\mathfrak{L}}^{j}, \xi_{\rho_{\mathfrak{L}}^{j}}\right),\left(\sigma_{\mathfrak{L}}^{j}, \xi_{\sigma_{\mathfrak{S}}^{j}}\right)\right\rangle\right)(j=1,2, \ldots, n)$ be a collection of CLDFNs. Then, we have

(a)

$$
\bigoplus_{j=1}^{n} \ell_{j}=\left(\begin{array}{l}
\left\langle\left(1-\prod_{j=1}^{n}\left(1-\Delta_{\mathfrak{L}}^{j}\right), 1-\prod_{j=1}^{n}\left(1-\xi_{\Delta_{\mathfrak{L}}^{j}}\right)\right),\right. \\
\left.\left(\prod_{j=1}^{n} \Phi_{\mathfrak{L}}^{j}, \prod_{j=1}^{n} \xi_{\Phi_{\mathfrak{L}}^{j}}\right)\right\rangle, \\
\left\langle\left(1-\prod_{j=1}^{n}\left(1-\rho_{\mathfrak{L}}^{j}\right), 1-\prod_{j=1}^{n}\left(1-\xi_{\rho_{\mathfrak{L}}^{j}}\right)\right),\right. \\
\left.\left(\prod_{j=1}^{n} \sigma_{\mathfrak{L}}^{j}, \prod_{j=1}^{n} \xi_{\sigma_{\mathfrak{L}}^{j}}\right)\right\rangle
\end{array}\right) .
$$


(b)

$$
\bigotimes_{j=1}^{n} \ell_{j}=\left(\begin{array}{l}
\left\langle\left(\prod_{j=1}^{n} \Delta_{\mathfrak{L}}^{j}, \prod_{j=1}^{n} \xi_{\Delta_{\mathfrak{L}}^{j}}\right),\right. \\
\left.\left(1-\prod_{j=1}^{n}\left(1-\Phi_{\mathfrak{L}}^{j}\right), 1-\prod_{j=1}^{n}\left(1-\xi_{\Phi_{\mathfrak{L}}^{j}}\right)\right)\right\rangle, \\
\left\langle\left(\prod_{j=1}^{n} \rho_{\mathfrak{L}}^{j}, \prod_{j=1}^{n} \xi_{\rho_{\mathfrak{L}}^{j}}\right),\right. \\
\left.\left(1-\prod_{j=1}^{n}\left(1-\sigma_{\mathfrak{L}}^{j}\right), 1-\prod_{j=1}^{n}\left(1-\xi_{\sigma_{\mathfrak{L}}^{j}}\right)\right)\right\rangle
\end{array}\right)
$$

Proof The proofs can be easily seen using mathematical induction.

Now, some concepts are introduced to compare the two CLDFNs and thus determine their priority.

Definition 3.16 Let $\ell=\left(\left\langle\left(\Delta_{\mathfrak{L}}, \xi_{\Delta_{\mathfrak{L}}}\right),\left(\Phi_{\mathfrak{L}}, \xi_{\Phi_{\mathfrak{L}}}\right),\right\rangle\right.$, $\left.\left\langle\left(\rho_{\mathfrak{L}}, \xi_{\rho_{\mathfrak{L}}}\right),\left(\sigma_{\mathfrak{L}}, \xi_{\sigma_{\mathfrak{L}}}\right)\right\rangle\right)$ be a CLDFN.

(a) The score function for $\ell$ is denoted and defined by

$$
\begin{aligned}
\operatorname{Scr}(\ell)= & \frac{1}{4}\left(\left(\Delta_{\mathfrak{L}}-\Phi_{\mathfrak{L}}\right)+\left(\xi_{\Delta_{\mathfrak{L}}}-\xi_{\Phi_{\mathfrak{L}}}\right)+\left(\rho_{\mathfrak{L}}-\sigma_{\mathfrak{L}}\right)\right. \\
& \left.+\left(\xi_{\rho_{\mathfrak{L}}}-\xi_{\sigma_{\mathfrak{L}}}\right)\right), \quad \operatorname{Scr}(\ell) \in[-1,1] .
\end{aligned}
$$

(ii) The accuracy function for $\ell$ is denoted and defined by

$$
\begin{aligned}
\operatorname{Acr}(\ell)= & \frac{1}{4}\left(\left(\frac{\Delta_{\mathfrak{L}}+\Phi_{\mathfrak{L}}}{2}\right)+\left(\frac{\xi_{\Delta_{\mathfrak{L}}}+\xi_{\Phi_{\mathfrak{L}}}}{2}\right)+\left(\rho_{\mathfrak{L}}\right.\right. \\
& \left.\left.+\sigma_{\mathfrak{L}}\right)+\left(\xi_{\rho_{\mathfrak{L}}}+\xi_{\sigma_{\mathfrak{L}}}\right)\right), \quad \operatorname{Acr}(\ell) \in[0,1] .
\end{aligned}
$$

We propose the following comparison strategy to sort two CLDFNs $\ell_{1}$ and $\ell_{2}$.

1. If $\operatorname{Scr}\left(\ell_{1}\right)>\operatorname{Scr}\left(\ell_{2}\right)$, then $\ell_{1}>\ell_{2}$.

2. If $\operatorname{Scr}\left(\ell_{1}\right)<\operatorname{Scr}\left(\ell_{2}\right)$, then $\ell_{1}<\ell_{2}$.

3. If $\operatorname{Scr}\left(\ell_{1}\right)=\operatorname{Scr}\left(\ell_{2}\right)$, then i. if $\operatorname{Acr}\left(\ell_{1}\right)>\operatorname{Acr}\left(\ell_{2}\right)$, then $\ell_{1}>\ell_{2}$,

ii. if $\operatorname{Acr}\left(\ell_{1}\right)<\operatorname{Acr}\left(\ell_{2}\right)$, then $\ell_{1}<\ell_{2}$,

iii. if $\operatorname{Acr}\left(\ell_{1}\right)=\operatorname{Acr}\left(\ell_{2}\right)$, then $\ell_{1}=\ell_{2}$.

Example 3.17 Consider the CLDFNs $\ell_{1}$ and $\ell_{2}$ given in Example 3.11. Then, we have $\operatorname{Scr}\left(\ell_{1}\right)=\operatorname{Scr}\left(\ell_{2}\right)=-0.2$. Using the accuracy function for $\ell_{1}$ and $\ell_{2}$, we obtain $\ell_{1}>\ell_{2}$, since $\operatorname{Acr}\left(\ell_{1}\right)=0.7625>0.7125=\operatorname{Acr}\left(\ell_{2}\right)$.

\section{Cosine similarity measures for complex linear Diophantine fuzzy sets}

In this section, a cosine similarity measure and a weighted cosine similarity measure between CLDFs are proposed based on the concept of the cosine similarity coefficient.

Cosine similarity measure for the FSs is nothing but the cosine of the angle between the vector representations of the two FSs. Suppose that $\mathcal{F}_{1}=\left(\Delta_{\mathcal{F}_{1}}\left(r_{1}\right), \Delta_{\mathcal{F}_{1}}\left(r_{2}\right), \ldots, \Delta_{\mathcal{F}_{1}}\left(r_{s}\right)\right)$ and $\mathcal{F}_{2}=\left(\Delta_{\mathcal{F}_{2}}\left(r_{1}\right), \Delta_{\mathcal{F}_{2}}\left(r_{2}\right), \ldots, \Delta_{\mathcal{F}_{2}}\left(r_{s}\right)\right)$ are the vector representations of two fuzzy sets in the universal set $\Re$. A cosine similarity measure based on Bhattacharya's distance [66,67] between $\mathcal{F}_{1}$ and $\mathcal{F}_{2}$ is described as follows:

$$
\mathfrak{C}_{\text {sim }}\left(\mathcal{F}_{1}, \mathcal{F}_{2}\right)=\frac{\sum_{k=1}^{s} \Delta_{\mathcal{F}_{1}}\left(r_{k}\right) \Delta_{\mathcal{F}_{2}}\left(r_{k}\right)}{\sqrt{\sum_{k=1}^{s}\left(\Delta_{\mathcal{F}_{1}}\left(r_{k}\right)\right)^{2}} \sqrt{\sum_{k=1}^{s}\left(\Delta_{\mathcal{F}_{2}}\left(r_{k}\right)\right)^{2}}} .
$$

Adapting from this, a cosine similarity measure and a weighted cosine similarity measure for CLDFSs can be described in an analogous manner to the cosine similarity measure based on Bhattacharya's distance [66,67].

Suppose that there are two CLDFSs $\mathfrak{L}_{1}$ and $\mathfrak{L}_{2}$ in the universal set $\Re$. Based on the extension of the cosine measure for fuzzy sets, a cosine similarity measure (CSM) between CLDFs $\mathfrak{L}_{1}$ and $\mathfrak{L}_{2}$ is denoted and defined as

$$
\mathfrak{C}_{\text {sim }}\left(\mathfrak{L}_{1}, \mathfrak{L}_{2}\right)=
$$

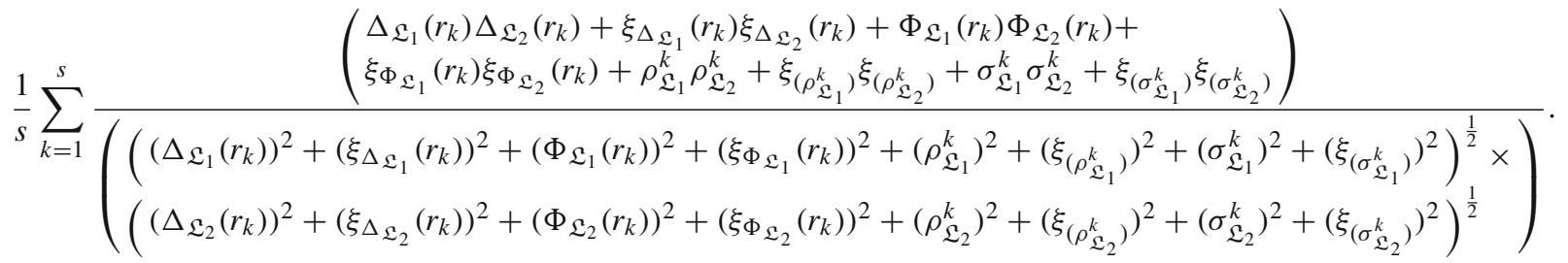

The CSM between CLDFs $\mathfrak{L}_{1}$ and $\mathfrak{L}_{2}$ also satisfies the following properties: 
1. $0 \leq \mathfrak{C}_{\text {sim }}\left(\mathfrak{L}_{1}, \mathfrak{L}_{2}\right) \leq 1$.

2. $\mathfrak{C}_{\text {sim }}\left(\mathfrak{L}_{1}, \mathfrak{L}_{2}\right)=\mathfrak{C}_{\text {sim }}\left(\mathfrak{L}_{2}, \mathfrak{L}_{1}\right)$.

3. $\mathfrak{L}_{1}=\mathfrak{L}_{2} \Leftrightarrow \mathfrak{C}_{\text {sim }}\left(\mathfrak{L}_{1}, \mathfrak{L}_{2}\right)=1$.

Proof See Appendix.

By considering the weighted vector $\varpi$, a weighted cosine similarity measure (WCSM) between CLDFs $\mathfrak{L}_{1}$ and $\mathfrak{L}_{2}$ is denoted and defined as

$\mathfrak{C}_{\text {sim }}^{\varpi}\left(\mathfrak{L}_{1}, \mathfrak{L}_{2}\right)$ $\left.\left.\left.0.2 e^{i 2 \pi(0.3)}\right\rangle\right)\right\}$

i. By (4.2), the CSM between CLDFSs $\mathfrak{L}_{1}$ and $\mathfrak{L}_{2}$ is obtained as $\mathfrak{C}_{\text {sim }}\left(\mathfrak{L}_{1}, \mathfrak{L}_{2}\right)=\frac{1}{2} \times\left(\frac{1.68}{2.2236}+\frac{2.34}{2.34}\right)=$ 0.8877 .

The above CLDFSs $\mathfrak{L}_{1}$ and $\mathfrak{L}_{2}$ consist of two complex linear Diophantine fuzzy elements. Furthermore, $\left(r_{2},\left\langle 0.4 e^{i 2 \pi(0.3)}, 0.9 e^{i 2 \pi(0.9)}\right\rangle,\left\langle 0.5 e^{i 2 \pi(0.3)}, 0.2 e^{i 2 \pi(0.3)}\right\rangle\right) \in$ $\mathfrak{L}_{1} \cap \mathfrak{L}_{2}$. Therefore, we assert that $0.5 \leq \mathfrak{C}_{\text {sim }}\left(\mathfrak{L}_{1}, \mathfrak{L}_{2}\right)$.

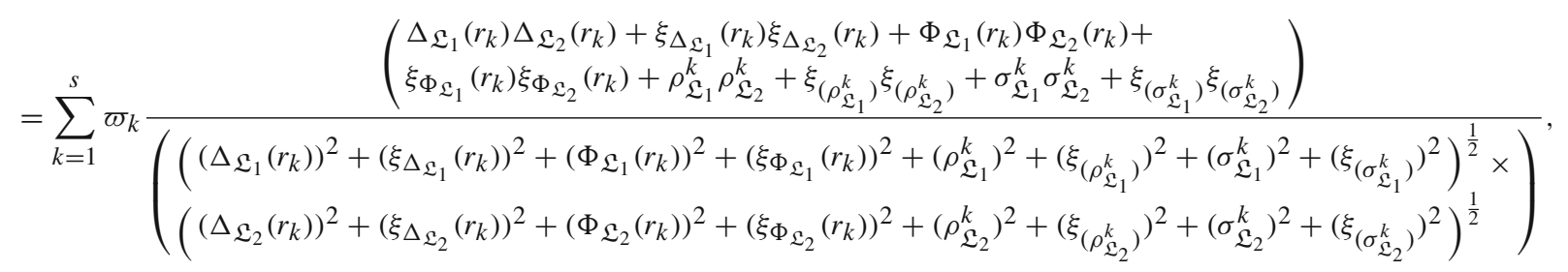

where $\varpi=\left(\varpi_{1}, \varpi_{2}, \ldots, \varpi_{s}\right)^{T}$ is weight vector, such that $\varpi_{k} \in[0,1](k=1,2, \ldots, s)$ and $\sum_{k=1}^{s} \varpi_{k}=1$.

The WCSM between CLDFs $\mathfrak{L}_{1}$ and $\mathfrak{L}_{2}$ also satisfies the following properties:

1. $0 \leq \mathfrak{C}_{\text {sim }}^{\sigma}\left(\mathfrak{L}_{1}, \mathfrak{L}_{2}\right) \leq 1$.

2. $\mathfrak{C}_{\text {sim }}^{\Phi}\left(\mathfrak{L}_{1}, \mathfrak{L}_{2}\right)=\mathfrak{C}_{\text {sim }}^{\pi}\left(\mathfrak{L}_{1}, \mathfrak{L}_{2}\right)$.

3. $\mathfrak{C}_{\text {sim }}^{\text {का }}\left(\mathfrak{L}_{1}, \mathfrak{L}_{2}\right)=1 \Leftrightarrow \mathfrak{L}_{1}=\mathfrak{L}_{2}$.

Proof It can be shown similar to the previous proof.

Note 1: If it is taken $\varpi_{k}=\frac{1}{s}$ for all $k=1,2, \ldots, s$, then WCSM is reduced to CSM, i.e., $\mathfrak{C}_{\text {sim }}^{\sigma}\left(\mathfrak{L}_{1}, \mathfrak{L}_{2}\right)=$ $\mathfrak{C}_{\text {sim }}\left(\mathfrak{L}_{1}, \mathfrak{L}_{2}\right)$.

The cosine distance measure (CDM) and weighted cosine distance measure (WCDM) for CLDFs are denoted and defined as

$\mathfrak{C}_{\text {dis }}\left(\mathfrak{L}_{1}, \mathfrak{L}_{2}\right)=\arccos \left(\mathfrak{C}_{\operatorname{sim}}\left(\mathfrak{L}_{1}, \mathfrak{L}_{2}\right)\right)$,

$\mathfrak{C}_{d i s}^{\varpi}\left(\mathfrak{L}_{1}, \mathfrak{L}_{2}\right)=\arccos \left(\mathfrak{C}_{\text {sim }}^{\varpi}\left(\mathfrak{L}_{1}, \mathfrak{L}_{2}\right)\right)$.

Example 4.1 Let $\Re=\left\{r_{1}, r_{2}\right\}$ be a universal set. Assume that two CLDFSs in $\Re$ are

$$
\begin{aligned}
\mathfrak{L}_{1}= & \left\{\left(r_{1},\left\langle 0.7 e^{i 2 \pi(0.4)}, 0.5 e^{i 2 \pi(0.6)}\right\rangle,\left\langle 0.4 e^{i 2 \pi(0.7)},\right.\right.\right. \\
& \left.\left.0.3 e^{i 2 \pi(0.1)}\right\rangle\right), \\
& \left(r_{2},\left\langle 0.4 e^{i 2 \pi(0.3)}, 0.9 e^{i 2 \pi(0.9)}\right\rangle,\left\langle 0.5 e^{i 2 \pi(0.3)},\right.\right. \\
& \left.\left.\left.0.2 e^{i 2 \pi(0.3)}\right\rangle\right)\right\}, \\
\mathfrak{L}_{2}= & \left\{\left(r_{1},\left\langle 0.2 e^{i 2 \pi(0.5)}, 0.9 e^{i 2 \pi(0.5)}\right\rangle,\left\langle 0.3 e^{i 2 \pi(0.4)},\right.\right.\right. \\
& \left.\left.0.5 e^{i 2 \pi(0.4)}\right\rangle\right), \\
& \left(r_{2},\left\langle 0.4 e^{i 2 \pi(0.3)}, 0.9 e^{i 2 \pi(0.9)}\right\rangle,\left\langle 0.5 e^{i 2 \pi(0.3)},\right.\right.
\end{aligned}
$$

In addition, $\mathfrak{C}_{\text {sim }}\left(\mathfrak{L}_{1}, \mathfrak{L}_{2}\right)<1$ since $\mathfrak{L}_{1} \neq \mathfrak{L}_{2}$. Therefore, we say that $0.5 \leq \mathfrak{C}_{\text {sim }}^{\sigma}\left(\mathfrak{L}_{1}, \mathfrak{L}_{2}\right)<1$. This supports $\mathfrak{C}_{\text {sim }}\left(\mathfrak{L}_{1}, \mathfrak{L}_{2}\right)=0.8877$. Especially, if the weight vector is $\varpi=(0.5,0.5)^{T}$, then the weighted cosine similarity measure is calculated as $\mathfrak{C}_{\text {sim }}^{\sigma}\left(\mathfrak{L}_{1}, \mathfrak{L}_{2}\right)=0.8877$. Hence, the two results coincide.

ii. If we take the weight vector as $\varpi=(0.8,0.2)^{T}$, then we have $\mathfrak{C}_{\text {sim }}^{\varpi}\left(\mathfrak{L}_{1}, \mathfrak{L}_{2}\right)=0.8 \times 0.7755+0.2=0.8204$.

\section{A novel approach based on CSM with applications}

In this section, we present a novel approach based on (weighted) CSM of CLDFSs in line with the aim of creating the most diagnostically accurate method evaluating the medical diagnostic arguments. We support this approach by coping with different numerical problems whose data are collected under CLDFSs environment.

\section{Mathematical modeling}

In this part, we elaborate an algorithm based on (weighted) CSM in handling medical diagnosis issues under the CLDF environment. 


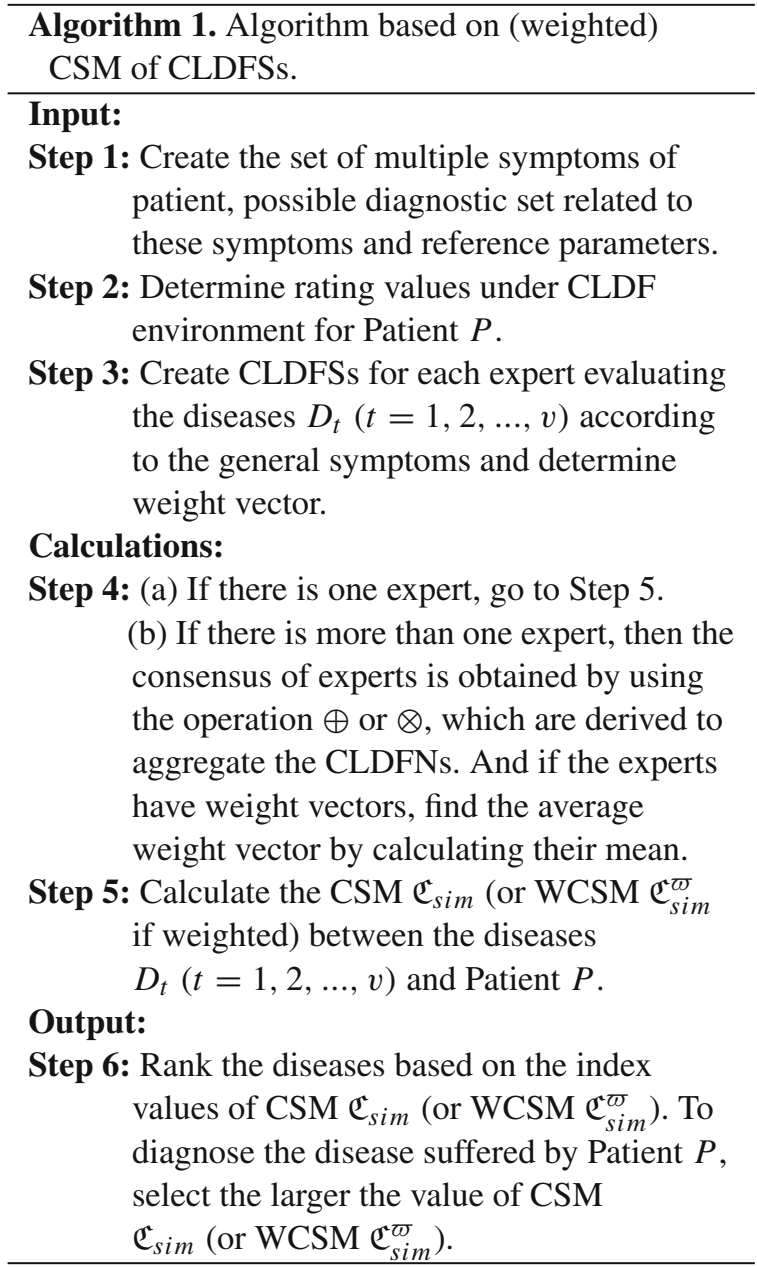

Note 2: Depending on the input data in the medical diagnosis, CDM $\mathfrak{C}_{d i s}$ (or WCDM in $\mathfrak{C}_{d i s}^{\varpi}$ ) can be calculated instead of CSM $\mathfrak{C}_{\text {sim }}$ (or WCSM $\mathfrak{C}_{\text {sim }}^{\varpi}$ ) in Step 5.

To visualize the flow of the algorithm's steps, the flowchart diagram of proposed algorithm is presented in Fig. 1.

\section{Illustrative examples}

In this part, we deal with the fictitious medical diagnosis problems to illustrate the performance of the proposed algorithm.

Example 5.1 To illustrate the application of the proposed cosine measures for CLDFSs to medical diagnosis, we consider the medical diagnosis problem discussed in [12].

Many of the diseases show different symptoms. Medical diagnosis is related to the patient's symptoms to diagnose what type of disease the patient has suffered. The multiple symptoms of patient can be clustered as a collection of symptoms, and different diseases can form a set of diagnostics. However, the frequency of recurrence of these symptoms is also a parameter that gives clues about the disease. For instance, the frequency of recurrence of a cough leads to premonitions about the disease.

Let us consider a set of symptoms

$$
\begin{aligned}
S & =\left\{s_{1}=\text { Cough }, s_{2}=\text { Chest Pain },\right. \\
s_{3} & =\text { Stomach Pain, } \\
s_{4} & \left.=\text { Temperature, } s_{5}=\text { Headache }\right\}
\end{aligned}
$$

and a diagnostic set

$$
\begin{aligned}
D & =\left\{D_{1}=\text { Malaria, } D_{2}=\text { Typhoid },\right. \\
D_{3} & =\text { Viral Fever }, \\
D_{4} & \left.=\text { Chest Problem, } D_{5}=\text { Stomach Problem }\right\}
\end{aligned}
$$

Also, we consider reference parameters "frequently recurrent" and "not frequently recurrent (or rarely recurrent)". Suppose all symptoms and references parameters of a patient can be represented by the CLDFS (data obtained through a survey of doctors) in Table 3.

The symptoms of each disease $D_{t}(t=1,2,3,4,5)$ can also be viewed as CLDFSs (data collected by a medical expert) in Table 4.

Our aim is to diagnose the disease suffered by Patient $P$ by making a similarity test with the classified diseases. Applying the formula in Eq. (4.2), we can obtain the following results:

$$
\begin{aligned}
& \mathfrak{C}_{\text {sim }}\left(P, D_{1}\right)=0.8213, \mathfrak{C}_{\text {sim }}\left(P, D_{2}\right)=0.7636 \\
& \mathfrak{C}_{\text {sim }}\left(P, D_{3}\right)=0.7995, \\
& \mathfrak{C}_{\text {sim }}\left(P, D_{4}\right)=0.9866, \mathfrak{C}_{\text {sim }}\left(P, D_{5}\right)=0.7716
\end{aligned}
$$

Thus, we can assign the patient to the diagnosis $D_{4}$ (Chest Problem) according to the medical diagnosis principle.

If there are weights, then we apply the formula in Eq. (4.3) and get the results as in Table 5 .

The portrayal of the weighted cosine similarity measures given in Table 5 is displayed in Fig. 2.

Now, let us assume that the number of experts in the above example (Example 5.1) is more than one. In such problems, we can use the formulas presented in Theorem 3.15 to aggregate the expert evaluations. (For example, let the CLDFNs of first, second, and third experts be $\ell_{1}^{1}, \ell_{1}^{2}$ and $\ell_{1}^{3}$, respectively, and then, we aggregate these CLDFNs as $\ell_{1}=\ell_{1}^{1} \oplus \ell_{1}^{2} \oplus \ell_{1}^{3}$ or $\ell_{1}=\ell_{1}^{1} \otimes \ell_{1}^{2} \otimes \ell_{1}^{3}$.) Also, the arithmetic average of the weights specified by each expert is calculated, and new weight vector is a series of these values. (For example, the weight vectors specified by the first, second, and third experts are $\varpi^{1}=\left(\varpi_{1}^{1}, \varpi_{2}^{1}, \ldots, \varpi_{s}^{1}\right)^{T}, \varpi^{2}=\left(\varpi_{1}^{2}, \varpi_{2}^{2}, \ldots, \varpi_{s}^{2}\right)^{T}$, and $\varpi^{3}=\left(\varpi_{1}^{2}, \varpi_{2}^{2}, \ldots, \varpi_{s}^{2}\right)^{T}$, respectively, so the new weight vector is obtained as $\varpi=\frac{1}{3} \sum_{j=1}^{3} \varpi^{j}$.) 
Fig. 1 Flowchart diagram of proposed algorithm

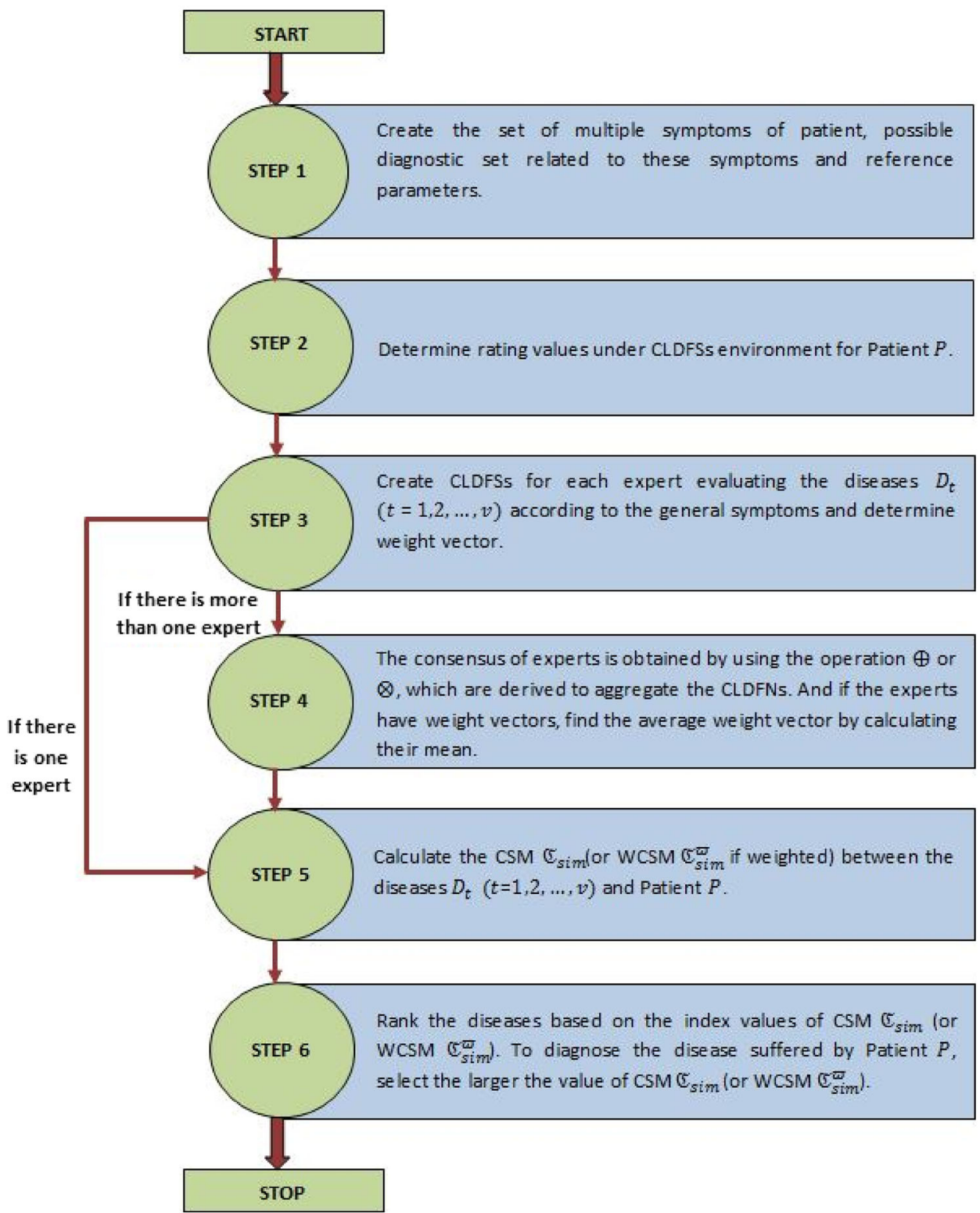

Example 5.2 Let us consider a diagnostic set $D^{*}=\left\{D_{1}^{*}=\right.$ Malaria, $D_{2}^{*}=$ Typhoid, $D_{3}^{*}=$ Viral Fever $\}$, and a set of symptoms and $S^{*}=\left\{s_{1}^{*}=\right.$ Cough, $s_{2}^{*}=$ Temperature, $s_{3}^{*}=$ Headache $\}$. Also, we consider reference parameters "frequently recurrent" and "not frequently recurrent (or rarely recurrent)". Assume all symptoms and reference parameters of a patient can be represented by the CLDFS (data obtained through a survey of doctors) in Table 6.

There are three medical experts in this evaluation process and they evaluate each diagnosis $D_{t}^{*}(t=1,2,3)$ with all the symptoms, which are represented by the CLDFNs, and the results are given in Tables 7, 8, and 9.

If the CLDFSs in Tables 7, 8, and 9 are aggregated by employing the operation $\oplus$, then Table 10 is created.
Assume that the weighted vectors of first, second, and third experts are $\varpi^{1}=(0.5,0.1,0.4)^{T}, \varpi^{2}=(0.4,0.2,0.4)^{T}$ and $\varpi^{3}=(0.3,0.3,0.4)^{T}$. Then, we find that the (average) weight vector is $\varpi=(0.4,0.2,0.4)^{T}$.

Using the formula in Eq. (4.3), we can calculate the weighted cosine similarity measure between $D_{t}^{*}(t=1,2,3)$ and $P^{*}$ as follows:

$$
\begin{array}{r}
\mathfrak{C}_{\text {sim }}^{\Phi}\left(P^{*}, D_{1}^{*}\right)=0.8025, \mathfrak{C}_{\text {sim }}^{\Phi}\left(P^{*}, D_{2}^{*}\right)=0.8392 \\
\text { and } \mathfrak{C}_{\text {sim }}^{\sigma}\left(P^{*}, D_{3}^{*}\right)=0.882 .
\end{array}
$$

Hence, we can assign the patient to the diagnosis $D_{3}$ (Viral Fever) according to the medical diagnosis principle. 
Table 3 CLDFS for patient $P$

\begin{tabular}{clll}
\hline & & $\left\langle\left(\Delta_{\mathfrak{L}}\left(r_{k}\right), \xi_{\Delta_{\mathfrak{L}}}\left(r_{k}\right)\right),\left(\Phi_{\mathfrak{L}}\left(r_{k}\right), \xi_{\Phi_{\mathfrak{L}}}\left(r_{k}\right)\right)\right\rangle$ & $\left\langle\left(\rho_{\mathfrak{L}}^{k}, \xi_{\rho_{\mathfrak{L}}^{k}}\right),\left(\sigma_{\mathfrak{L}}^{k}, \xi_{\sigma_{\mathfrak{L}}^{k}}\right)\right\rangle$ \\
\hline$P$ (Patient) & $s_{1}$ & $\langle(0.8,0.7),(0.3,0.4)\rangle$ & $\langle(0.6,0.6),(0.3,0.2)\rangle$ \\
& $s_{2}$ & $\langle(0.8,0.9),(0.3,0.3)\rangle$ & $\langle(0.7,0.8),(0.1,0.2)\rangle$ \\
& $s_{3}$ & $\langle(0.2,0),(0.9,0.8)\rangle$ & $\langle(0.3,0.1),(0.7,0.8)\rangle$ \\
& $s_{4}$ & $\langle(0.5,0.6),(0.4,0.3)\rangle$ & $\langle(0.5,0.4),(0.2,0.1)\rangle$ \\
& $s_{5}$ & $\langle(0.4,0.4),(0.3,0.2)\rangle$ & $\langle(0.4,0.5),(0.3,0.3)\rangle$ \\
\hline
\end{tabular}

Table 4 CLDFSs proposed a medical expert for diseases $D_{1}, D_{2}, D_{3}, D_{4}$ and $D_{5}$

\begin{tabular}{|c|c|c|c|}
\hline & & $\left\langle\left(\Delta_{\mathfrak{L}}\left(r_{k}\right), \xi_{\Delta_{\mathfrak{L}}}\left(r_{k}\right)\right),\left(\Phi_{\mathfrak{L}}\left(r_{k}\right), \xi_{\Phi_{\mathfrak{L}}}\left(r_{k}\right)\right)\right\rangle$ & $\left\langle\left(\rho_{\mathfrak{L}}^{k}, \xi_{\rho_{\mathfrak{L}}^{k}}\right),\left(\sigma_{\mathfrak{L}}^{k}, \xi_{\sigma_{\mathfrak{L}}^{k}}\right)\right\rangle$ \\
\hline \multirow[t]{5}{*}{$D_{1}$ (Malaria) } & $s_{1}$ & $\langle(0.4,0.5),(0.6,0.5)\rangle$ & $\langle(0.3,0.3),(0.5,0.6)\rangle$ \\
\hline & $s_{2}$ & $\langle(0.2,0.4),(0.7,0.6)\rangle$ & $\langle(0.2,0),(0.7,1)\rangle$ \\
\hline & $s_{3}$ & $\langle(0.3,0.4),(0.8,0.2)\rangle$ & $\langle(0.1,0.4),(0.6,0.5)\rangle$ \\
\hline & $s_{4}$ & $\langle(0.7,0.8),(0.3,0.3)\rangle$ & $\langle(0.8,0.6),(0.2,0.3)\rangle$ \\
\hline & $s_{5}$ & $\langle(0.9,0.5),(0.4,0.4)\rangle$ & $\langle(0.6,0.6),(0.2,0.4)\rangle$ \\
\hline \multirow[t]{5}{*}{$D_{2}$ (Typhoid) } & $s_{1}$ & $\langle(0.7,0.7),(0.5,0.4)\rangle$ & $\langle(0.6,0.6),(0.3,0.4)\rangle$ \\
\hline & $s_{2}$ & $\langle(0.1,0.1),(0.8,0.9)\rangle$ & $\langle(0.3,0.3),(0.2,0.1)\rangle$ \\
\hline & $s_{3}$ & $\langle(0.8,0.7),(0.5,0.4)\rangle$ & $\langle(0.7,0.4),(0.3,0.4)\rangle$ \\
\hline & $s_{4}$ & $\langle(0.9,0.7),(0.2,0.5)\rangle$ & $\langle(0.6,0.6),(0.2,0.3)\rangle$ \\
\hline & $s_{5}$ & $\langle(0.8,0.7),(0.1,0)\rangle$ & $\langle(0.7,0.9),(0.2,0.1)\rangle$ \\
\hline \multirow[t]{5}{*}{$D_{3}$ (Viral Fever) } & $s_{1}$ & $\langle(0.4,0.2),(0.7,0.8)\rangle$ & $\langle(0.4,0.2),(0.5,0.6)\rangle$ \\
\hline & $s_{2}$ & $\langle(0.2,0.4),(0.6,0.9)\rangle$ & $\langle(0.3,0.1),(0.6,0.7)\rangle$ \\
\hline & $s_{3}$ & $\langle(0.3,0.7),(0.8,0.9)\rangle$ & $\langle(0.3,0.5),(0.7,0.2)\rangle$ \\
\hline & $s_{4}$ & $\langle(1,0.6),(0.4,0.3)\rangle$ & $\langle(1,0.7),(0,0)\rangle$ \\
\hline & $s_{5}$ & $\langle(0.6,0.7),(0.5,0.8)\rangle$ & $\langle(0.7,0.8),(0.2,0.2)\rangle$ \\
\hline \multirow[t]{5}{*}{$D_{4}$ (Chest Problem) } & $s_{1}$ & $\langle(0.9,0.7),(0.4,0.4)\rangle$ & $\langle(0.7,0.6),(0.3,0.3)\rangle$ \\
\hline & $s_{2}$ & $\langle(0.9,0.9),(0.2,0.4)\rangle$ & $\langle(0.9,0.7),(0.1,0)\rangle$ \\
\hline & $s_{3}$ & $\langle(0.2,0.2),(0.8,0.7)\rangle$ & $\langle(0.3,0.2),(0.6,0.8)\rangle$ \\
\hline & $s_{4}$ & $\langle(0.5,0.5),(0.5,0.3)\rangle$ & $\langle(0.5,0.3),(0.1,0.1)\rangle$ \\
\hline & $s_{5}$ & $\langle(0.5,0.4),(0.2,0.3)\rangle$ & $\langle(0.4,0.5),(0.3,0.4)\rangle$ \\
\hline \multirow[t]{5}{*}{$D_{5}($ Stomach Problem $)$} & $s_{1}$ & $\langle(0.4,0.5),(0.5,0.4)\rangle$ & $\langle(0.5,0.7),(0.4,0.4)\rangle$ \\
\hline & $s_{2}$ & $\langle(0.3,0.3),(0.8,0.9)\rangle$ & $\langle(0.4,0.5),(0.6,0.3)\rangle$ \\
\hline & $s_{3}$ & $\langle(0.9,0.8),(0.4,0.4)\rangle$ & $\langle(0.9,1),(0.1,0)\rangle$ \\
\hline & $s_{4}$ & $\langle(0.5,0.3),(0.6,0.5)\rangle$ & $\langle(0.4,0.5),(0.4,0.6)\rangle$ \\
\hline & $s_{5}$ & $\langle(0.6,0.2),(0.5,0.2)\rangle$ & $\langle(0.5,0.6),(0.4,0.4)\rangle$ \\
\hline
\end{tabular}

Table 5 WCSMs for weight vectors $\varpi^{i}$

\begin{tabular}{|c|c|c|c|c|c|}
\hline & $\mathfrak{C}_{\text {sim }}^{\varpi^{j}}\left(P, D_{1}\right)$ & $\mathfrak{C}_{\text {sim }}^{\varpi^{j}}\left(P, D_{2}\right)$ & $\mathfrak{C}_{\operatorname{sim}}^{\varpi^{j}}\left(P, D_{3}\right)$ & $\mathfrak{C}_{\text {sim }}^{\varpi^{j}}\left(P, D_{4}\right)$ & $\mathfrak{C}_{s i m}^{\varpi^{j}}\left(P, D_{5}\right)$ \\
\hline$\varpi^{1}=(0.2,0.2,0.2,0.2,0.2)^{T}$ & 0.8213 & 0.7636 & 0.7995 & 0.9866 & 0.7716 \\
\hline$\varpi^{2}=(0.1,0.3,0.3,0.1,0.2)^{T}$ & 0.7741 & 0.7117 & 0.7704 & 0.9853 & 0.7029 \\
\hline$\varpi^{3}=(0.4,0.1,0.1,0.1,0.3)^{T}$ & 0.8529 & 0.7916 & 0.8083 & 0.9889 & 0.8567 \\
\hline$\varpi^{4}=(0.1,0.1,0.4,0.3,0.1)^{T}$ & 0.8602 & 0.7714 & 0.8321 & 0.9864 & 0.6832 \\
\hline$\varpi^{5}=(0.3,0.3,0.2,0.1,0.1)^{T}$ & 0.7608 & 0.7083 & 0.744 & 0.9873 & 0.7506 \\
\hline$\varpi^{6}=(0.01,0.01,0.01,0.01,0.96)^{T}$ & 0.9501 & 0.8941 & 0.924 & 0.9847 & 0.9534 \\
\hline
\end{tabular}


Fig. 2 The portrayal of WCSMs for weight vectors $\varpi^{i}$

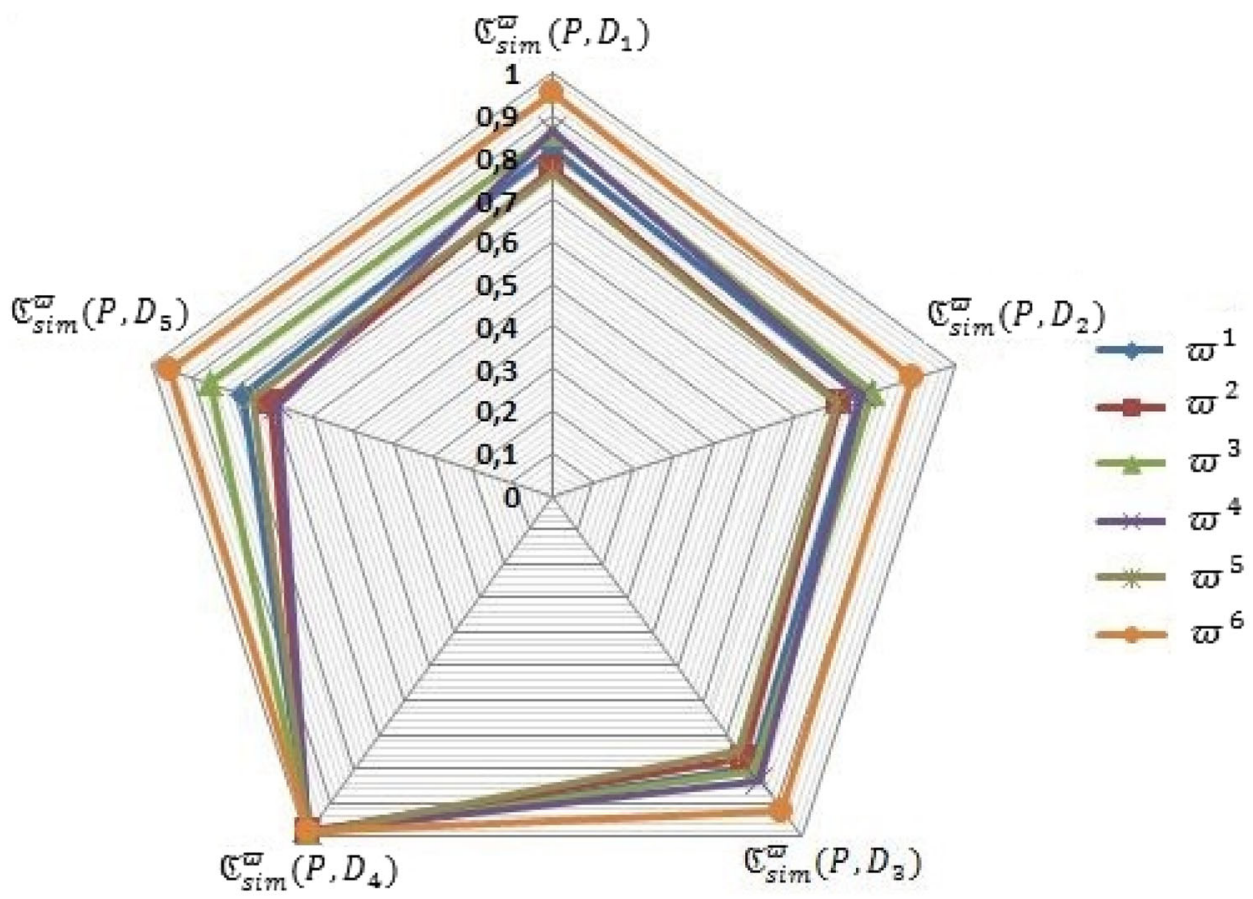

\section{Comparative study}

In this section, we test the performance of the cosine similarity measures and cosine distance measures based on the CLDFS in dealing with different practical application problems that are solved by using the similarity and distance measures on the fuzzy sets and extensions. Thus, we confirm the superiority of the the emerging (weighted) cosine similarity and distance measures by comparing with the existing (weighted) similarity and distance measures based on IFS, PyFS, q-ROFS, CIFS, and CPyFS.

(1) Any $\operatorname{IFN}\left(\left\langle\Delta_{\mathcal{I}}, \Phi_{\mathcal{I}}\right\rangle\right)$ (where $0 \leq \Delta_{\mathcal{I}}+\Phi_{\mathcal{I}} \leq$ 1) can be considered as $\operatorname{CLDFN}\left(\left\langle\left(\Delta_{\mathcal{I}}, 0\right),\left(\Phi_{\mathcal{I}}, 0\right)\right\rangle\right.$, $\langle(0,0),(0,0)\rangle)$, i.e., $\left(\left\langle\left(\Delta_{\mathcal{I}}, 0\right),\left(\Phi_{\mathcal{I}}, 0\right)\right\rangle,\langle(0,0),(0,0)\rangle\right)$ corresponds to $\left(\left\langle\Delta_{\mathcal{I}}, \Phi_{\mathcal{I}}\right\rangle\right)$. Using the matching of IFN and CLDFN, we endeavor to compare the cosine similarity measure and weighted cosine similarity measure in Eqs. (4.2) and (4.3) with the (weighted) similarity measures proposed for IFS. These comparisons are given in Table 11.

(2) Any PyFN $\left(\left\langle\Delta_{\mathcal{P}}, \Phi_{\mathcal{P}}\right\rangle\right)\left(\right.$ where $0 \leq\left(\Delta_{\mathcal{P}}\right)^{2}+\left(\Phi_{\mathcal{P}}\right)^{2} \leq$ $1)$ is considered as $\operatorname{CLDFN}\left(\left\langle\left(\Delta_{\mathcal{P}}, 0\right),\left(\Phi_{\mathcal{P}}, 0\right)\right\rangle\right.$, $\langle(0,0),(0,0)\rangle)$, i.e., $\left(\left\langle\left(\Delta_{\mathcal{P}}, 0\right),\left(\Phi_{\mathcal{P}}, 0\right)\right\rangle,\langle(0,0),(0,0)\rangle\right)$ corresponds to $\left(\left\langle\Delta_{\mathcal{P}}, \Phi_{\mathcal{P}}\right\rangle\right)$. Now, let us compare the cosine similarity measure and weighted cosine similarity measure in Eqs. (4.2) and (4.3) with the (weighted) similarity measures proposed for PyFS. Table 12 is created to showcase these comparisons.
(3) Any q-ROFN $\left(\left\langle\Delta_{\mathcal{Q}}, \Phi_{\mathcal{Q}}\right\rangle\right)$ (where $0 \leq\left(\Delta_{\mathcal{Q}}\right)^{q}+$ $\left.\left(\Phi_{\mathcal{Q}}\right)^{q} \leq 1\right)$ can be considered as CLDFN $\left(\left\langle\left(\Delta_{\mathcal{Q}}, 0\right),\left(\Phi_{\mathcal{Q}}, 0\right)\right\rangle,\langle(0,0),(0,0)\rangle\right)$, i.e., $\quad\left(\left\langle\left(\Delta_{\mathcal{Q}}, 0\right)\right.\right.$, $\left.\left.\left(\Phi_{\mathcal{Q}}, 0\right)\right\rangle,\langle(0,0),(0,0)\rangle\right)$ corresponds to $\left(\left\langle\Delta_{\mathcal{Q}}, \Phi_{\mathcal{Q}}\right\rangle\right)$. As a result of comparing the cosine similarity measure and weighted cosine similarity measure in Eqs. (4.2) and (4.3) with the (weighted) similarity measures proposed for q-ROFS, Table 13 is created.

(4) Any $\operatorname{CIFN}\left(\left\langle\left(\Delta_{\mathfrak{I}}, \xi_{\Delta_{\mathfrak{I}}}\right)\left(\Phi_{\mathfrak{I}}, \xi_{\Phi_{\mathfrak{I}}}\right)\right\rangle\right.$ ) (where $0 \leq \Delta_{\mathfrak{I}}+$ $\Phi_{\mathfrak{I}} \leq 1$ and $\left.0 \leq \xi_{\Delta_{\mathfrak{I}}}+\xi_{\Phi_{\mathfrak{I}}} \leq 1\right)$ can be considered as $\operatorname{CLDFN}\left(\left\langle\left(\Delta_{\mathfrak{I}}, \xi_{\Delta_{\mathfrak{I}}}\right),\left(\Phi_{\mathfrak{I}}, \xi_{\Phi_{\mathfrak{I}}}\right)\right\rangle,\langle(0,0),(0,0)\rangle\right)$, i.e., $\left(\left\langle\left(\Delta_{\mathfrak{I}}, \xi_{\Delta_{\mathfrak{I}}}\right),\left(\Phi_{\mathfrak{I}}, \xi_{\Phi_{\mathfrak{I}}}\right)\right\rangle,\langle(0,0),(0,0)\rangle\right)$ corresponds to $\left(\left\langle\left(\Delta_{\mathfrak{I}}, \xi_{\Delta_{\mathfrak{I}}}\right)\left(\Phi_{\mathfrak{I}}, \xi_{\Phi_{\mathfrak{I}}}\right)\right\rangle\right)$. We give Table 14 to match some (weighted) similarity measures proposed for CIFS with the cosine similarity measure and weighted cosine similarity measure in Eqs. (4.2) and (4.3).

(5) Any $\operatorname{CPyFN}\left(\left\langle\left(\Delta_{\mathfrak{P}}, \xi_{\Delta_{\mathfrak{P}}}\right)\left(\Phi_{\mathfrak{P}}, \xi_{\Phi_{\mathfrak{P}}}\right)\right\rangle\right.$ ) (where $0 \leq$ $\left(\Delta_{\mathfrak{P}}\right)^{2}+\left(\Phi_{\mathfrak{P}}\right)^{2} \leq 1$ and $\left.0 \leq\left(\xi_{\Delta_{\mathfrak{P}}}\right)^{2}+\left(\xi_{\Phi_{\mathfrak{P}}}\right)^{2} \leq 1\right)$ can be considered as $\operatorname{CLDFN}\left(\left\langle\left(\Delta_{\mathfrak{I}}, \xi_{\Delta_{\mathfrak{P}}}\right),\left(\Phi_{\mathfrak{P}}, \xi_{\Phi_{\mathfrak{P}}}\right)\right\rangle\right.$, $\langle(0,0),(0,0)\rangle)$, i.e. $\left(\left\langle\left(\Delta_{\mathfrak{P}}, \xi_{\Delta_{\mathfrak{P}}}\right),\left(\Phi_{\mathfrak{P}}, \xi_{\Phi_{\mathfrak{P}}}\right)\right\rangle,\langle(0,0)\right.$, $(0,0)\rangle)$ corresponds to $\left(\left\langle\left(\Delta_{\mathfrak{P}}, \xi_{\Delta_{\mathfrak{P}}}\right)\left(\Phi_{\mathfrak{P}}, \xi_{\Phi_{\mathfrak{P}}}\right)\right\rangle\right)$. We compare the cosine distance measure and weighted cosine distance measure in Eqs. (4.4) and (4.5) with the (weighted) distance measures proposed for CPyFS. Table 15 is dedicated to these comparisons.

\section{Advantages of the proposed approaches:}

From the existing studies and the proposed approaches, we emphasize the following merits of the proposed ones to deal with the decision making under the CLDF environment. 
Table 6 CLDFS for patient $P^{*}$

Table 7 CLDFSs proposed by first expert for diseases $D_{1}^{*}, D_{2}^{*}, D_{3}^{*}$

Table 8 CLDFSs proposed by second expert for diseases $D_{1}^{*}, D_{2}^{*}, D_{3}^{*}$

Table 9 CLDFSs proposed by third expert for diseases $D_{1}^{*}, D_{2}^{*}, D_{3}^{*}$

\begin{tabular}{llll}
\hline & & $\left\langle\left(\Delta_{\mathfrak{L}}\left(s_{k}^{*}\right), \xi_{\Delta_{\mathfrak{L}}}\left(s_{k}^{*}\right)\right),\left(\Phi_{\mathfrak{L}}\left(s_{k}^{*}\right), \xi_{\Phi_{\mathfrak{L}}}\left(s_{k}^{*}\right)\right)\right\rangle$ & $\left\langle\left(\rho_{\mathfrak{L}}^{k}, \xi_{\rho_{\mathfrak{L}}^{k}}\right),\left(\sigma_{\mathfrak{L}}^{k}, \xi_{\sigma_{\mathfrak{L}}^{k}}\right)\right\rangle$ \\
\hline$P^{*}$ (Patient) & $s_{1}^{*}$ & $\langle(0.4,0.3),(0.6,0.4)\rangle$ & $\langle(0.5,0.3),(0.5,0.4)\rangle$ \\
& $s_{2}^{*}$ & $\langle(0.7,0.9),(0.3,0.2)\rangle$ & $\langle(0.6,0.8),(0.3,0.2)\rangle$ \\
& $s_{3}^{*}$ & $\langle(0.8,0.4),(0.3,0.4)\rangle$ & $\langle(0.9,0.7),(0.1,0.2)\rangle$ \\
\hline
\end{tabular}

\begin{tabular}{llll}
\hline & & $\left\langle\left(\Delta_{\mathfrak{L}}\left(s_{k}^{*}\right), \xi_{\Delta_{\mathfrak{L}}}\left(s_{k}^{*}\right)\right),\left(\Phi_{\mathfrak{L}}\left(s_{k}^{*}\right), \xi_{\Phi_{\mathfrak{L}}}\left(s_{k}^{*}\right)\right)\right\rangle$ & $\left\langle\left(\rho_{\mathfrak{L}}^{k}, \xi_{\rho_{\mathfrak{L}}^{k}}\right),\left(\sigma_{\mathfrak{L}}^{k}, \xi_{\sigma_{\mathfrak{L}}^{k}}\right)\right\rangle$ \\
\hline$D_{1}^{*}$ (Malaria) & $s_{1}^{*}$ & $\langle(0.7,0.8),(0.3,0.4)\rangle$ & $\langle(0.6,0.8),(0.2,0.1)\rangle$ \\
& $s_{2}^{\dagger}$ & $\langle(0.8,0.4),(0.2,0.5)\rangle$ & $\langle(0.7,0.6),(0.2,0.3)\rangle$ \\
& $s_{3}^{*}$ & $\langle(0.7,0.7),(0.1,0.1)\rangle$ & $\langle(0.8,0.5),(0.2,0.3)\rangle$ \\
$D_{2}^{*}$ (Typhoid) & $s_{1}^{*}$ & $\langle(0.3,0.3),(0.7,0.9)\rangle$ & $\langle(0.4,0.5),(0.6,0.4)\rangle$ \\
& $s_{2}^{*}$ & $\langle(0.8,0.7),(0.4,0.4)\rangle$ & $\langle(0.6,0.7),(0.3,0.3)\rangle$ \\
$D_{3}^{*}$ (Viral Fever) & $s_{3}^{*}$ & $\langle(0.9,0.6),(0.3,0.5)\rangle$ & $\langle(0.4,0.3),(0.6,0.6)\rangle$ \\
& $s_{1}^{*}$ & $\langle(0.4,0.1),(0.7,0.8)\rangle$ & $\langle(0.8,0.4),(0.1,0)\rangle$ \\
& $s_{2}^{*}$ & $\langle(0.9,0.9),(0.3,0.4)\rangle$ & $\langle(0.4,0.5),(0.5,0.4)\rangle$ \\
\hline
\end{tabular}

\begin{tabular}{llll}
\hline & & $\left\langle\left(\Delta_{\mathfrak{L}}\left(s_{k}^{*}\right), \xi_{\Delta_{\mathfrak{L}}}\left(s_{k}^{*}\right)\right),\left(\Phi_{\mathfrak{L}}\left(s_{k}^{*}\right), \xi_{\Phi_{\mathfrak{L}}}\left(s_{k}^{*}\right)\right)\right\rangle$ & $\left\langle\left(\rho_{\mathfrak{L}}^{k}, \xi_{\rho_{\mathfrak{L}}^{k}}\right),\left(\sigma_{\mathfrak{L}}^{k}, \xi_{\sigma_{\mathfrak{L}}^{k}}\right)\right\rangle$ \\
\hline$D_{1}^{*}$ (Malaria) & $s_{1}^{*}$ & $\langle(0.8,0.8),(0.3,0.4)\rangle$ & $\langle(0.7,0.8),(0.2,0.1)\rangle$ \\
& $s_{2}^{*}$ & $\langle(0.8,0.6),(0.1,0.2)\rangle$ & $\langle(0.7,0.6),(0.2,0.2)\rangle$ \\
& $s_{3}^{*}$ & $\langle(0.7,0.8),(0.5,0.1)\rangle$ & $\langle(0.7,1),(0.2,0)\rangle$ \\
$D_{2}^{*}$ (Typhoid) & $s_{1}^{*}$ & $\langle(0.2,0.4),(0.6,0.6)\rangle$ & $\langle(0.3,0.4),(0.6,0.5)\rangle$ \\
& $s_{2}^{*}$ & $\langle(0.6,0.7),(0.5,0.6)\rangle$ & $\langle(0.5,0.4),(0.3,0.5)\rangle$ \\
& $s_{3}^{*}$ & $\langle(0.7,0.8),(0.3,0.4)\rangle$ & $\langle(0.8,0.6),(0.1,0.3)\rangle$ \\
$D_{3}^{*}$ (Viral Fever) & $s_{1}^{*}$ & $\langle(0.4,0.2),(0.7,0.8)\rangle$ & $\langle(0.4,0.2),(0.5,0.6)\rangle$ \\
& $s_{2}^{*}$ & $\langle(0.8,1),(0.2,0.4)\rangle$ & $\langle(0.8,0.5),(0.2,0.3)\rangle$ \\
& $s_{3}^{*}$ & $\langle(0.6,0.8),(0.4,0.6)\rangle$ & $\langle(0.5,0.2),(0.4,0.8)\rangle$ \\
\hline
\end{tabular}

\begin{tabular}{llll}
\hline & & $\left\langle\left(\Delta_{\mathfrak{L}}\left(s_{k}^{*}\right), \xi_{\Delta_{\mathfrak{L}}}\left(s_{k}^{*}\right)\right),\left(\Phi_{\mathfrak{L}}\left(s_{k}^{*}\right), \xi_{\Phi_{\mathfrak{L}}}\left(s_{k}^{*}\right)\right)\right\rangle$ & $\left\langle\left(\rho_{\mathcal{L}}^{k}, \xi_{\rho_{\mathfrak{L}}^{k}}\right),\left(\sigma_{\mathfrak{L}}^{k}, \xi_{\sigma_{\mathfrak{L}}^{k}}\right)\right\rangle$ \\
\hline$D_{1}^{*}$ (Malaria) & $s_{1}^{*}$ & $\langle(0.9,0.5),(0.3,0.5)\rangle$ & $\langle(0.8,0.5),(0.2,0.5)\rangle$ \\
& $s_{2}^{*}$ & $\langle(0.7,0.4),(0.4,0.4)\rangle$ & $\langle(0.6,0.4),(0.3,0.5)\rangle$ \\
& $s_{3}^{*}$ & $\langle(0.8,0.2),(0.5,0.5)\rangle$ & $\langle(0.6,0.3),(0.3,0.3)\rangle$ \\
$D_{2}^{*}$ (Typhoid) & $s_{1}^{*}$ & $\langle(0.5,0.5),(0.3,0.7)\rangle$ & $\langle(0.5,1),(0.2,0)\rangle$ \\
& $s_{2}^{*}$ & $\langle(0.6,0.6),(0.4,0.5)\rangle$ & $\langle(0.6,0.5),(0.2,0.1)\rangle$ \\
& $s_{3}^{*}$ & $\langle(0.7,0.9),(0.3,0.5)\rangle$ & $\langle(0.8,0.3),(0.2,0.1)\rangle$ \\
& $s_{1}^{*}$ (Viral Fever) & $\langle(0.5,0),(0.8,0.5)\rangle$ & $\langle(1,0.7),(0.5,0)\rangle$ \\
& $s_{2}^{*}$ & $\langle(0.8,0.3),(0.2,0.4)\rangle$ & $\langle(0.6,0.4),(0.4,0.6)\rangle$ \\
\hline
\end{tabular}


Table 10 CLDFS obtained by aggregating experts' evaluations (with $\oplus$ )

\begin{tabular}{llll}
\hline & & $\left\langle\left(\Delta_{\mathfrak{L}}\left(s_{k}^{*}\right), \xi_{\Delta_{\mathfrak{L}}}\left(s_{k}^{*}\right)\right),\left(\Phi_{\mathfrak{L}}\left(s_{k}^{*}\right), \xi_{\Phi_{\mathfrak{L}}}\left(s_{k}^{*}\right)\right)\right\rangle$ & $\left\langle\left(\rho_{\mathfrak{L}}^{k}, \xi_{\rho_{\mathfrak{L}}^{k}}\right),\left(\sigma_{\mathfrak{L}}^{k}, \xi_{\sigma_{\mathfrak{L}}^{k}}\right)\right\rangle$ \\
\hline$D_{1}^{*}$ (Malaria) & $s_{1}^{*}$ & $\langle(0.994,0.98),(0.027,0.08)\rangle$ & $\langle(0.976,0.98),(0.008,0.005)\rangle$ \\
& $s_{2}^{*}$ & $\langle(0.988,0.856),(0.008,0.04)\rangle$ & $\langle(0.964,0.904),(0.012,0.03)\rangle$ \\
$D_{2}^{*}$ (Typhoid) & $s_{3}^{*}$ & $\langle(0.982,0.952),(0.025,0.005)\rangle$ & $\langle(0.976,1),(0.012,0)\rangle$ \\
& $s_{2}^{*}$ & $\langle(0.72,0.79),(0.126,0.378)\rangle$ & $\langle(0.92,0.91),(0.018,0.015)\rangle$ \\
& $s_{3}^{*}$ & $\langle(0.968,0.964),(0.08,0.12)\rangle$ & $\langle(0.992,0.994),(0.002,0.006)\rangle$ \\
$D_{3}^{*}$ (Viral Fever) & $s_{1}^{*}$ & $\langle(0.991,0.992),(0.027,0.1)\rangle$ & $\langle(0.748,0.832),(0.15,0.072)\rangle$ \\
& $s_{2}^{*}$ & $\langle(0.82,0.28),(0.392,0.32)\rangle$ & $\langle(1,0.91),(0,0)\rangle$ \\
& $s_{3}^{*}$ & $\langle(0.996,1),(0.012,0.064)\rangle$ & $\langle(0.88,0.76),(0.08,0.192)\rangle$ \\
\hline
\end{tabular}

Table 11 Comparison of CLDFS-based CSM (or WCSM) with some of existing IFS-based SMs

\begin{tabular}{|c|c|c|c|}
\hline Problem & $\begin{array}{l}\text { Result of the pro- } \\
\text { posed IFS-based } \\
\text { SM }\end{array}$ & $\begin{array}{l}\text { Result of Our } \\
\text { CSM (or WCSM) }\end{array}$ & $\begin{array}{l}\text { Ranking for both SMs (IFS-based } \\
\text { SM and Our CSM) }\end{array}$ \\
\hline Example $8[10]$ & $\begin{array}{l}S_{Y}\left(A_{1}, B\right)=0.887 \\
S_{Y}\left(A_{2}, B\right)=0.913 \\
S_{Y}\left(A_{3}, B\right)=0.936\end{array}$ & $\begin{array}{l}\mathfrak{C}_{\text {sim }}\left(A_{1}, B\right)=0.9616 \\
\mathfrak{C}_{\text {sim }}\left(A_{2}, B\right)=0.9712 \\
\mathfrak{C}_{\text {sim }}\left(A_{3}, B\right)=0.98\end{array}$ & $\begin{array}{l}S_{Y}\left(A_{3}, B\right)>S_{Y}\left(A_{2}, B\right)> \\
S_{Y}\left(A_{1}, B\right) \mathfrak{C}_{\text {sim }}\left(A_{3}, B\right)> \\
\mathfrak{C}_{\text {sim }}\left(A_{2}, B\right)>\mathfrak{C}_{\text {sim }}\left(A_{1}, B\right)\end{array}$ \\
\hline Example $8[10] \varpi=(0.5,0.3,0.2)$ & $\begin{array}{l}S_{W Y}\left(A_{1}, B\right)=0.853 \\
S_{W Y}\left(A_{2}, B\right)=0.919 \\
S_{W Y}\left(A_{3}, B\right)=0.949\end{array}$ & $\begin{array}{l}\mathfrak{C}_{\text {sim }}^{\varpi}\left(A_{1}, B\right)=0.9527 \\
\mathfrak{C}_{\text {sim }}^{\varpi}\left(A_{2}, B\right)=0.9693 \\
\mathfrak{C}_{\text {sim }}^{\varpi}\left(A_{3}, B\right)=0.9827\end{array}$ & $\begin{array}{l}S_{W Y}\left(A_{3}, B\right)>S_{W Y}\left(A_{2}, B\right)> \\
S_{W Y}\left(A_{1}, B\right) \mathfrak{C}_{s i m}^{\sigma}\left(A_{3}, B\right)> \\
\mathfrak{C}_{\text {sim }}^{\sigma}\left(A_{2}, B\right)>\mathfrak{C}_{\text {sim }}^{\sigma}\left(A_{1}, B\right)\end{array}$ \\
\hline Example 1 [13] & $\begin{array}{l}C_{I F S}\left(C_{1}, Q\right)=0.9353 \\
C_{I F S}\left(C_{2}, Q\right)=0.9519 \\
C_{I F S}\left(C_{3}, Q\right)=0.9724\end{array}$ & $\begin{array}{l}\mathfrak{C}_{\text {sim }}\left(C_{1}, Q\right)=0.9353 \\
\mathfrak{C}_{\text {sim }}\left(C_{2}, Q\right)=0.9519 \\
\mathfrak{C}_{\text {sim }}\left(C_{3}, Q\right)=0.9724\end{array}$ & $\begin{array}{l}C_{I F S}\left(C_{3}, Q\right)>C_{I F S}\left(C_{2}, Q\right)> \\
C_{I F S}\left(C_{1}, Q\right) \mathfrak{C}_{s i m}\left(C_{3}, Q\right)> \\
\mathfrak{C}_{\text {sim }}\left(C_{2}, Q\right)>\mathfrak{C}_{\text {sim }}\left(C_{1}, Q\right) \\
\end{array}$ \\
\hline Example $1[13] \varpi=(0.5,0.3,0.2)$ & $\begin{array}{l}W_{I F S}\left(C_{1}, Q\right)=0.9133 \\
W_{I F S}\left(C_{2}, Q\right)=0.9404 \\
W_{I F S}\left(C_{3}, Q\right)=0.9712\end{array}$ & $\begin{array}{l}\mathfrak{C}_{\text {sim }}^{\sigma}\left(C_{1}, Q\right)=0.9133 \\
\mathfrak{C}_{\text {sim }}^{\sigma}\left(C_{2}, Q\right)=0.9404 \\
\mathfrak{C}_{\text {sim }}^{\sigma}\left(C_{3}, Q\right)=0.9712\end{array}$ & $\begin{array}{l}W_{I F S}\left(C_{3}, Q\right)>W_{I F S}\left(C_{2}, Q\right)> \\
W_{I F S}\left(C_{1}, Q\right) \mathfrak{C}_{\text {sim }}^{\sigma}\left(C_{3}, Q\right)> \\
\mathfrak{C}_{\text {sim }}^{\sigma}\left(C_{2}, Q\right)>\mathfrak{C}_{\text {sim }}^{\sigma}\left(C_{1}, Q\right)\end{array}$ \\
\hline Example $2[13]$ & $\begin{array}{l}C_{I F S}\left(P, Q_{1}\right)=0.9046 \\
C_{I F S}\left(P, Q_{2}\right)=0.8602 \\
C_{I F S}\left(P, Q_{3}\right)=0.851 \\
C_{I F S}\left(P, Q_{4}\right)=0.5033 \\
C_{I F S}\left(P, Q_{5}\right)=0.4542\end{array}$ & $\begin{array}{l}\mathfrak{C}_{\text {sim }}\left(P, Q_{1}\right)=0.9046 \\
\mathfrak{C}_{\text {sim }}\left(P, Q_{2}\right)=0.8602 \\
\mathfrak{C}_{\text {sim }}\left(P, Q_{3}\right)=0.851 \\
\mathfrak{C}_{\text {sim }}\left(P, Q_{4}\right)=0.5033 \\
\mathfrak{C}_{\text {sim }}\left(P, Q_{5}\right)=0.4542\end{array}$ & $\begin{array}{l}C_{I F S}\left(P, Q_{1}\right)>C_{I F S}\left(P, Q_{2}\right)> \\
C_{I F S}\left(P, Q_{3}\right)>C_{I F S}\left(P, Q_{4}\right)> \\
C_{I F S}\left(P, Q_{5}\right) \\
\mathfrak{C}_{\text {sim }}\left(P, Q_{1}\right)>\mathfrak{C}_{\text {sim }}\left(P, Q_{2}\right)> \\
\mathfrak{C}_{\text {sim }}\left(P, Q_{3}\right)>\mathfrak{C}_{\text {sim }}\left(P, Q_{4}\right)> \\
\mathfrak{C}_{\text {sim }}\left(P, Q_{5}\right)\end{array}$ \\
\hline
\end{tabular}

- A complex linear Diophantine fuzzy set is a generalization of the existing studies such as fuzzy set [1], complex fuzzy set [51], intuitionistic fuzzy set [6], complex intuitionistic fuzzy set [54,55], Pythagorean fuzzy set [14], complex Pythagorean fuzzy set [60], q-rung orthopair fuzzy set [26], complex q-rung orthopair fuzzy set [61], and linear Diophantine fuzzy set [65] by considering much more information regarding an object during the process and to deal with the two-dimensional information in a set. This generalized form achieves new solutions for problems based on fuzzy sets and their extensions in many fields (as well as decision-making).

- The CLDFS (with the grades of membership, nonmembership and reference parameters are complexvalued) contains information that covers more amplitude and phase terms than the FS (with the grade of membership is real-valued), CFS (with the grade of membership is complex-valued), IFS (or PyFS, q-ROFS) (with the grades of membership and non-membership are real-valued), CIFS (or CPyFS, Cq-ROFS) (with 


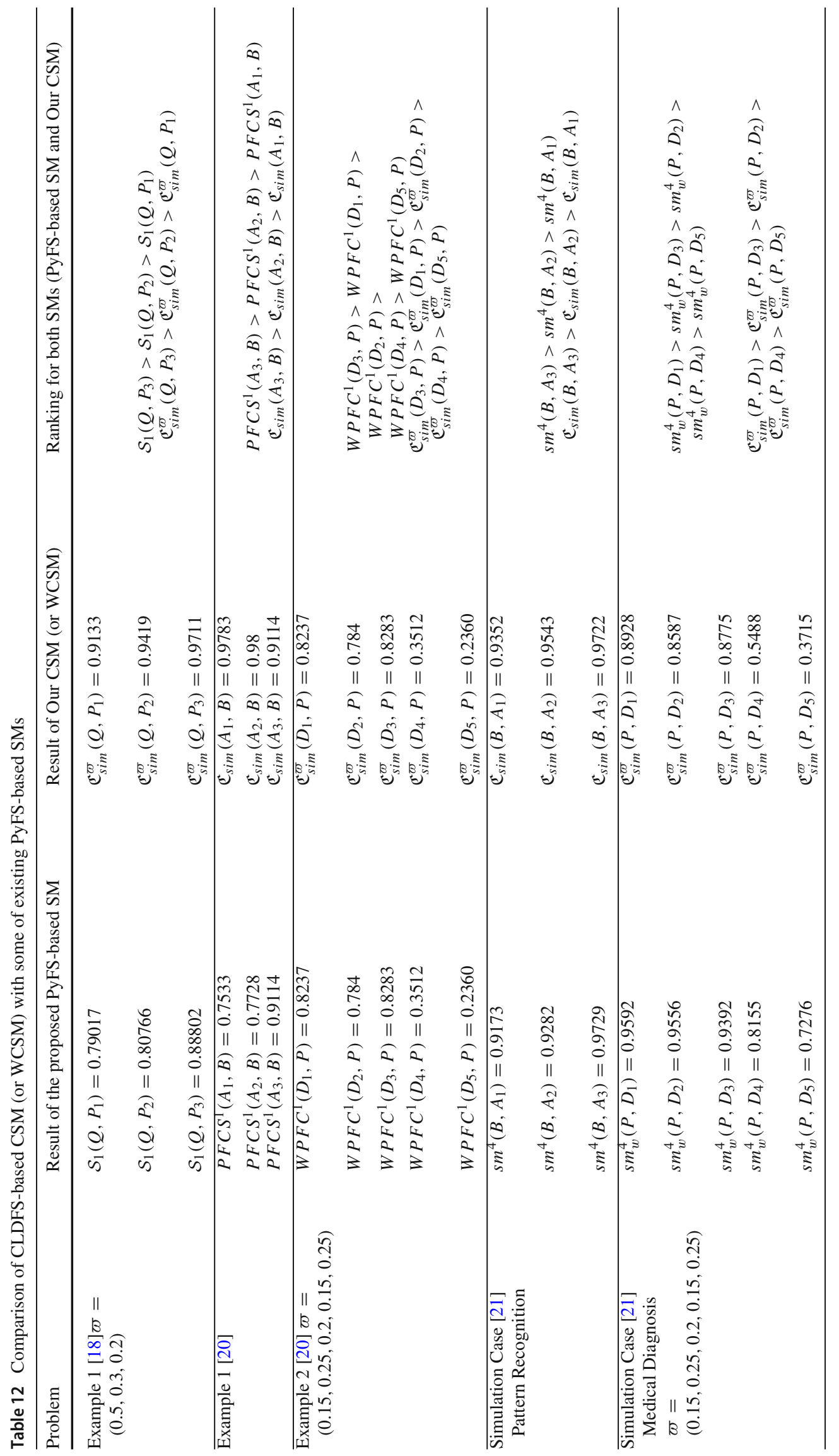


Table 13 Comparison of CLDFS-based CSM (or WCSM) with some of existing q-ROFS-based SMs

\begin{tabular}{|c|c|c|c|}
\hline Problem & $\begin{array}{l}\text { Result of the proposed } \\
\text { q-ROFS-based SM }\end{array}$ & $\begin{array}{l}\text { Result of Our CSM } \\
\text { (or WCSM) }\end{array}$ & $\begin{array}{l}\text { Ranking for both SMs (q-ROFS-based SM and Our } \\
\text { CSM) }\end{array}$ \\
\hline \multirow[t]{5}{*}{ Example 1 [29] } & $q-\operatorname{ROFC} C^{1}\left(A_{1}, A\right)=0.744$ & $\mathfrak{C}_{\text {sim }}\left(A_{1}, A\right)=0.899$ & \multirow{5}{*}{$\begin{array}{l}q-\operatorname{ROFC} C^{1}\left(A_{2}, A\right)>q-\operatorname{ROFC} C^{1}\left(A_{3}, A\right)>q- \\
\quad \operatorname{ROFC} C^{1}\left(A_{1}, A\right)> \\
q-\operatorname{ROFC} C^{1}\left(A_{4}, A\right)>q-R O F C^{1}\left(A_{5}, A\right) \\
\mathfrak{C}_{\text {sim }}\left(A_{2}, A\right)>\mathfrak{C}_{\text {sim }}\left(A_{3}, A\right)>\mathfrak{C}_{\text {sim }}\left(A_{1}, A\right)> \\
\quad \mathfrak{C}_{\text {sim }}\left(A_{4}, A\right)>\mathfrak{C}_{\text {sim }}\left(A_{5}, A\right)\end{array}$} \\
\hline & $q-\operatorname{ROFC} C^{1}\left(A_{2}, A\right)=0.803$ & $\mathfrak{C}_{\text {sim }}\left(A_{2}, A\right)=0.949$ & \\
\hline & $q-\operatorname{ROFC} C^{1}\left(A_{3}, A\right)=0.798$ & $\mathfrak{C}_{\text {sim }}\left(A_{3}, A\right)=0.938$ & \\
\hline & $q-\operatorname{ROFC} C^{1}\left(A_{4}, A\right)=0.734$ & $\mathfrak{C}_{\text {sim }}\left(A_{4}, A\right)=0.895$ & \\
\hline & $q-\operatorname{ROFC} C^{1}\left(A_{5}, A\right)=0.689$ & $\mathfrak{C}_{\text {sim }}\left(A_{5}, A\right)=0.885$ & \\
\hline \multirow{5}{*}{$\begin{array}{l}\text { Example } 2[29] \varpi= \\
\quad(0.15,0.2,0.25,0.1,0.3)\end{array}$} & $q-R O F W C^{1}\left(A_{1}, A\right)=0.57$ & $\mathfrak{C}_{\text {sim }}^{\sigma}\left(A_{1}, A\right)=0.831$ & \\
\hline & $q-R O F W C^{1}\left(A_{2}, A\right)=0.796$ & $\mathfrak{C}_{\text {sim }}^{\varpi}\left(A_{2}, A\right)=0.918$ & \multirow{4}{*}{$\begin{array}{l}q-R O F W C^{1}\left(A_{3}, A\right)>q-R O F W C^{1}\left(A_{5}, A\right)> \\
q-R O F W C^{1}\left(A_{2}, A\right)> \\
q-R O F W C^{1}\left(A_{4}, A\right)>q-R O F W C^{1}\left(A_{1}, A\right) \\
\mathfrak{C}_{\text {sim }}^{\sigma}\left(A_{3}, A\right)>\mathfrak{C}_{\text {sim }}^{\sigma}\left(A_{5}, A\right)>\mathfrak{C}_{\text {sim }}^{\sigma}\left(A_{2}, A\right)> \\
\mathfrak{C}_{\text {sim }}^{\sigma}\left(A_{4}, A\right)>\mathfrak{C}_{\text {sim }}^{\sigma}\left(A_{1}, A\right)\end{array}$} \\
\hline & $q-\operatorname{ROFWC} C^{1}\left(A_{3}, A\right)=0.995$ & $\mathfrak{C}_{s i m}^{\sigma \sigma}\left(A_{3}, A\right)=0.983$ & \\
\hline & $q-R O F W C^{1}\left(A_{4}, A\right)=0.716$ & $\mathfrak{C}_{s i m}^{\sigma}\left(A_{4}, A\right)=0.893$ & \\
\hline & $q-R O F W C^{1}\left(A_{5}, A\right)=0.8$ & $\mathfrak{C}_{\text {sim }}^{\Phi}\left(A_{5}, A\right)=0.929$ & \\
\hline
\end{tabular}

the grades of membership and non-membership are complex-valued), and LDFS (with the grades of membership, non-membership and reference parameters are real-valued). Therefore, the proposed similarity measures and distance measures under CLDFSs environment are improved versions of the current similarity measures and distance measures in [10,13,18,20,21,29,57,60].

- The major advantages of the proposed approaches are to consider the much more information with reference parameters to access the alternative to reduce the information loss. In addition, the similarity and distance measures based on the optimistic and pessimistic with or without weighting factor will help the decision-maker(s) to determine the optimal alternative(s) more precisely. In other words, we assert that the emerging similarity and distance measures will give the various options to the decision-makers based on their optimistic and pessimistic behavior in the decision-making process.

\section{Conclusion}

The decisive advantage of CLDFS is that one can freely choose the grades of complex-valued membership and complex-valued non-membership without any conditions. Therefore, it is put forward that the space of CLDFS is larger than the spaces of FS, IFS, PyFS, q-ROFS, CFS, CIFS, CPyFS, and Cq-ROFS. In addition to this, the CLDFS is more efficient and flexible rather than other set approaches due to the role of complex-valued reference parameters. These point to the efficiency and practicality of CLDFS for real-world issues. As the CLDFS provides a flexible and convenient way for people to express information and make decisions, it can be successfully applied to solve various decision-making problems. The CLDFS and its application to practical complex qualitative decision problems are an important research topic with broad application prospects and deep social significance. Broadly stated, some further research directions can be considered into the following subjects:

1. Information fusion for CLDFS.

- Investigating the operation rules and the comparable rules of CLDF information.

- Developing the aggregation operators of CLDF information. For example, each of CLDF power aggregation operators, CLDF neutral aggregation operators, CLDF Einstein aggregation operators, CLDF Dombi aggregation operators, CLDF Maclaurin aggregation operators, and CLDF Heronian aggregation operators will be a topic of interest in the near future.

2. Information measures for CLDFS.

- Proposing the novel systematic transformation of information measures (correlation coefficients, similarity measures, distance measures, inclusion measure, and entropy) for CLDFSs. 
Table 14 Comparison of CLDFS-based CSM (or WCSM) with some of existing CIFS-based SMs

\begin{tabular}{|c|c|c|c|}
\hline Problem & $\begin{array}{l}\text { Result of the } \\
\text { proposed CIFS- } \\
\text { based SM }\end{array}$ & Result of Our CSM (or WCSM) & $\begin{array}{l}\text { Ranking for both SMs (CIFS-based SM and Our } \\
\text { CSM) }\end{array}$ \\
\hline \multirow[t]{5}{*}{ Example 1 [57] } & $K_{1}\left(A_{1}, B\right)=0.8939$ & $\mathfrak{C}_{\text {sim }}\left(A_{1}, B\right)=0.9273$ & \\
\hline & $K_{1}\left(A_{2}, B\right)=0.9068$ & $\mathfrak{C}_{\text {sim }}\left(A_{2}, B\right)=0.9107$ & $\begin{array}{l}K_{1}\left(A_{3}, B\right)>K_{1}\left(A_{2}, B\right)>K_{1}\left(A_{1}, B\right)> \\
\quad K_{1}\left(A_{4}, B\right)>K_{1}\left(A_{5}, B\right)\end{array}$ \\
\hline & $K_{1}\left(A_{3}, B\right)=0.9424$ & $\mathfrak{C}_{\text {sim }}\left(A_{3}, B\right)=0.9576$ & \\
\hline & $K_{1}\left(A_{4}, B\right)=0.8830$ & $\mathfrak{C}_{s i m}\left(A_{4}, B\right)=0.9011$ & $\begin{array}{l}\mathfrak{C}_{\text {sim }}\left(A_{3}, B\right)>\mathfrak{C}_{\text {sim }}\left(A_{1}, B\right)>\mathfrak{C}_{\text {sim }}\left(A_{2}, B\right)> \\
\quad \mathfrak{C}_{\text {sim }}\left(A_{4}, B\right)>\mathfrak{C}_{\text {sim }}\left(A_{5}, B\right)\end{array}$ \\
\hline & $K_{1}\left(A_{5}, B\right)=0.8450$ & $\mathfrak{C}_{\text {sim }}\left(A_{5}, B\right)=0.8523$ & \\
\hline \multirow{5}{*}{$\begin{array}{l}\text { Example } 1[57] \varpi= \\
\quad(0.3,0.25,0.15,0.3)\end{array}$} & $K_{3}\left(A_{1}, B\right)=0.8965$ & $\mathfrak{C}_{\text {sim }}^{\sigma}\left(A_{1}, B\right)=0.9328$ & \\
\hline & $K_{3}\left(A_{2}, B\right)=0.9087$ & $\mathfrak{C}_{\text {sim }}^{\sigma}\left(A_{2}, B\right)=0.9094$ & $\begin{array}{l}K_{3}\left(A_{3}, B\right)>K_{3}\left(A_{2}, B\right)>K_{3}\left(A_{1}, B\right)> \\
\quad K_{3}\left(A_{4}, B\right)>K_{3}\left(A_{5}, B\right)\end{array}$ \\
\hline & $K_{3}\left(A_{3}, B\right)=0.9439$ & $\mathfrak{C}_{\text {sim }}^{\sigma}\left(A_{3}, B\right)=0.9571$ & \\
\hline & $K_{3}\left(A_{4}, B\right)=0.8747$ & $\mathfrak{C}_{\text {sim }}^{\sigma \sigma}\left(A_{4}, B\right)=0.8910$ & $\begin{array}{l}\mathfrak{C}_{\text {sim }}^{\sigma}\left(A_{3}, B\right)>\mathfrak{C}_{\text {sim }}^{\sigma}\left(A_{1}, B\right)>\mathfrak{C}_{\text {sim }}^{\sigma}\left(A_{2}, B\right)> \\
\quad \mathfrak{C}_{\text {sim }}^{\varpi}\left(A_{4}, B\right)>\mathfrak{C}_{\text {sim }}^{\varpi}\left(A_{5}, B\right)\end{array}$ \\
\hline & $K_{3}\left(A_{5}, B\right)=0.8351$ & $\mathfrak{C}_{\text {sim }}^{\sigma}\left(A_{5}, B\right)=0.8399$ & \\
\hline \multirow[t]{4}{*}{ Example 2 [57] } & $K_{2}\left(Q_{1}, P\right)=0.9412$ & $\mathfrak{C}_{s i m}\left(Q_{1}, P\right)=0.9813$ & $\begin{array}{r}K_{2}\left(Q_{1}, P\right)>K_{2}\left(Q_{3}, P\right)> \\
\left.K_{2}\left(Q_{2}, P\right)>K_{2}\left(Q_{4}, P\right)\right)\end{array}$ \\
\hline & $K_{2}\left(Q_{2}, P\right)=0.8109$ & $\mathfrak{C}_{\text {sim }}\left(Q_{2}, P\right)=0.8772$ & \\
\hline & $K_{2}\left(A_{3}, B\right)=0.8132$ & $\mathfrak{C}_{\text {sim }}\left(Q_{3}, P\right)=0.8487$ & $\begin{array}{l}\mathfrak{C}_{\text {sim }}\left(Q_{1}, P\right)>\mathfrak{C}_{\text {sim }}\left(Q_{2}, P\right)> \\
\quad \mathfrak{C}_{\text {sim }}\left(Q_{3}, P\right)>\mathfrak{C}_{\text {sim }}\left(Q_{4}, P\right)\end{array}$ \\
\hline & $K_{2}\left(A_{4}, B\right)=0.5923$ & $\mathfrak{C}_{s i m}\left(Q_{4}, P\right)=0.7040$ & \\
\hline \multirow[t]{4}{*}{$\begin{array}{l}\text { Example } 2[57] \varpi= \\
\quad(0.3,0.2,0.1,0.4)\end{array}$} & $K_{4}\left(Q_{1}, P\right)=0.9015$ & $\mathfrak{C}_{\text {sim }}^{\sigma}\left(Q_{1}, P\right)=0.9709$ & $\begin{array}{r}K_{4}\left(Q_{1}, P\right)>K_{4}\left(Q_{2}, P\right)> \\
\left.K_{4}\left(Q_{3}, P\right)>K_{4}\left(Q_{4}, P\right)\right)\end{array}$ \\
\hline & $K_{4}\left(Q_{2}, P\right)=0.8433$ & $\mathfrak{C}_{\text {sim }}^{\sigma}\left(Q_{2}, P\right)=0.8660$ & \\
\hline & $K_{4}\left(A_{3}, B\right)=0.7935$ & $\mathfrak{C}_{s i m}^{\varpi}\left(Q_{3}, P\right)=0.8066$ & $\begin{array}{l}\mathfrak{C}_{\text {sim }}^{\sigma}\left(Q_{1}, P\right)>\mathfrak{C}_{\text {sim }}^{\varpi}\left(Q_{2}, P\right)> \\
\quad \mathfrak{C}_{\text {sim }}^{\varpi}\left(Q_{3}, P\right)>\mathfrak{C}_{\text {sim }}^{\varpi}\left(Q_{4}, P\right)\end{array}$ \\
\hline & $K_{4}\left(A_{4}, B\right)=0.5969$ & $\mathfrak{C}_{\text {sim }}^{\pi}\left(Q_{4}, P\right)=0.7004$ & \\
\hline
\end{tabular}

Table 15 Comparison of CLDFS-based CDM (or WCDM) with some of existing CPyFS-based DMs

\begin{tabular}{|c|c|c|c|}
\hline Problem & $\begin{array}{l}\text { Result of the proposed } \\
\text { CPyFS-based DM }\end{array}$ & $\begin{array}{l}\text { Result of Our CDM (or } \\
\text { WCDM) }\end{array}$ & $\begin{array}{l}\text { Ranking for both DMs (CPyFS- } \\
\text { based DM and Our CDM) }\end{array}$ \\
\hline \multirow[t]{3}{*}{$\begin{array}{l}\text { Example } 1 \text { (Table 2) [60] } \\
a_{i}=b_{i}=c_{i}=0.3333 \varpi= \\
(0.11,0.14,0.1,0.18,0.21 \\
0.1,0.16)\end{array}$} & $W D_{C P y F S}^{1}\left(A_{1}, A\right)=0.5684$ & $\mathfrak{C}_{d i s}^{\sigma}\left(A_{1}, A\right)=0.8835$ & \multirow[t]{3}{*}{$\begin{array}{c}W D_{C P y F S}^{1}\left(A_{1}, A\right)>W D_{C P y F S}^{1}\left(A_{3}, A\right)> \\
W D_{C P y F S}^{1}\left(A_{2}, A\right)>W D_{C P y F S}^{1}\left(A_{4}, A\right)\end{array}$} \\
\hline & $W D_{C P y F S}^{1}\left(A_{3}, A\right)=0.5435$ & $\mathfrak{C}_{d i s}^{\sigma}\left(A_{3}, A\right)=0.7894$ & \\
\hline & $W D_{C P y F S}^{1}\left(A_{4}, A\right)=0.4227$ & $\mathfrak{C}_{d i s}^{\sigma}\left(A_{4}, A\right)=0.5942$ & \\
\hline \multirow{4}{*}{$\begin{array}{l}\text { Table } 6 \text { [60] } \\
a_{i}=b_{i}=c_{i}=0.3333 \\
\bar{\varpi}_{i}=\frac{1}{7}\end{array}$} & $W D_{C P y F S}^{1}\left(A_{1}, A\right)=0.5535$ & $\mathfrak{C}_{d i s}\left(A_{1}, A\right)=0.7124$ & \multirow{4}{*}{$\begin{array}{l}W D_{C P y F S}^{1}\left(A_{2}, A\right)>W D_{C P y F S}^{1}\left(A_{3}, A\right)> \\
W D_{C P y F S}^{1}\left(A_{4}, A\right)>W D_{C P y F S}^{1}\left(A_{1}, A\right) \\
\mathfrak{C}_{d i s}\left(A_{2}, A\right)>\mathfrak{C}_{d i s}\left(A_{3}, A\right)> \\
\quad \mathfrak{C}_{d i s}\left(A_{4}, A\right)>\mathfrak{C}_{d i s}^{\sigma}\left(A_{1}, A\right)\end{array}$} \\
\hline & $W D_{C P y F S}^{1}\left(A_{2}, A\right)=0.6087$ & $\mathfrak{C}_{d i s}\left(A_{2}, A\right)=0.915$ & \\
\hline & $W D_{C P y F S}^{1}\left(A_{3}, A\right)=0.5875$ & $\mathfrak{C}_{d i s}\left(A_{3}, A\right)=0.85$ & \\
\hline & $W D_{C P y F S}^{1}\left(A_{4}, A\right)=0.5716$ & $\mathfrak{C}_{\text {dis }}\left(A_{4}, A\right)=0.7389$ & \\
\hline
\end{tabular}


- Creating some new decision-making procedures based on the theory of information measure in handling reallife problems such as pattern recognition or cluster analysis.

3. Decision-making methods for CLDFS.

- Creating CLDFS-based algorithms to deal with decisionmaking problems such as strategic emerging industries evaluation, Internet stock investment, company investment decision, software development investment, movie recommender system, technologic innovation evaluation, and domestic airlines evaluation.

- Introducing new styles of decision-making technology to fill gaps in the CLDFS environment, such as interactive or dynamic multicriteria group decisionmaking.

In addition, the hybridization of CLDFS with hesitancy or bipolarity will be a hot topic in the near future.

\section{Declarations}

Conflict of interest The author declares that has no conflicts of interest regarding the publication of this paper.

Open Access This article is licensed under a Creative Commons Attribution 4.0 International License, which permits use, sharing, adaptation, distribution and reproduction in any medium or format, as long as you give appropriate credit to the original author(s) and the source, provide a link to the Creative Commons licence, and indicate if changes were made. The images or other third party material in this article are included in the article's Creative Commons licence, unless indicated otherwise in a credit line to the material. If material is not included in the article's Creative Commons licence and your intended use is not permitted by statutory regulation or exceeds the permitted use, you will need to obtain permission directly from the copyright holder. To view a copy of this licence, visit http://creativecomm ons.org/licenses/by/4.0/.

\section{Appendix}

Proof (for Proposition 3.13) Let us prove the parts (i) and (iii), and the others are similar.

(i) Let $\ell_{1}$ and $\ell_{2}$ be two CLDFNs, and then, we have 


$$
\begin{aligned}
\ell_{1} \oplus \ell_{2}= & \left(\begin{array}{l}
\left.\left(\Delta_{\mathfrak{L}}^{1}+\Delta_{\mathfrak{L}}^{2}-\Delta_{\mathfrak{L}}^{1} \Delta_{\mathfrak{L}}^{2}, \xi_{\Delta_{\mathfrak{L}}^{1}}+\xi_{\Delta_{\mathfrak{L}}^{2}}-\xi_{\Delta_{\mathfrak{L}}^{1}} \xi_{\Delta_{\mathfrak{L}}^{2}}\right),\left(\Phi_{\mathfrak{L}}^{1} \Phi_{\mathfrak{L}}^{2}, \xi_{\Phi_{\mathfrak{L}}^{1}} \xi_{\Phi_{\mathfrak{L}}^{2}}\right)\right), \\
\left.\left(\rho_{\mathfrak{L}}^{1}+\rho_{\mathfrak{L}}^{2}-\rho_{\mathfrak{L}}^{1} \rho_{\mathfrak{L}}^{2}, \xi_{\rho_{\mathfrak{L}}^{1}}+\xi_{\rho_{\mathfrak{L}}^{2}}-\xi_{\rho_{\mathfrak{L}}^{1}} \xi_{\rho_{\mathfrak{L}}^{2}}\right),\left(\sigma_{\mathfrak{L}}^{1} \sigma_{\mathfrak{L}}^{2}, \xi_{\sigma_{\mathfrak{L}}^{1}} \xi_{\sigma_{\mathfrak{L}}^{2}}\right)\right\rangle
\end{array}\right) \\
= & \left(\begin{array}{l}
\left\langle\left(\Delta_{\mathfrak{L}}^{2}+\Delta_{\mathfrak{L}}^{1}-\Delta_{\mathfrak{L}}^{2} \Delta_{\mathfrak{L}}^{1}, \xi_{\Delta_{\mathfrak{L}}^{2}}+\xi_{\Delta_{\mathfrak{L}}^{1}}-\xi_{\Delta_{\mathfrak{L}}^{2}} \xi_{\Delta_{\mathfrak{L}}^{1}}\right),\left(\Phi_{\mathfrak{L}}^{2} \Phi_{\mathfrak{L}}^{1}, \xi_{\Phi_{\mathfrak{L}}^{2}} \xi_{\Phi_{\mathfrak{L}}^{1}}\right)\right), \\
\left.\left(\rho_{\mathfrak{L}}^{2}+\rho_{\mathfrak{L}}^{1}-\rho_{\mathfrak{L}}^{2} \rho_{\mathfrak{L}}^{1}, \xi_{\rho_{\mathfrak{L}}^{2}}+\xi_{\rho_{\mathfrak{L}}^{1}}-\xi_{\rho_{\mathfrak{L}}^{2}} \xi_{\rho_{\mathfrak{L}}^{1}}\right),\left(\sigma_{\mathfrak{L}}^{2} \sigma_{\mathfrak{L}}^{1}, \xi_{\sigma_{\mathfrak{L}}^{2}} \xi_{\sigma_{\mathfrak{L}}^{1}}\right)\right\rangle
\end{array}\right) \\
= & \ell_{2} \oplus \ell_{1} .
\end{aligned}
$$

(iii) Let $\ell_{1}, \ell_{2}$ and $\ell_{3}$ be the CLDFNs, and then, we have

$$
\begin{aligned}
& \left(\ell_{1} \oplus \ell_{2}\right) \oplus \ell_{3}=\left(\begin{array}{l}
\left.\left(\Delta_{\mathfrak{L}}^{1}+\Delta_{\mathfrak{L}}^{2}-\Delta_{\mathfrak{L}}^{1} \Delta_{\mathfrak{L}}^{2}, \xi_{\Delta_{\mathfrak{L}}^{1}}+\xi_{\Delta_{\mathfrak{L}}^{2}}-\xi_{\Delta_{\mathfrak{L}}^{1}} \xi_{\Delta_{\mathfrak{L}}^{2}}\right),\left(\Phi_{\mathfrak{L}}^{1} \Phi_{\mathfrak{L}}^{2}, \xi_{\Phi_{\mathfrak{L}}^{1}} \xi_{\Phi_{\mathfrak{L}}^{2}}\right)\right\rangle, \\
\left.\left(\rho_{\mathfrak{L}}^{1}+\rho_{\mathfrak{L}}^{2}-\rho_{\mathfrak{L}}^{1} \rho_{\mathfrak{L}}^{2}, \xi_{\rho_{\mathfrak{L}}^{1}}+\xi_{\rho_{\mathfrak{L}}^{2}}-\xi_{\rho_{\mathfrak{L}}^{1}} \xi_{\rho_{\mathfrak{L}}^{2}}\right),\left(\sigma_{\mathfrak{L}}^{1} \sigma_{\mathfrak{L}}^{2}, \xi_{\sigma_{\mathfrak{L}}^{1}} \xi_{\sigma_{\mathfrak{L}}^{2}}\right)\right)^{2}
\end{array}\right) \oplus \ell_{3}
\end{aligned}
$$

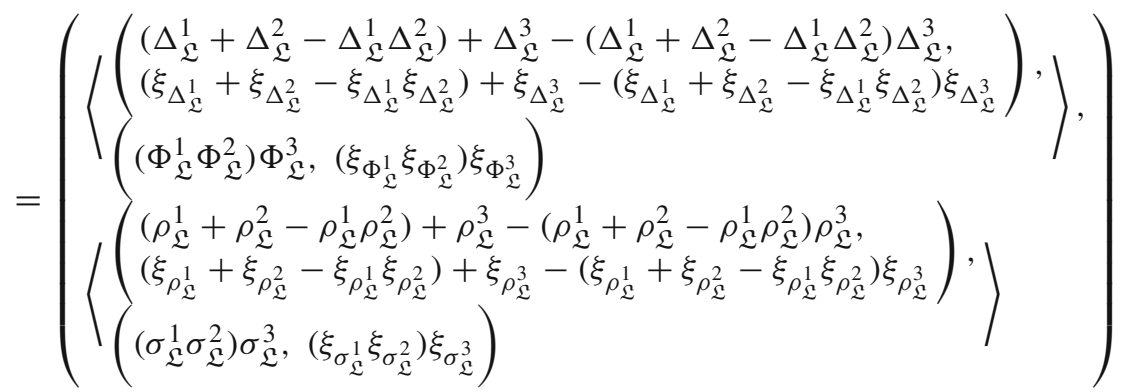

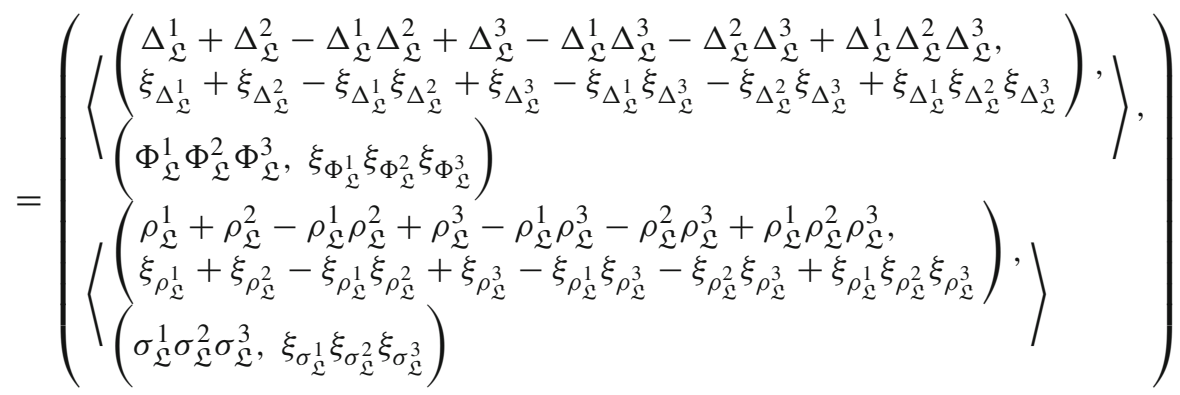

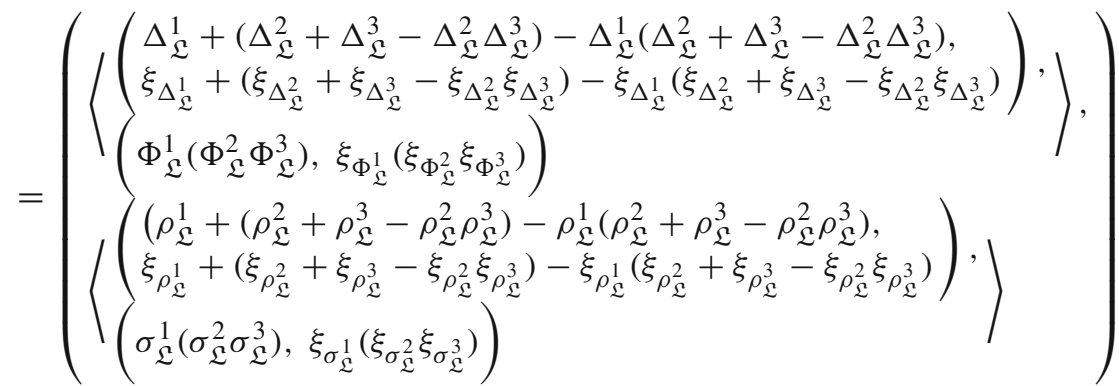$$
=\ell_{1} \oplus\left(\begin{array}{l}
\left.\left(\Delta_{\mathfrak{L}}^{2}+\Delta_{\mathfrak{L}}^{3}-\Delta_{\mathcal{L}}^{2} \Delta_{\mathfrak{L}}^{3}, \xi_{\Delta_{\mathfrak{L}}^{2}}+\xi_{\Delta_{\mathcal{L}}^{3}}-\xi_{\Delta_{\mathfrak{L}}^{2}} \xi_{\Delta_{\mathfrak{L}}^{3}}\right),\left(\Phi_{\mathfrak{L}}^{2} \Phi_{\mathfrak{L}}^{3}, \xi_{\Phi_{\mathfrak{L}}^{2}} \xi_{\Phi_{\mathfrak{L}}^{3}}\right)\right\rangle, \\
\left.\left(\rho_{\mathfrak{L}}^{2}+\rho_{\mathfrak{L}}^{3}-\rho_{\mathfrak{L}}^{2} \rho_{\mathfrak{L}}^{3}, \xi_{\rho_{\mathfrak{L}}^{2}}+\xi_{\rho_{\mathfrak{L}}^{3}}-\xi_{\rho_{\mathfrak{L}}^{2}} \xi_{\rho_{\mathfrak{L}}^{3}}\right),\left(\sigma_{\mathfrak{L}}^{2} \sigma_{\mathfrak{L}}^{3}, \xi_{\sigma_{\mathfrak{L}}^{2}} \xi_{\sigma_{\mathfrak{L}}^{3}}\right)\right\rangle
\end{array}\right)
$$

$=\ell_{1} \oplus\left(\ell_{2} \oplus \ell_{3}\right)$. 
Proof (for Proposition 3.14) Let us prove the part (ii), and the others are similar.

(ii) Let $\ell_{1}$ and $\ell_{2}$ be two CLDFNs and $\lambda>0$, and then

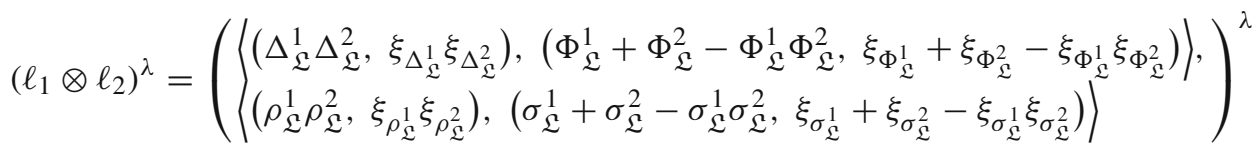

$$
\begin{aligned}
& =\left(\begin{array}{l}
\left\langle\left(\left(\Delta_{\mathfrak{L}}^{1} \Delta_{\mathfrak{L}}^{2}\right)^{\lambda},\left(\xi_{\Delta_{\mathfrak{L}}^{1}} \xi_{\Delta_{\mathfrak{L}}^{2}}\right)^{\lambda}\right),\left(1-\left(1-\left(\Phi_{\mathfrak{L}}^{1}+\Phi_{\mathfrak{L}}^{2}-\Phi_{\mathfrak{L}}^{1} \Phi_{\mathfrak{L}}^{2}\right)\right)^{\lambda}, 1-\left(1-\left(\xi_{\Phi_{\mathfrak{L}}^{1}}+\xi_{\Phi_{\mathfrak{L}}^{2}}-\xi_{\Phi_{\mathfrak{L}}^{1}} \xi_{\Phi_{\mathfrak{L}}^{2}}\right)\right)^{\lambda}\right)\right\rangle, \\
\left.\left.\left(\left(\rho_{\mathfrak{L}}^{1} \rho_{\mathfrak{L}}^{2}\right)^{\lambda},\left(\xi_{\rho_{\mathfrak{L}}^{1}} \xi_{\rho_{\mathfrak{L}}^{2}}\right)^{\lambda}\right),\left(1-\left(1-\left(\sigma_{\mathfrak{L}}^{1}+\sigma_{\mathfrak{L}}^{2}-\sigma_{\mathfrak{L}}^{1} \sigma_{\mathfrak{L}}^{2}\right)\right)^{\lambda}, 1-\left(1-\left(\xi_{\sigma_{\mathfrak{L}}^{1}}+\xi_{\sigma_{\mathfrak{L}}^{2}}-\xi_{\sigma_{\mathfrak{L}}^{1}} \xi_{\sigma_{\mathfrak{L}}^{2}}\right)\right)^{\lambda}\right)\right\rangle\right)
\end{array}\right)
\end{aligned}
$$

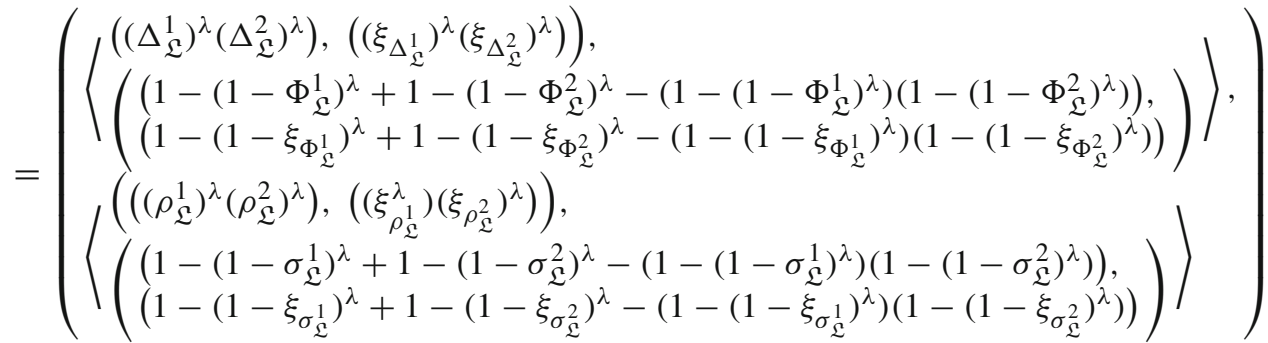

$$
\begin{aligned}
& =\ell_{1}^{\lambda} \otimes \ell_{2}^{\lambda} \text {. }
\end{aligned}
$$

Proof (for Properties of CSM in Eq. (4.2))

1. We consider $\mathfrak{C}_{\text {sim }}\left(\mathfrak{L}_{1}, \mathfrak{L}_{2}\right)=$

$$
\frac{1}{s} \sum_{k=1}^{s} \frac{\left(\begin{array}{l}
\Delta_{\mathfrak{L}_{1}}\left(r_{k}\right) \Delta_{\mathfrak{L}_{2}}\left(r_{k}\right)+\xi_{\Delta_{\mathfrak{L}_{1}}}\left(r_{k}\right) \xi_{\Delta_{\mathfrak{L}_{2}}}\left(r_{k}\right)+\Phi_{\mathfrak{L}_{1}}\left(r_{k}\right) \Phi_{\mathfrak{L}_{2}}\left(r_{k}\right)+ \\
\xi_{\Phi_{\mathfrak{L}_{1}}}\left(r_{k}\right) \xi_{\Phi_{\mathfrak{L}_{2}}}\left(r_{k}\right)+\rho_{\mathfrak{L}_{1}}^{k} \rho_{\mathfrak{L}_{2}}^{k}+\xi_{\left(\rho_{\mathfrak{L}_{1}}\right)} \xi_{\left(\rho_{\mathfrak{L}_{2}}\right)}+\sigma_{\mathfrak{L}_{1}} \sigma_{\mathfrak{L}_{2}}^{k}+\xi_{\left(\sigma_{\mathfrak{L}_{1}}\right)} \xi_{\left(\sigma_{\mathfrak{L}_{2}}\right)}
\end{array}\right)}{\left(\begin{array}{l}
\left.\left(\Delta_{\mathfrak{L}_{1}}\left(r_{k}\right)\right)^{2}+\left(\xi_{\Delta_{\mathfrak{L}_{1}}}\left(r_{k}\right)\right)^{2}+\left(\Phi_{\mathfrak{L}_{1}}\left(r_{k}\right)\right)^{2}+\left(\xi_{\Phi_{\mathfrak{L}_{1}}}\left(r_{k}\right)\right)^{2}+\left(\rho_{\mathfrak{L}_{1}}^{k}\right)^{2}+\left(\xi_{\left(\rho_{\mathfrak{L}_{1}}\right)}\right)^{2}+\left(\sigma_{\mathfrak{L}_{1}}^{k}\right)^{2}+\left(\xi_{\left(\sigma_{\mathfrak{L}_{1}}\right)}\right)^{2}\right)^{\frac{1}{2}} \times \\
\left(\left(\Delta_{\mathfrak{L}_{2}}\left(r_{k}\right)\right)^{2}+\left(\xi_{\Delta_{\mathfrak{L}_{2}}}\left(r_{k}\right)\right)^{2}+\left(\Phi_{\mathfrak{L}_{2}}\left(r_{k}\right)\right)^{2}+\left(\xi_{\Phi_{\mathfrak{L}_{2}}}\left(r_{k}\right)\right)^{2}+\left(\rho_{\mathfrak{L}_{2}}^{k}\right)^{2}+\left(\xi_{\left(\rho_{\mathfrak{L}_{2}}\right)}\right)^{2}+\left(\sigma_{\mathfrak{L}_{2}}^{k}\right)^{2}+\left(\xi_{\left(\sigma_{\mathfrak{L}_{2}}\right)}\right)^{2}\right)^{\frac{1}{2}}
\end{array}\right)} .
$$

Since all of the grades are in $[0,1], \mathfrak{C}_{\text {sim }}\left(\mathfrak{L}_{1}, \mathfrak{L}_{2}\right) \geq 0$.

Also, we have

$$
\begin{aligned}
& \left(\begin{array}{l}
\Delta_{\mathfrak{L}_{1}}\left(r_{k}\right) \Delta_{\mathfrak{L}_{2}}\left(r_{k}\right)+\xi_{\Delta_{\mathfrak{L}_{1}}}\left(r_{k}\right) \xi_{\Delta_{\mathfrak{L}_{2}}}\left(r_{k}\right)+\Phi_{\mathfrak{L}_{1}}\left(r_{k}\right) \Phi_{\mathfrak{L}_{2}}\left(r_{k}\right)+ \\
\xi_{\Phi_{\mathfrak{L}_{1}}}\left(r_{k}\right) \xi_{\Phi_{\mathfrak{L}_{2}}}\left(r_{k}\right)+\rho_{\mathfrak{L}_{1}}^{k} \rho_{\mathfrak{L}_{2}}^{k}+\xi_{\left(\rho_{\mathfrak{L}_{1}}\right.}^{k} \xi_{\left(\rho_{\mathfrak{L}_{2}}\right)}^{k}+\sigma_{\mathfrak{L}_{1}}^{k} \sigma_{\mathfrak{L}_{2}}^{k}+\xi_{\left(\sigma_{\mathfrak{L}_{1}}\right)} \xi_{\left(\sigma_{\mathfrak{L}_{2}}^{k}\right)}^{k}
\end{array}\right) \\
& \quad \leq\left(\begin{array}{l}
\left(\left(\Delta_{\mathfrak{L}_{1}}\left(r_{k}\right)\right)^{2}+\left(\xi_{\Delta_{\mathfrak{L}_{1}}}\left(r_{k}\right)\right)^{2}+\left(\Phi_{\mathfrak{L}_{1}}\left(r_{k}\right)\right)^{2}+\left(\xi_{\Phi_{\mathfrak{L}_{1}}}\left(r_{k}\right)\right)^{2}+\left(\rho_{\mathfrak{L}_{1}}^{k}\right)^{2}+\left(\xi_{\left(\rho_{\mathfrak{L}_{1}}\right)}\right)^{2}+\left(\sigma_{\mathfrak{L}_{1}}^{k}\right)^{2}+\left(\xi_{\left(\sigma_{\mathfrak{L}_{1}}\right)}\right)^{2}\right)^{\frac{1}{2}} \times \\
\left.\left(\Delta_{\mathfrak{L}_{2}}\left(r_{k}\right)\right)^{2}+\left(\xi_{\Delta_{\mathfrak{L}_{2}}}\left(r_{k}\right)\right)^{2}+\left(\Phi_{\mathfrak{L}_{2}}\left(r_{k}\right)\right)^{2}+\left(\xi_{\Phi_{\mathfrak{L}_{2}}}\left(r_{k}\right)\right)^{2}+\left(\rho_{\mathfrak{L}_{2}}^{k}\right)^{2}+\left(\xi_{\left(\rho_{\mathfrak{L}_{2}}\right)}\right)^{2}+\left(\sigma_{\mathfrak{L}_{2}}^{k}\right)^{2}+\left(\xi_{\left(\sigma_{\mathfrak{L}_{2}}\right.}\right)^{2}\right)^{\frac{1}{2}}
\end{array}\right)
\end{aligned}
$$

using Hölder's inequality: for all $\left(a_{1}, a_{2}, \ldots, a_{s}\right),\left(b_{1}, b_{2}, \ldots, b_{s}\right) \in$

$\mathbb{R}^{s}$ (where $s$ is a positive integer and $\mathbb{R}^{s}$ denotes $s$ -

dimensional Euclidean space)

$$
\sum_{k=1}^{s}\left|a_{k} b_{k}\right| \leq\left(\sum_{k=1}^{s}\left|a_{k}\right|^{p}\right)^{\frac{1}{p}}\left(\sum_{k=1}^{s}\left|b_{k}\right|^{q}\right)^{\frac{1}{q}}
$$

with $p$ and $q$ satisfy the condition $\frac{1}{p}+\frac{1}{q}=1$. Therefore, $\mathfrak{C}_{\text {sim }}\left(\mathfrak{L}_{1}, \mathfrak{L}_{2}\right) \leq 1$. Thus, the assertion is confirmed.

2. This assertion can be easily verified, so it is omitted. 
3. $\Rightarrow:$ If $\mathfrak{L}_{1}=\mathfrak{L}_{2}$, then we have

$$
\begin{aligned}
& \mathfrak{C}_{\text {sim }}\left(\mathfrak{L}_{1}, \mathfrak{L}_{1}\right)
\end{aligned}
$$

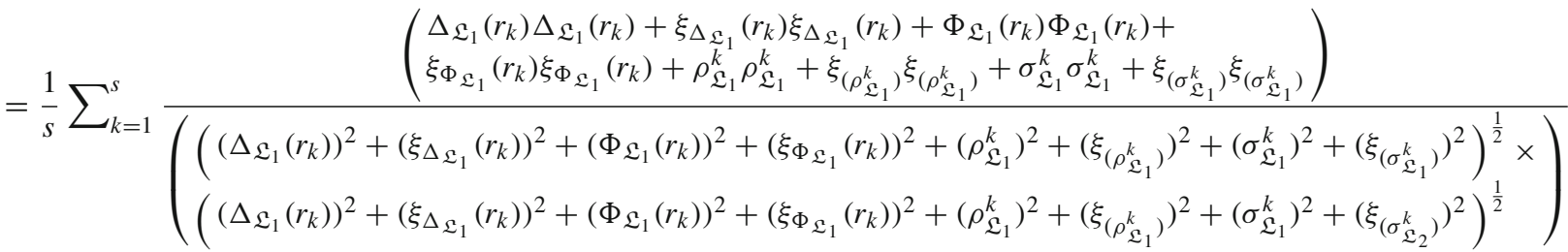

$$
\begin{aligned}
& =\frac{1}{s} \sum_{k=1}^{s} \frac{\left(\Delta_{\mathcal{L}_{1}}\left(r_{k}\right)\right)^{2}+\left(\xi_{\Delta \mathfrak{L}_{1}}\left(r_{k}\right)\right)^{2}+\left(\Phi_{\mathfrak{L}_{1}}\left(r_{k}\right)\right)^{2}+\left(\xi_{\Phi_{\mathfrak{L}_{1}}}\left(r_{k}\right)\right)^{2}+\left(\rho_{\mathcal{L}_{1}}^{k}\right)^{2}+\left(\xi_{\left(\rho_{\mathcal{L}_{1}}^{k}\right)}\right)^{2}+\left(\sigma_{\mathfrak{L}_{1}}^{k}\right)^{2}+\left(\xi_{\left(\sigma_{\mathfrak{L}_{1}}^{k}\right.}\right)^{2}}{\left(\Delta_{\mathcal{L}_{1}}\left(r_{k}\right)\right)^{2}+\left(\xi_{\Delta_{\mathfrak{L}_{1}}}\left(r_{k}\right)\right)^{2}+\left(\Phi_{\mathcal{L}_{1}}\left(r_{k}\right)\right)^{2}+\left(\xi_{\Phi_{\mathfrak{L}_{1}}}\left(r_{k}\right)\right)^{2}+\left(\rho_{\mathfrak{L}_{1}}^{k}\right)^{2}+\left(\xi_{\left(\rho_{\mathfrak{L}_{1}}^{k}\right)}\right)^{2}+\left(\sigma_{\mathfrak{L}_{1}}^{k}\right)^{2}+\left(\xi_{\left(\sigma_{\mathfrak{L}_{1}}\right.}^{k}\right)^{2}} \\
& =1
\end{aligned}
$$

$\Leftarrow:$ If $\mathfrak{C}_{\text {sim }}\left(\mathfrak{L}_{1}, \mathfrak{L}_{2}\right)=1$, that is

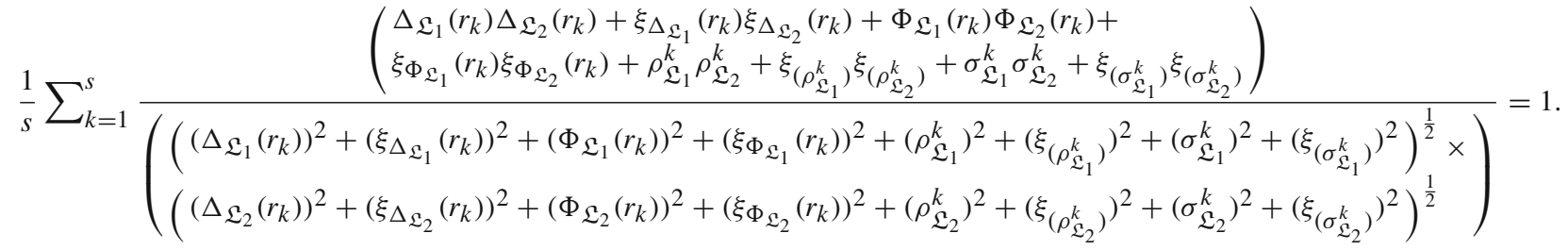

Then, we can write

$$
\sum_{k=1}^{s} \frac{\left(\begin{array}{l}
\Delta_{\mathfrak{L}_{1}}\left(r_{k}\right) \Delta_{\mathfrak{L}_{2}}\left(r_{k}\right)+\xi_{\Delta_{\mathfrak{L}_{1}}}\left(r_{k}\right) \xi_{\Delta_{\mathfrak{L}_{2}}}\left(r_{k}\right)+\Phi_{\mathfrak{L}_{1}}\left(r_{k}\right) \Phi_{\mathfrak{L}_{2}}\left(r_{k}\right)+ \\
\xi_{\Phi_{\mathfrak{L}_{1}}}\left(r_{k}\right) \xi_{\Phi_{\mathcal{L}_{2}}}\left(r_{k}\right)+\rho_{\mathfrak{L}_{1}}^{k} \rho_{\mathfrak{L}_{2}}^{k}+\xi_{\left(\rho_{\mathfrak{L}_{1}}^{k}\right)} \xi_{\left(\rho_{\mathfrak{L}_{2}}^{k}\right)}+\sigma_{\mathfrak{L}_{1}}^{k} \sigma_{\mathfrak{L}_{2}}^{k}+\xi_{\left(\sigma_{\mathfrak{L}_{1}}^{k}\right.} \xi_{\left(\sigma_{\mathfrak{L}_{2}}^{k}\right)}
\end{array}\right)}{\left(\begin{array}{l}
\left.\left(\Delta_{\mathfrak{L}_{1}}\left(r_{k}\right)\right)^{2}+\left(\xi_{\Delta_{\mathfrak{L}_{1}}}\left(r_{k}\right)\right)^{2}+\left(\Phi_{\mathfrak{L}_{1}}\left(r_{k}\right)\right)^{2}+\left(\xi_{\Phi_{\mathfrak{L}_{1}}}\left(r_{k}\right)\right)^{2}+\left(\rho_{\mathfrak{L}_{1}}^{k}\right)^{2}+\left(\xi_{\left(\rho_{\mathfrak{L}_{1}}^{k}\right)}\right)^{2}+\left(\sigma_{\mathfrak{L}_{1}}^{k}\right)^{2}+\left(\xi_{\left(\sigma_{\mathfrak{L}_{1}}^{k}\right.}\right)^{2}\right)^{\frac{1}{2}} \times \\
\left(\left(\Delta_{\mathfrak{L}_{2}}\left(r_{k}\right)\right)^{2}+\left(\xi_{\Delta_{\mathfrak{L}_{2}}}\left(r_{k}\right)\right)^{2}+\left(\Phi_{\mathfrak{L}_{2}}\left(r_{k}\right)\right)^{2}+\left(\xi_{\Phi_{\mathfrak{L}_{2}}}\left(r_{k}\right)\right)^{2}+\left(\rho_{\mathfrak{L}_{2}}^{k}\right)^{2}+\left(\xi_{\left(\rho_{\mathfrak{L}_{2}}^{k}\right)}\right)^{2}+\left(\sigma_{\mathfrak{L}_{2}}^{k}\right)^{2}+\left(\xi_{\left(\sigma_{\mathfrak{L}_{2}}^{k}\right)}\right)^{2}\right)^{\frac{1}{2}}
\end{array}\right)}
$$

By considering Eq. (7.2), we get from Eq. (7.3) that for all $k=1,2, \ldots, s$

$$
\begin{aligned}
& \left(\begin{array}{l}
\Delta_{\mathfrak{L}_{1}}\left(r_{k}\right) \Delta_{\mathfrak{L}_{2}}\left(r_{k}\right)+\xi_{\Delta_{\mathfrak{L}_{1}}}\left(r_{k}\right) \xi_{\Delta_{\mathfrak{L}_{2}}}\left(r_{k}\right)+\Phi_{\mathfrak{L}_{1}}\left(r_{k}\right) \Phi_{\mathfrak{L}_{2}}\left(r_{k}\right)+ \\
\xi_{\Phi_{\mathfrak{L}_{1}}}\left(r_{k}\right) \xi_{\Phi_{\mathfrak{L}_{2}}}\left(r_{k}\right)+\rho_{\mathfrak{L}_{1}}^{k} \rho_{\mathfrak{L}_{2}}^{k}+\xi_{\left(\rho_{\mathfrak{L}_{1}}^{k}\right.} \xi_{\left(\rho_{\left.\mathcal{L}_{2}\right)}^{k}\right.}+\sigma_{\mathfrak{L}_{1}}^{k} \sigma_{\mathfrak{L}_{2}}^{k}+\xi_{\left(\sigma_{\mathfrak{L}_{1}}^{k}\right)} \xi_{\left(\sigma_{\mathfrak{L}_{2}}^{k}\right)}
\end{array}\right)
\end{aligned}
$$

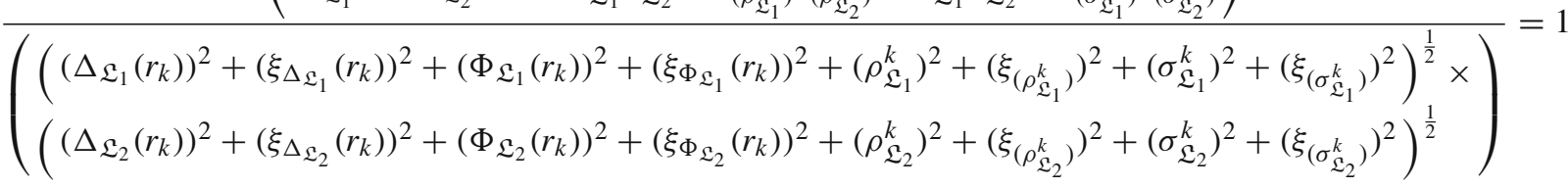

and so

$$
\begin{aligned}
& \left(\begin{array}{l}
\Delta_{\mathfrak{L}_{1}}\left(r_{k}\right) \Delta_{\mathfrak{L}_{2}}\left(r_{k}\right)+\xi_{\Delta_{\mathfrak{L}_{1}}}\left(r_{k}\right) \xi_{\Delta_{\mathfrak{L}_{2}}}\left(r_{k}\right)+\Phi_{\mathfrak{L}_{1}}\left(r_{k}\right) \Phi_{\mathfrak{L}_{2}}\left(r_{k}\right)+ \\
\xi_{\Phi_{\mathfrak{L}_{1}}}\left(r_{k}\right) \xi_{\Phi_{\mathfrak{L}_{2}}}\left(r_{k}\right)+\rho_{\mathfrak{L}_{1}}^{k} \rho_{\mathfrak{L}_{2}}^{k}+\xi_{\left(\rho_{\mathfrak{L}_{1}}^{k}\right)} \xi_{\left(\rho_{\mathfrak{L}_{2}}^{k}\right)}+\sigma_{\mathfrak{L}_{1}}^{k} \sigma_{\mathfrak{L}_{2}}^{k}+\xi_{\left(\sigma_{\mathfrak{L}_{1}}\right)} \xi_{\left(\sigma_{\mathfrak{L}_{2}}^{k}\right)}^{k}
\end{array}\right) \\
& =\left(\begin{array}{l}
\left(\left(\Delta_{\mathfrak{L}_{1}}\left(r_{k}\right)\right)^{2}+\left(\xi_{\Delta_{\mathfrak{L}_{1}}}\left(r_{k}\right)\right)^{2}+\left(\Phi_{\mathfrak{L}_{1}}\left(r_{k}\right)\right)^{2}+\left(\xi_{\Phi_{\mathfrak{L}_{1}}}\left(r_{k}\right)\right)^{2}+\left(\rho_{\mathfrak{L}_{1}}^{k}\right)^{2}+\left(\xi_{\left(\rho_{\mathfrak{L}_{1}}^{k}\right)}\right)^{2}+\left(\sigma_{\mathfrak{L}_{1}}^{k}\right)^{2}+\left(\xi_{\left(\sigma_{\mathfrak{L}_{1}}^{k}\right)}\right)^{2}\right)^{\frac{1}{2}} \times \\
\left(\left(\Delta_{\mathfrak{L}_{2}}\left(r_{k}\right)\right)^{2}+\left(\xi_{\Delta_{\mathfrak{L}_{2}}}\left(r_{k}\right)\right)^{2}+\left(\Phi_{\mathfrak{L}_{2}}\left(r_{k}\right)\right)^{2}+\left(\xi_{\Phi_{\mathfrak{L}_{2}}}\left(r_{k}\right)\right)^{2}+\left(\rho_{\mathfrak{L}_{2}}^{k}\right)^{2}+\left(\xi_{\left(\rho_{\mathfrak{L}_{2}}^{k}\right)}\right)^{2}+\left(\sigma_{\mathfrak{L}_{2}}^{k}\right)^{2}+\left(\xi_{\left(\sigma_{\mathfrak{S}_{2}}\right)}\right)^{2}\right)^{\frac{1}{2}}
\end{array}\right) .
\end{aligned}
$$

This is possible provided that $\mathfrak{L}_{1}=\mathfrak{L}_{2}$. Thus, the proof is completed. 


\section{References}

1. Zadeh LA (1965) Fuzzy sets. Inf Control 8(3):338-353

2. Karaaslan F, Özlü Ş (2020) Correlation coefficients of dual type-2 hesitant fuzzy sets and their applications in clustering analysis. Int J Intell Syst 35(7):1200-1229

3. Riaz M, Tehrim ST (2020) A robust extension of VIKOR method for bipolar fuzzy sets using connection numbers of SPA theory based metric spaces. Artif Intell Rev (in press). https://doi.org/10. 1007/s10462-020-09859-w

4. Riaz M, Davvaz B, Fakhar A, Firdous A (2020) Hesitant fuzzy soft topology and its applications to multi-attribute group decisionmaking. Soft Comput (in press). https://doi.org/10.1007/s00500020-04938-0

5. Torra V (2010) Hesitant fuzzy sets. Int J Intell Syst 25:529-539

6. Atanassov KT (1986) Intuitionistic fuzzy sets. Fuzzy Set Syst 20:87-96

7. Ali MI, Feng F, Mahmood T, Mahmood I, Faizan H (2019) A graphical method for ranking Atanassov's intuitionistic fuzzy values using uncertainty index and entropy. Int J Intell Syst 34:2692-2712

8. Hayat K, Ali MI, Cao B-Y, Karaaslan F, Yang X-P (2018) Another view of group-based generalized intuitionistic fuzzy soft sets: aggregation operators and multiattribute decision making. Symmetry 10(12):253

9. Kumar K, Garg H (2018) Connection number of set pair analysis based TOPSIS method on intuitionistic fuzzy sets and their application to decision making. Appl Intell 48(8):2112-2119

10. Song Y, Wang X, Lei L, Xue A (2014) A new similarity measure between intuitionistic fuzzy sets and its application to pattern recognition. Abstr Appl Anal 2014(ArticleID 384241):11 pages

11. Uluçay V, Deli I, Şahin M (2019) Intuitionistic trapezoidal fuzzy multi-numbers and its application to multi-criteria decision-making problems. Complex Intell Syst 5:65-78

12. Vlachos IK, Sergiadis GD (2007) Intuitionistic fuzzy informationapplication to pattern recognition. Pattern Recognit Lett 28:197206

13. Ye J (2011) Cosine similarity measures for intuitionistic fuzzy sets and their applications. Math Comput Model 53:91-97

14. Yager RP (2013) Pythagorean fuzzy subsets. In: Proceedings of the IFSA World Congress and NAFIPS Annual Meeting, Edmonton, pp 57-61

15. Garg H (2017) Confidence levels based Pythagorean fuzzy aggregation operators and its application to decision-making process. Comput Math Org Theory 23(4):546-571

16. Hashmi MR, Riaz M (2020) A novel approach to censuses process by using Pythagorean m-polar fuzzy Dombi's aggregation operators. J Intell Fuzzy Syst 38(2):1977-1995

17. Peng X, Selvachandran G (2019) Pythagorean fuzzy set: state of the art and future directions. Artif Intell Rev 52:1873-1927

18. Nguyen XT, Nguyen VD, Nguyen VH, Garg H (2019) Exponential similarity measures for Pythagorean fuzzy sets and their applications to pattern recognition and decision-making process. Complex Intell Syst 5(2):217-228

19. Peng X, Garg H (2019) Multiparametric similarity measures on Pythagorean fuzzy sets with applications to pattern recognition. Appl Intell 49:4058-4096

20. Wei G, Wei Y (2018) Similarity measures of Pythagorean fuzzy sets based on the cosine function and their applications. Int J Intell Syst 33:634-652

21. Zhang Q, Hu J, Feng J, Liu A, Li Y (2019) New similarity measures of Pythagorean fuzzy sets and their applications. IEEE Access 7(3):138192-138202

22. Abdullah L, Goh P (2019) Decision making method based on Pythagorean fuzzy sets and its application to solid waste management. Complex Intell Syst 5:185-198
23. Akram M, Ilyas F, Garg H (2020) Multi-criteria group decision making based on ELECTRE-I method on Pythagorean fuzzy information. Soft Comput 24(5):3425-3453

24. Khalid N, Riaz M, Peng X, Afzal D (2019) Pythagorean fuzzy soft MCGDM methods based on TOPSIS, VIKOR and aggregation operators. J Intell Fuzzy Syst 37:6937-6957

25. Riaz M, Hashmi MR (2020) Soft rough Pythagorean m-polar fuzzy sets and Pythagorean m-polar fuzzy soft rough sets with application to decision-making. Comput Appl Math 39:16

26. Yager RP (2017) Generalized orthopair fuzzy sets. IEEE Trans Fuzzy Syst 25:1222-1230

27. Ali M (2018) Another view on q-rung orthopair fuzzy sets. Int J Intell Syst 33:2139-2153

28. Liu P, Wang P (2018) Some q-rung orthopair fuzzy aggregation operators and their applications to multiple-attribute decision making. Int J Intell Syst 33:259-280

29. Wang P, Wang J, Wei G, Wei C (2019) Similarity measures of q-rung orthopair fuzzy sets based on cosine function and their application. Mathematics 7(340):23

30. Zhang B, Mahmood T, Ahmmad J, Khan Q, Ali Z, Zeng S (2020) Cubic q-rung orthopair fuzzy Heronian mean operators and their applications to multi-attribute group decision making. Mathematics $8: 1125$

31. Cuong BC (2014) Picture fuzzy set. J Comput Sci Cybern 30:409_ 420

32. Hashmi MR, Riaz M, Smarandache F (2020) m-polar neutrosophic topology with applications to multi-criteria decision-making in medical diagnosis and clustering analysis. Int J Fuzzy Syst 22:273292

33. Mahmood T, Ullah K, Khan Q, Jan N (2019) An approach toward decision-making and medical diagnosis problemsusing the concept of spherical fuzzy sets. Neural Comput Appl 31:7041-7053

34. Ullah K, Garg H, Mahmood T, Jan N, Ali Z (2020) Correlation coefficients for T-spherical fuzzy sets and their applications in clustering and multi-attribute decision making. Soft Comput 24:1647-1659

35. Ullah K, Hassan N, Mahmood T, Jan N, Hassan M (2019) Evaluation of investment policy based on multi-attribute decision making using interval valued T-spherical fuzzy aggregation operators. Symmetry 11:357

36. Wang H, Smarandache F, Zhang YQ, Sunderraman R (2010) Single valued neutrosophic set. Multispace Multistruct 4:410-413

37. Molodtsov D (1999) Soft set theory-first results. Comput Math Appl 37:19-31

38. Çag̃man N, Karataş S (2013) Intuitionistic fuzzy soft set theory and its decision making. J Intell Fuzzy Syst 24(4):829-836

39. Kamaci H (2019) Interval-valued fuzzy parameterized intuitionistic fuzzy soft sets and their applications. Cumhuriyet Sci J 40(2):317-331

40. Karaaslan F (2016) Intuitionistic fuzzy parameterized intuitionistic fuzzy soft sets with applications in decision making. Ann Fuzzy Math Inf 11(4):607-619

41. Karaaslan F, Karataş S (2016) OR and AND-products of ifpintuitionistic fuzzy soft sets and their applications in decision making. J Intell Fuzzy Syst 31(3):1427-1434

42. Khan MJ, Kumam P, Liu P, Kumam W, Ashraf S (2019) A novel approach to generalized intuitionistic fuzzy soft sets and its application in decision support system. Mathematics 7(8):742

43. Bora M, Bora B, Neog TJ, Sut DK (2014) Intuitionistic fuzzy soft matrix theory and its application in medical diagnosis. Ann Fuzzy Math Inf 7(1):143-153

44. Guleria A, Bajaj RK (2019) On Pythagorean fuzzy soft matrices, operations and their applications in decision making and medical diagnosis. Soft Comput 23:7889-7900 
45. Kamacı H, Atagün AO, Toktaş E (2018) Bijective soft matrix theory and multi-bijective linguistic soft decision system. Filomat 32(11):3799-3814

46. Petchimuthu S, Kamac1 H (2019) The row-products of inverse soft matrices in multicriteria decision making. J Intell Fuzzy Syst 36(6):6425-6441

47. Aygün E, Kamacı H (2019) Some generalized operations in soft set theory and their role in similarity and decision making. J Intell Fuzzy Syst 36(6):6537-6547

48. Kamac1 H (2019) Similarity measure for soft matrices and its applications. J Intell Fuzzy Syst 36(4):3061-3072

49. Kumar T, Bajaj RK (2014) On complex intuitionistic fuzzy soft sets with distance measures and entropies. J Math 2014(Article ID 972198): 12

50. Petchimuthu S, Garg H, Kamacı H, Atagün AO (2020) The mean operators and generalized products of fuzzy soft matrices and their applications in MCGDM. Comput Appl Math 39(2):68

51. Ramot D, Milo R, Friedman MK (2002) Complex fuzzy sets. IEEE Trans Fuzzy Syst 10(2):171-186

52. Ramot D, Friedman M, Langholz G, Kandel A (2003) Complex fuzzy logic. IEEE Trans Fuzzy Syst 11(4):450-461

53. Ma X, Zhan J, Khan M, Zeeshan M, Anis S, Awan AS (2019) Complex fuzzy sets with applications in signals. Comput Appl Math 38(4): 150

54. Alkouri A, Salleh A (2012) Complex intuitionistic fuzzy sets. In: Ch. 2nd International Conference on Fundamental and Applied Sciences, pp 464-470

55. Alkouri A, Salleh A (2013) Complex Atanassov's intuitionistic fuzzy relation. Abstr Appl Anal 2013(Article ID 287382):18

56. Garg H, Rani D (2019) Exponential, logarithmic and compensative generalized aggregation operators under complex intuitionistic fuzzy environment. Group Decis Negot 28(5):991-1050

57. Garg H, Rani D (2019) A robust correlation coefficient measure of complex intuitionistic fuzzy sets and their applications in decisionmaking. Appl Intell 49:496-512

58. Rani D, Garg H (2018) Complex intuitionistic fuzzy power aggregation operators and their applications in multicriteria decisionmaking. Expert Syst 35(e12325):1-22

59. Rani D, Garg H (2017) Distance measures between the complex intuitionistic fuzzy sets and their applications to the decisionmaking process. Int J Uncertain Quan 7:423-439
60. Ullah K, Mahmood T, Ali Z, Jan N (2020) On some distance measures of complex Pythagorean fuzzy sets and their applications in pattern recognition. Complex Intell Syst 6:15-27

61. Liu P, Mahmood T, Ali Z (2020) Complex q-rung orthopair fuzzy aggregation operators and their applications in multiple-attribute group decision making. Information 11(5):28

62. Ali Z, Mahmood T (2020) Maclaurin symmetric mean operators and their applications in the environment of complex q-rung orthopair fuzzy sets. Comput Appl Math 39:161

63. Garg H, Gwak J, Mahmood T, Ali Z (2020) Power aggregation operators and VIKOR methods for complex q-rung orthopair fuzzy sets and their applications. Mathematics 8:538

64. Liu P, Ali Z, Mahmood T (2019) A method to multi-attribute group decision making problem with complex q-rung orthopair linguistic information based on Heronian mean operators. Int J Comput Intell Syst 12:1465-1496

65. Riaz M, Hashmi MR (2019) Linear diophantine fuzzy set and its applications towards multi-attribute decision making problems. J Intell Fuzzy Syst 37(4):5417-5439

66. Bhattacharya A (1946) On a measure of divergence of two multinomial populations. Sankhya 7:401-406

67. Salton G, McGill MJ (1983) Introduction to modern information retrieval. McGraw-Hill Book Company, New York

Publisher's Note Springer Nature remains neutral with regard to jurisdictional claims in published maps and institutional affiliations. 